\title{
Article
}

\section{Taxonomy of protoplanetary discs observed with ALMA}

Parker, Raeesa, Ward-Thompson, Derek and Kirk, Jason Matthew

Available at http://clok.uclan.ac.uk/40641/

Parker, Raeesa, Ward-Thompson, Derek ORCID: 0000-0003-1140-2761 and Kirk, Jason Matthew ORCID: 0000-0002-4552-7477 (2022) Taxonomy of protoplanetary discs observed with ALMA. Monthly Notices of the Royal Astronomical Society, 511 (2). pp. 2453-2490. ISSN 0035-8711

It is advisable to refer to the publisher's version if you intend to cite from the work. http://dx.doi.org/10.1093/mnras/stac152

For more information about UCLan's research in this area go to http://www.uclan.ac.uk/researchgroups/ and search for < name of research Group>.

For information about Research generally at UCLan please go to http://www.uclan.ac.uk/research/

All outputs in CLoK are protected by Intellectual Property Rights law, including Copyright law. Copyright, IPR and Moral Rights for the works on this site are retained by the individual authors and/or other copyright owners. Terms and conditions for use of this material are defined in the policies page.

\section{CLoK}

Central Lancashire online Knowledge www.clok.uclan.ac.uk

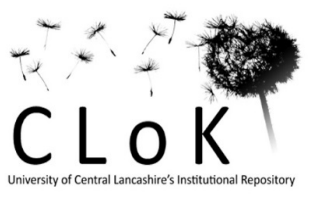




\title{
Taxonomy of protoplanetary discs observed with ALMA
}

\author{
Raeesa Parker, ${ }^{\star}$ Derek Ward-Thompson ${ }^{\circledR}$ and Jason Kirk \\ Jeremiah Horrocks Institute, University of Central Lancashire, Preston PR1 2HE, UK
}

Accepted 2022 January 12. Received 2022 January 5; in original form 2021 June 29

\begin{abstract}
Many observations of protoplanetary discs studied with ALMA have revealed the complex substructure present in the discs. Rings and gaps in the dust continuum are now a common sight in many discs; however, their origins still remain unknown. We look at all protoplanetary disc images taken with ALMA from cycles 0 to 5 and find that 56 discs show clear substructure. We further study the 56 discs and classify the morphology seen according to four categories: Rim, Ring, Horseshoe, and Spiral. We calculate the ages of the host stars using stellar isochrones and investigate the relation between the morphology of the substructure seen in the protoplanetary discs and the age of the host stars. We find that there is no clear evolutionary sequence in the protoplanetary discs as the stars increase in age, although there is a slight tendency for spirals to appear in younger systems and horseshoes to be seen in more evolved systems. We also show that majority of the images of protoplanetary discs made by ALMA may not have had a sufficiently high resolution or sensitivity to resolve substructure in the disc. We show that angular resolution is important in detecting substructure within protoplanetary discs, with sensitivity distinguishing between the different types of substructure. We compare the substructure seen in protoplanetary discs at sub-mm to those seen in scattered light. We find that cavities are a common substructure seen in discs at both sub-mm wavelengths and in scattered light.
\end{abstract}

Key words: protoplanetary discs - stars: pre-main-sequence - stars: protostars.

\section{INTRODUCTION}

ALMA observations of protoplanetary discs have revealed a multitude of different complex substructures. These include horseshoelike structures (Casassus et al. 2013), spiral arms (Pérez et al. 2016), inner cavities (van der Marel et al. 2015), and discs featuring bright, concentric dust rings (ALMA Partnership et al. 2015). There has also been an increase in the amount of theoretical work being undertaken in order to explain the origin of these substructures (Dong, Zhu \& Whitney 2015a; Takahashi \& Inutsuka 2016; Gonzalez, Laibe \& Maddison 2017).

A common substructure seen in the protoplanetary discs observed with ALMA is a cavity of some sort. This could be in the form of the inner cavities seen in transition discs or the depletion of dust and gas in different regions of a protoplanetary disc. These cavities may be caused by photoevaporation (Hollenbach et al. 1994; Hardy et al. 2015), planet-disc interactions (Lin \& Papaloizou 1979; Kley \& Nelson 2012; Baruteau et al. 2014; Dong et al. 2015a), or gravitational instabilities (Lorén-Aguilar \& Bate 2016; Takahashi \& Inutsuka 2016). Comparisons between cavities seen in the dust and the gas may be used to determine if planets in the disc are responsible for the depletions (van der Marel et al. 2016a; Boehler et al. 2017, 2018; Dong et al. 2017; van der Marel, Williams \& Bruderer 2018). Scattered light observations comparing the sizes of small and large grains may also be used to determine if planets are the origin of the inner cavities (de Juan Ovelar et al. 2013; Villenave et al. 2019).

^E-mail: rparker4@uclan.ac.uk
Dead zones may also be responsible for the inner cavities seen in transition discs (Pinilla et al. 2016).

Spiral density waves can also be seen in some protoplanetary discs at submillimetre wavelengths (Pérez et al. 2016). These structures are thought to be excited by either planet-disc interactions (Dong et al. 2015a; Zhu et al. 2015), gravitational instabilities (Dong et al. 2015b), or a combination of both (Pohl et al. 2015).

The interaction of a planet with a disc is commonly thought to be the origin mechanism for forming ring-like structures seen in protoplanetary discs. Fedele et al. (2018) were able to show that multiple gaps in a disc may be formed by single or multiple planets. Additional methods to explain the formation of dust rings in protoplanetary discs have also been theorized. Fast pebble growth near condensation fronts (Zhang, Blake \& Bergin 2015), secular gravitational instability (Takahashi \& Inutsuka 2016), and aggregate sintering (Okuzumi et al. 2016) are just some of the mechanisms proposed.

Submillimetre observations of the discs surrounding HD142527 and IRS48 have revealed azimuthal dust concentrations in the disc (Casassus et al. 2013; van der Marel et al. 2013). It is thought that these pile-ups of large dust grains are formed due to a pressure maximum at the edge of a gap in the disc. This pressure maximum can develop in a number of ways including the gas disc becoming unstable due to the Rossby Wave Instability (Baruteau \& Zhu 2016) or due to a binary companion (Ragusa et al. 2017). Embedded young protoplanets could also cause a pressure maximum to form. These planets are able to carve out a gap in the dust and gas components of a protoplanetary disc (Paardekooper \& Mellema 2006), developing the pressure maximum, and large dust grains, which would otherwise radially migrate inwards, are trapped. 
Substructures have been observed in the protoplanetary discs surrounding stars with a wide range of different stellar parameters. Previous works have mostly focused on individual objects, or surveys have been conducted on specific regions (targeting both uniform and structured discs). More recently, van der Marel et al. (2019) focused solely on stars surrounded by protoplanetary discs featuring rings, while the Disk Substructures at High Angular Resolution Project (DSHARP) looked at 20 nearby discs to characterize the range of substructures seen in the dust spatial distribution (Andrews et al. 2018b). Long et al. (2018) have also recently focused on discs in Taurus featuring substructure.

In this work, we study the different substructures found in 56 protoplanetary discs observed with ALMA. There are a range of substructures seen in the discs and the stars studied cover a wide parameter range of both intermediate- and low-mass stars. Our 56 discs were chosen from the complete sample of discs observed by ALMA thus far. This is discussed further in Section 2, where we also present our sample. We discuss any biases in our sample in Section 3. This is followed by Section 6.1, where the properties of our sample are both derived here and obtained from literature. We present a new morphology scheme to classify the substructure seen in protoplanetary discs in Section 5. The main results of this paper are then presented in Section 6 and discussed in Section 7. We finally conclude in Section 8.

\section{DATA}

The ALMA archive has been searched to find protoplanetary discs that feature different morphological structures seen in the millimetre dust continuum. The continuum product images of 793 targets from cycles 0 to 5 were looked at. The full sample of images we have looked at is shown in Table A1 in Appendix A. The project code for each observation can also be found in the table. The images obtained from the archive are of 793 unique systems, and feature both young pre-stellar discs and older debris discs.

Protoplanetary discs that are spatially resolved and show clear substructure in millimetre dust were chosen. We confirm that the discs are resolved by comparing the beam size of the observations to the disc size. In order to be classified as having substructure, the discs had to feature either a cavity or gap of some sort or a pile-up of dust grains. We are interested in asymmetric substructure that may be seen in the dust continuum of the mm-dust disc. We have chosen to exclude debris discs from our sample, including the well-studied discs surrounding HR4796A, HD107146, and HD181327. This was done as they are much older than the discs in our sample and the ALMA Archive is much less complete for these older discs (see Section 6.2). Similarly, we have excluded young, embedded discs such as Elias 2-24 and HL Tau as they may still be surrounded by an envelope. If no previous observations have found an object to be embedded, we discard it if its age is determined to be less than 0.5 Myr. These young objects are discarded as the ALMA Archive is much less complete for these discs. We have only obtained the final, calibrated continuum products of each observation and any images that have undergone further processing will be discussed in Section 5.1 below.

An absence of substructure may be seen in many of the discs due to a number of reasons. First, many objects observed by ALMA, unless explicitly targetted, have only been observed once. The detection of substructure can depend upon the observational waveband, resolution, and sensitivity. Therefore, many discs may feature substructure, but this has not been detected due to the incorrect conditions under which the observations were conducted. This is discussed further in Section 3.

Thus, we have a large sample of protoplanetary discs observed by ALMA, during cycles 0 to 5 , that currently shows resolvable substructure. We acknowledge that our sample is not complete with regards to all protoplanetary discs featuring substructure, as some discs in the archive may have substructure that remains unresolved. It is, however, a large enough sample from which conclusions can be drawn.

The sample that we are left with consists of 56 protoplanetary discs across the first six cycles of ALMA observations. The observations for each disc were conducted at either Band 6 or Band 7 (1.3 or 0.8 $\mathrm{mm}$, respectively) with three exceptions. Observations of 2MASS J16152023-3255051 (hereafter J16152023) and HD36112 were conducted at Band $9(0.4 \mathrm{~mm})$, while observations of HD163296 were conducted at Band $8(0.6 \mathrm{~mm})$. The complete sample of discs is shown in Table 1.

The continuum product files available on the ALMA Archive are often of a lower quality than the final published data. We compare the product files to the published data on each source and find that the quality of the data, with regards to the final rms and recovered total flux, improves by less than an order of magnitude. No additional substructure is revealed between the archive product files and the published data. Therefore, additional processing has not been done in order to improve the quality of the product files as this is not needed for the analysis conducted in this work.

The archival ALMA observations were taken at a range of resolutions; all sub-arcsecond. We further discuss the detectability of substructure in Section 3.1. The smallest angular scale we can resolve in our sample is in the DM Tau disc, with a resolution of 0.02 arcsec ( 2.9 au at $145 \mathrm{pc}$ ), while we can only resolve down to 0.77 arcsec (123.6 pc at $160 \mathrm{pc}$ ) in the disc of 2MASS J16230923-2417047. Also, the discs are located at a range of distances and the observations were conducted with varying sensitivities. As a result, any statement on the detectability of substructure based on flux would be meaningless.

\section{OBSERVATIONAL BIASES OF ALMA}

The analysis conducted in this work is subjected to several observational and selection biases. We discuss this further in the sections below.

\subsection{Angular resolution}

The angular resolutions of the discs studied in this work are shown in Table 2. These discs were observed with a higher resolution than the majority of the ALMA discs (see Table A1 for the angular resolution of all the ALMA protoplanetary discs observed during cycles 0-5). We have plotted a histogram of the angular resolutions with which the individual protoplanetary discs in our sample were observed (see Table A1). This is shown in Fig. 1.

We have looked at 793 protoplanetary discs, of which we have identified 56 that show substructure. As discussed above, we have omitted known young, embedded discs as well as debris discs. The majority of the discs we have classified here (46/56 $\simeq 80$ per cent) were observed with a resolution higher than 0.22 arcsec. This limit is indicated by the red dashed line on Fig. 1. From the complete sample of ALMA discs looked at, 171 discs were observed with a resolution of $0.22 \operatorname{arcsec}$ or better. Therefore, $\sim 27$ percent of the discs observed with ALMA with a resolution 0.22 arcsec or greater have resolvable substructure. 
Table 1. Literature properties of the sample studied in this work. The references correspond to the luminosity and temperature, respectively. All distances have been obtained from Gaia DR2 (Gaia Collaboration 2018) with the exceptions of 2MASS J05052286+2531312 and RY Tau which were obtained from Kenyon, Gómez \& Whitney (2008) and (Garufi et al. 2019), respectively (see the text for details). Stellar masses are calculated in Section 4.1.

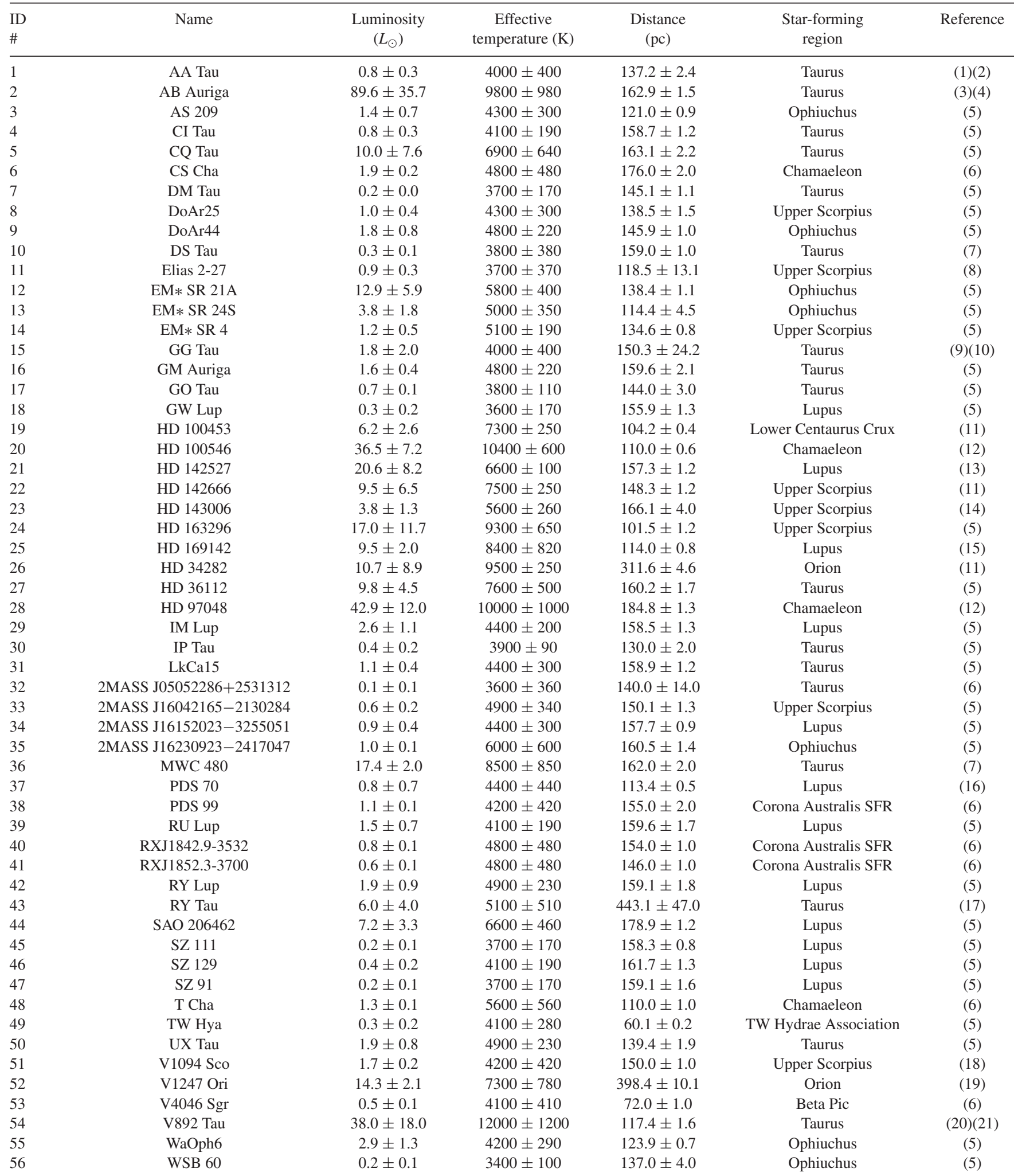

Note. The distance measurement for RY Tau has been obtained from Garufi et al. (2019). See the text for further details. References: (1) Grosso et al. (2007), (2) Schneider et al. (2015), (3) Monnier et al. (2006), (4) Isella, Testi \& Natta (2006), (5) Andrews et al. (2018a), (6) Francis \& van der Marel (2020), (7)Long et al. (2018), (8) Isella, Carpenter \& Sargent (2009), (9) White et al. (1999), (10) Dutrey et al. (2016), (11) Fairlamb et al. (2015), (12) van den Ancker et al. (1997), (13) Mendigutía et al. (2014), (14) Andrews et al. (2018b), (15) Fedele et al. (2017), (16) Metchev, Hillenbrand \& Meyer (2004), (17) Bertout, Siess \& Cabrit (2007), (18) van Terwisga et al. (2018), (19) Kraus et al. (2013), (20) Berrilli et al. (1992), (21) Manoj et al. (2006). 
Table 2. The values derived in this work. Columns 3 and 4 show the stellar ages and masses derived using the stellar tracks from Siess et al. (2000) and Baraffe et al. (2015). Column 5 shows the classification assigned to the protoplanetary disc. The radius of the disc that contains 68 per cent of the total disc flux is shown in Column 6. Columns 7 and 8 show the spatial resolution and continuum sensitivity for each ALMA observation, while column 9 shows the ALMA project code.

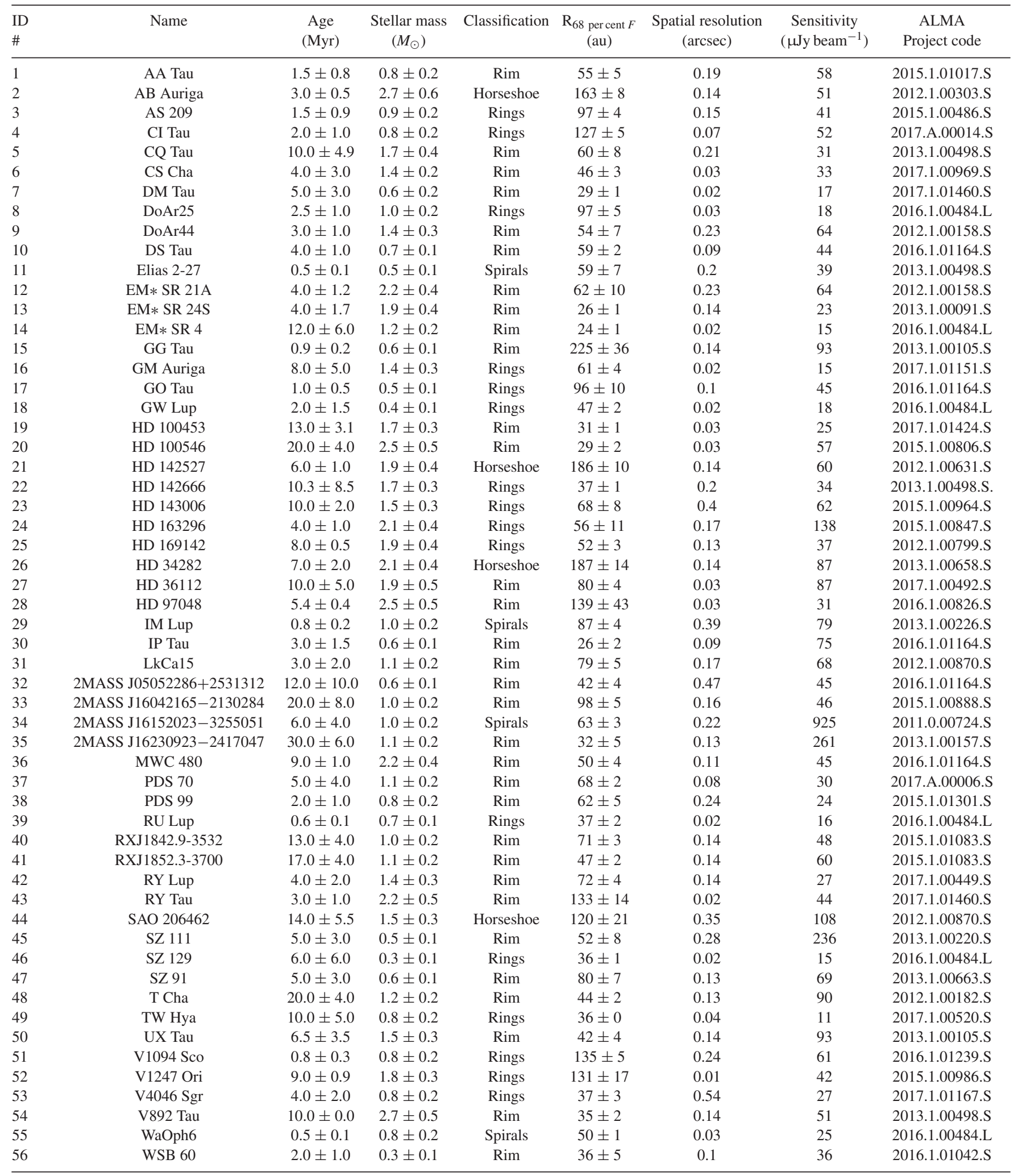




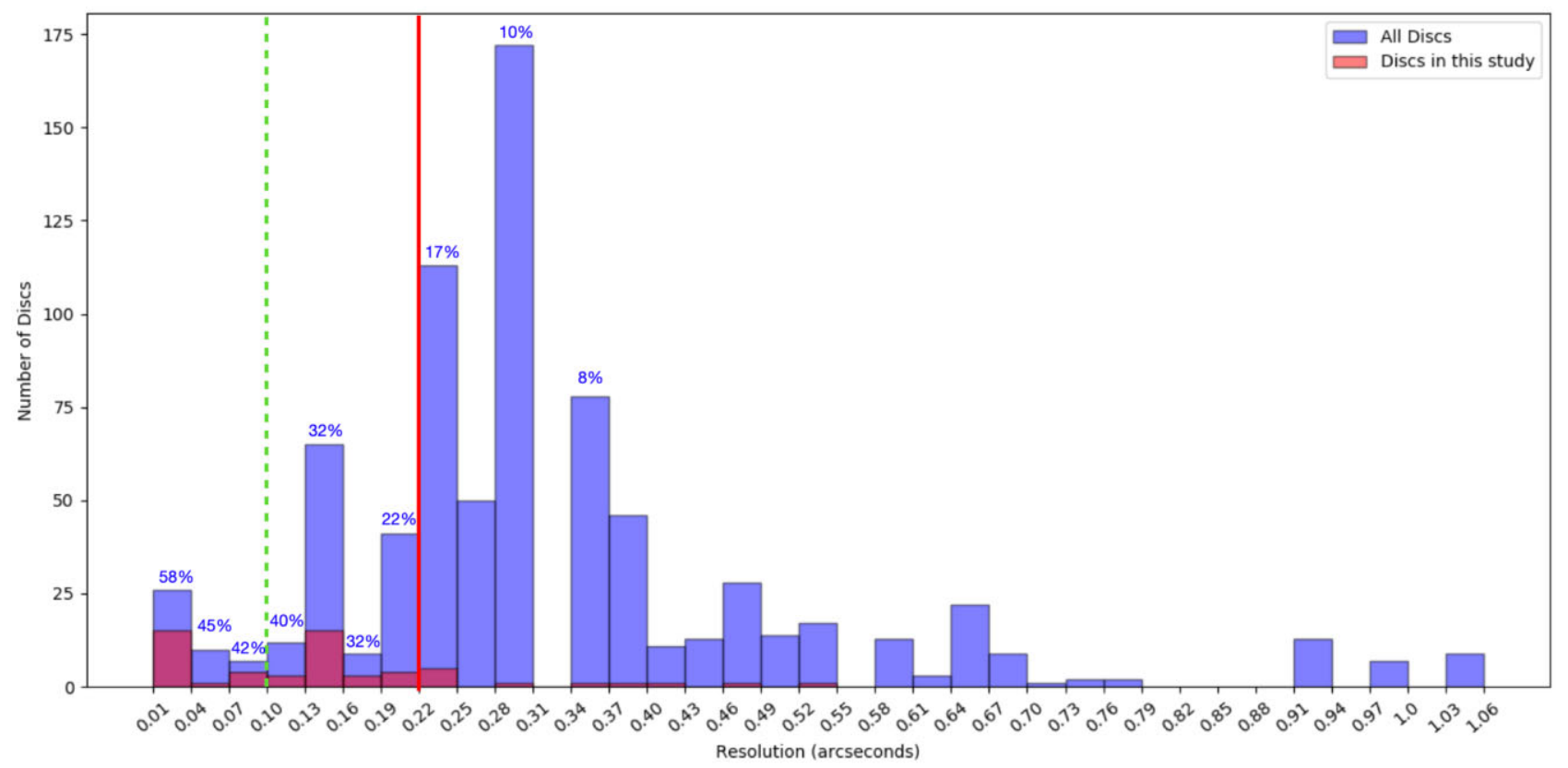

Figure 1. The resolutions of the entire sample of protoplanetary discs we have looked at in the ALMA Archive. Details of the discs can be found in Table A1. The discs have been binned with resolutions of 0.03 arcsec. The red, solid line indicates the resolution limit below which the majority of the discs featuring substructure were observed at $(46 / 56 \simeq 80$ per cent). The green, dashed line indicates a resolution limit of 0.1 arcsec, below which is considered 'high resolution' with ALMA. We have highlighted, in pink, the discs identified as containing substructure and studied in this work. The percentages above each bin indicate the fraction of discs showing substructure at each resolution and greater.

Cieza et al. (2019) conduct a survey of the discs within Ophiuchus using ALMA at a resolution of 0.2 arcsec. Observations were able to resolve 60 protoplanetary discs, of which 12 showed substructure (eight clear, and four probable detections). This is 20 per cent of their resolved discs, which agrees with the finding here that $\sim 27$ per cent of discs show substructure when observed at a resolution limit of 0.22 arcsec.

A resolution of 0.22 arcsec is still considered relatively moderate with ALMA. Studies explicitly targeting transition discs and discs with known substructure aim for resolutions of $\approx 0.1$ arcsec or better. A total of 52 protoplanetary discs observed with ALMA at a resolution of 0.1 arcsec or better have been looked at in this work. This limit is shown as the green line on the histogram (Fig. 1). Of these 52 discs, 22 have been shown to have substructure and have been classified here. This is approximately 42 per cent of the discs.

Long et al. (2019) studied 32 protoplanetary discs in Taurus with ALMA using a resolution of $\approx 0.12$ arcsec. They found that just under half of the discs showed dust gaps and rings. In agreement with the 42 percent we have found here for the same resolution limit. The DSHARP project, however, has found that all the discs they observe at a very high resolution of $\approx 0.035$ arcsec feature substructure. It should be noted, however, that the DSHARP project was a targeted survey and only looked at discs that had previously showed substructure. For a resolution limit of $\$ 0.04$ arcsec, we find that the fraction of discs showing substructure increases to 60 per cent.

\subsection{Sensitivity}

The continuum sensitivities of the discs studied in this work are shown in Table 2, with Table A1 showing the sensitivity of all the ALMA protoplanetary discs observed during cycles 0-5. A histogram of the sensitivities used to observe the discs in this work has been plotted in Fig. 2.

The majority of our discs $(53 / 56 \simeq 85$ per cent $)$ that show substructure were observed with a sensitivity greater than $140 \mu \mathrm{Jy}_{\text {beam }}{ }^{-1}$. A second histogram showing the discs with sensitivities greater than this can be seen in Fig. 2. From the complete sample of ALMA discs looked at, 308 discs were observed with a sensitivity of $140 \mu \mathrm{Jy}_{\text {beam }}{ }^{-1}$ or greater. Therefore, substructure can be seen in $\sim 17$ percent of the discs observed with ALMA when observed with a sensitivity of $140 \mu \mathrm{Jy}_{\text {beam }}{ }^{-1}$ or greater. This value agrees with the work of Cieza et al. (2019), who find that 20 percent of their discs show substructure when observed with a sensitivity of $150 \mu \mathrm{Jy}_{\text {beam }}{ }^{-1}$.

The 32 protoplanetary discs observed by Long et al. (2019) had an average sensitivity of $50 \mu \mathrm{Jy}_{\text {beam }}^{-1}$. We have indicated this sensitivity limit on Fig. 2 with a pink dashed line. The number of discs showing substructure below this limit is 31 ; this is $\sim 25$ per cent out of a total of 126 discs observed by ALMA. Long et al. (2019) were able to detect substructure in just under half of their discs.

Similar to the effect of increasing the resolution, increasing the sensitivity limit to $20 \mu \mathrm{Jy}$ beam $^{-1}$ results in 8 out of 23 discs featuring substructure ( $\sim 35$ per cent). This limit is indicated by the blue, solid line on Fig. 2. At an average sensitivity limit of $17 \mu \mathrm{Jy}_{\text {beam }}^{-1}$, the DSHARP project was able to detect substructure in all the discs they observed (Andrews et al. 2018b).

\subsection{Selection biases}

We have studied 56 protoplanetary discs that cover a wide parameter space. Both low- and intermediate-mass stars have been looked at, with a wide range of ages, temperatures, and luminosities. A plot of the relation between the stellar masses of our sample and their 


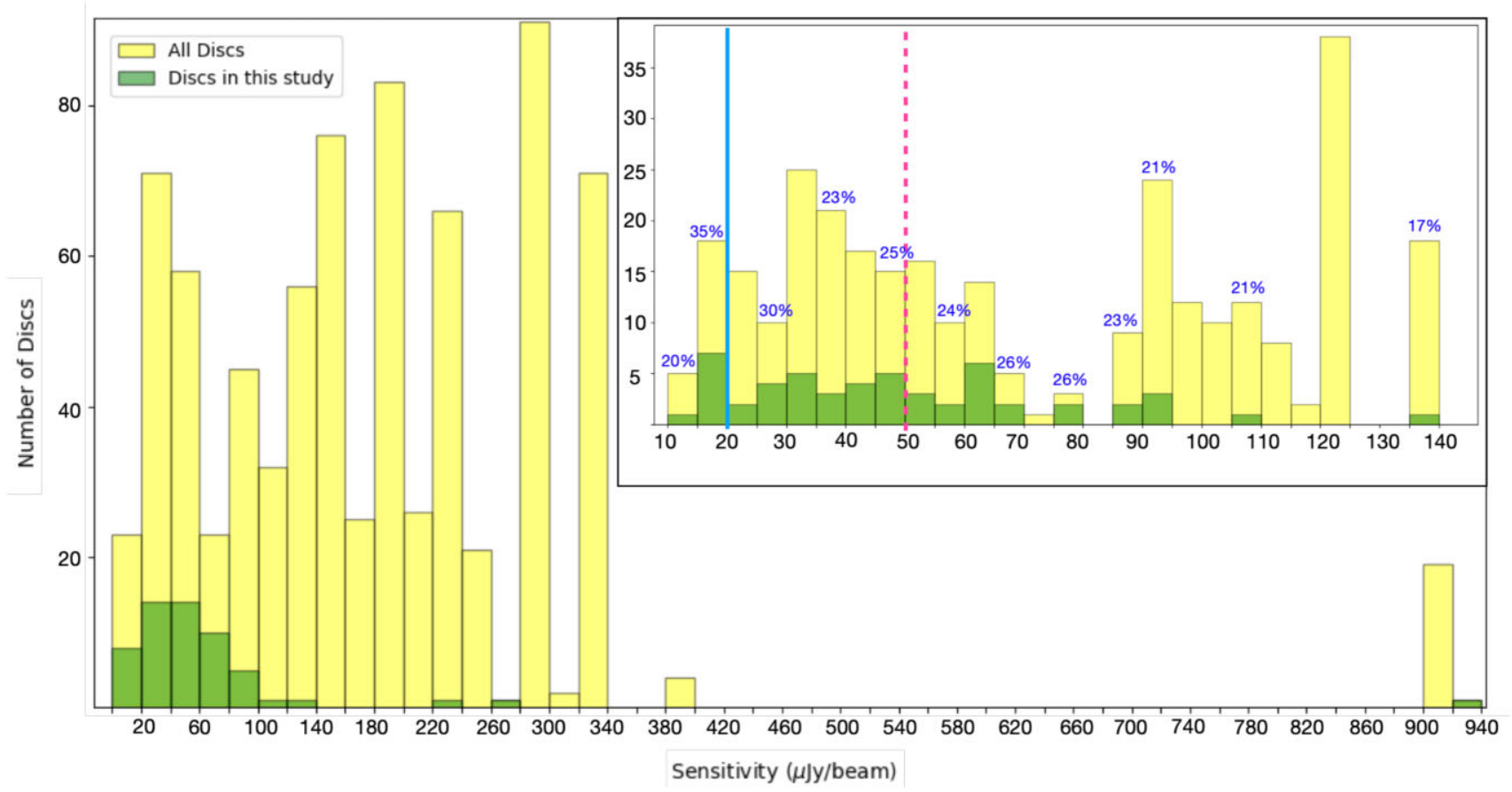

Figure 2. The continuum sensitivities of the entire sample of protoplanetary discs we have looked at in the ALMA Archive. Details of the discs can be found in Table A1. The discs have been binned with sensitivities of $20 \mu \mathrm{Jy}_{\text {beam }}{ }^{-1}$. The graph insert shows a zoomed-in version of the original figure binned to $10 \mu \mathrm{Jy}_{\text {beam }}{ }^{-1}$. The pink dashed, and blue solid lines indicate the mean sensitivities of the discs observed by Long et al. (2019) and Andrews et al. (2018b), respectively. The percentages above each bin indicate the fraction of discs showing substructure at each sensitivity and greater.

corresponding age has been made in order to determine if there are any observational biases in our sample (see Fig. 3).

There is a lack of old low-mass stars that show protoplanetary substructure as well as significantly fewer old high-mass stars. Although old low-mass stars have been previously observed (Barenfeld et al. 2016), deep, high-resolution observations have not been conducted with ALMA. This may indicate why there is a lack of old low-mass stars in our sample.

We also see that there are significantly fewer young, high-mass stars in our sample. This could be attributed to the fact that highmass stars evolve on a much quicker time-scale than low-mass stars. They can evolve to the post pre-main-sequence (PMS) phase while still being embedded and actively accreting (Beuther et al. 2007). Therefore, imaging a protoplanetary disc around a high-mass star would be problematic.

Our sample contains more low-mass stars than intermediate-mass stars. However, it is known that low-mass stars are more common than intermediate- and high-mass stars, as would be expected from a standard Salpeter-like initial mass function (Salpeter 1955; Kroupa 2001; Chabrier 2003). Therefore, any results showing the commonalty of substructures around low-mass stars compared to high-mass stars may be a selection bias.

\subsection{Comparison to full ALMA sample}

A total of 793 protoplanetary discs were looked at in this work. Omitting young, embedded discs, as well as debris discs, we have identified 56 discs that show some kind of substructure, which is around 7 per cent of all discs. This is in contrast to Long et al. (2019) and work by the DSHARP project (Andrews et al. 2018b), who find that at least 50 percent of the discs studied show substructure. As discussed in Section 3.1 above, this is due to the majority of ALMA observations being conducted at lower resolutions than studied in these works.

The 793 protoplanetary discs studied here mainly surround Class I, II, and III young stellar objects. However, the protoplanetary disc surveys conducted by ALMA thus far have focused mainly on Class II discs from several star-forming regions. These include Chamaeleon I (Pascucci et al. 2016), Lupus (Ansdell et al. 2016, 2018), Ophiuchus (Cox et al. 2017; Cieza et al. 2019), the Orion Nebula Cluster (Eisner et al. 2018), $\sigma$ Ori (Ansdell et al. 2017), Taurus (Akeson \& Jensen 2014; Akeson et al. 2019; Long et al. 2018, 2019), and Upper Scorpius (Barenfeld et al. 2016).

Although these surveys focused on Class II protoplanetary discs, the star-forming regions have a range of ages. Therefore, the Class II discs in the ALMA archive are at different evolutionary stages. The sample of 56 discs studied here is representative of that. We have young stellar objects from 11 different star-forming regions, with a range of ages (see Table A1 in Appendix A). Also, there is a diverse range of calculated ages for the young stellar objects, the youngest being Wa Oph $6(0.5 \pm 0.1 \mathrm{Myr})$ and the oldest being J160230923 (30.0 $\pm 5.0 \mathrm{Myr}$ ). Therefore, we have studied systems with a comparable range of Class II evolutionary states as that found in the ALMA Archive.

\section{PROPERTIES OF SAMPLE}

A literature search has been conducted to obtain the temperature and luminosity of each host star. The luminosities have been scaled to the Gaia DR2 distances (Gaia Collaboration 2018). Where the error is not given in the literature for the temperature, we make an estimate of the uncertainty. The typical error on the quoted values is 6 per cent, therefore we use this and make a conservative estimated error of 10 per cent for temperatures without an uncertainty value. No Gaia 


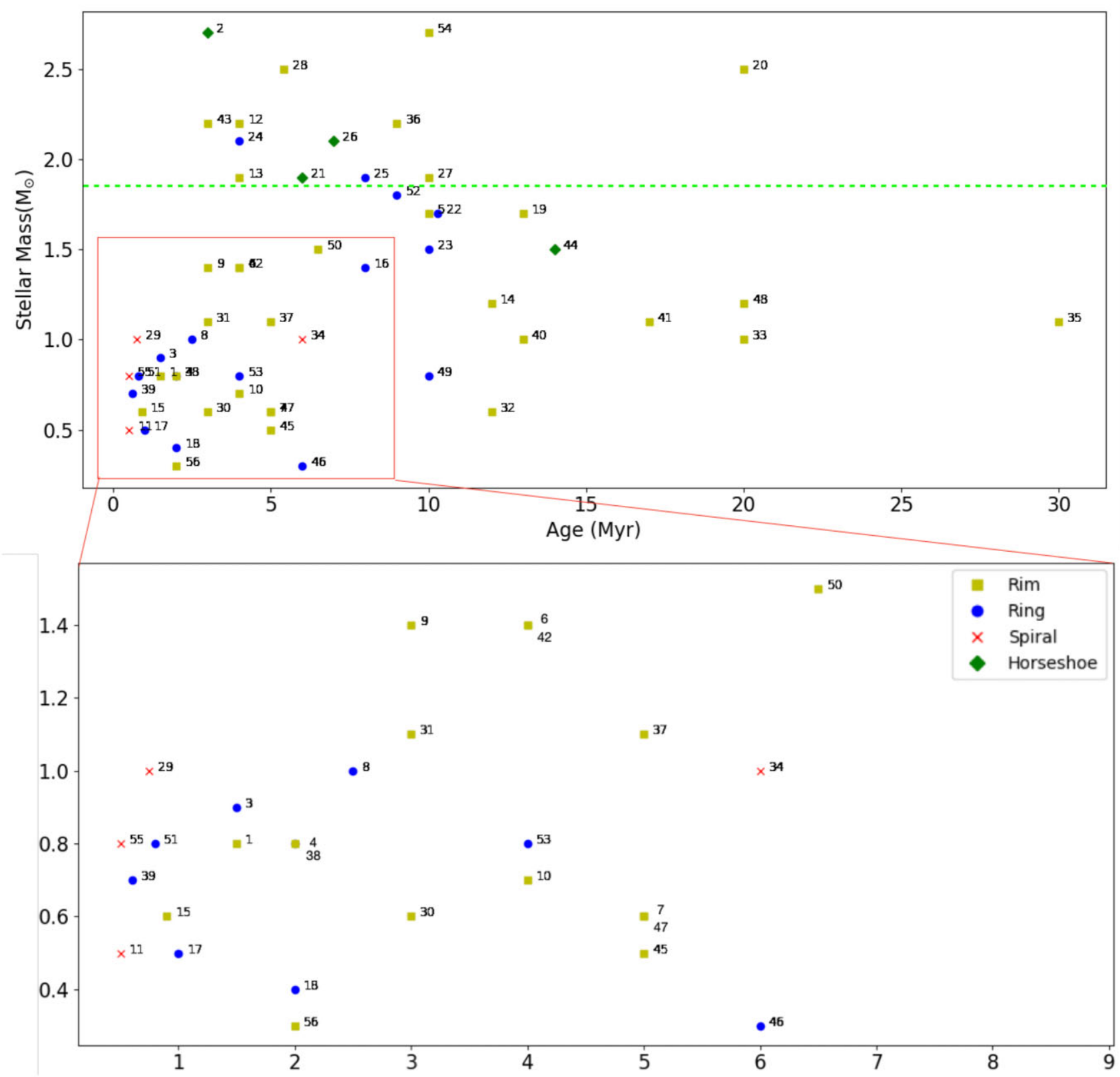

Figure 3. Stellar mass with age. The coloured markers indicate the category assigned to each protoplanetary disc in Section 5 . The green dashed line indicates the limit between low-mass and intermediate-mass, as defined in Section 6.1.

DR2 distances were available for 2MASS J05052286+2531312. We make a distance estimate of $140.0 \pm 14.0 \mathrm{pc}$ for this object based on its location in the Taurus molecular cloud. We have assumed a conservative error estimate of 10 percent for this distance.

An HR diagram of the sample has been plotted in Fig. 4. It is evident in the diagram that our sample uniformly covers both stellar luminosity and temperature. The zero-age main sequence (ZAMS) has also been plotted on the HR Diagram (ZAMS from Siess, Dufour \& Forestini 2000). The discs have been plotting with different symbols according to the classification they have been given in this work. This will be discussed further in Section 5.

The 56 host stars have luminosities spanning from 0.1 to $90 L_{\odot}$ and temperatures from 3400 to $12000 \mathrm{~K}$, covering a wide parameter range of low- and intermediate-mass stars. We refer to host stars with mass $\mathrm{M}<1.8 M_{\odot}$ as low-mass objects. Intermediate objects are defined as those with mass $1.8<M_{\odot}<8$, while objects with mass greater than $8 M_{\odot}$ are referred to as high-mass objects. The lower mass limit of $1.8 M_{\odot}$ for the intermediate stars comes from Simon et al. (2002). The lower mass limit for high-mass stars $\left(8 \mathrm{M}_{\odot}\right)$ originates from the minimum mass required to produce a Type II supernova (Zinnecker \& Yorke 2007). The stars in our sample are located in a number of different star-forming regions (SFR), at a range of distances.

The Gaia DR2 distances agree to within 20 per cent of the previous literature values for the majority of sources. The DR2 distance to HD169142 was determined to be $114.0 \mathrm{pc}$, compared to its previous distance of 145.0 pc (Manoj et al. 2006). Other examples include 

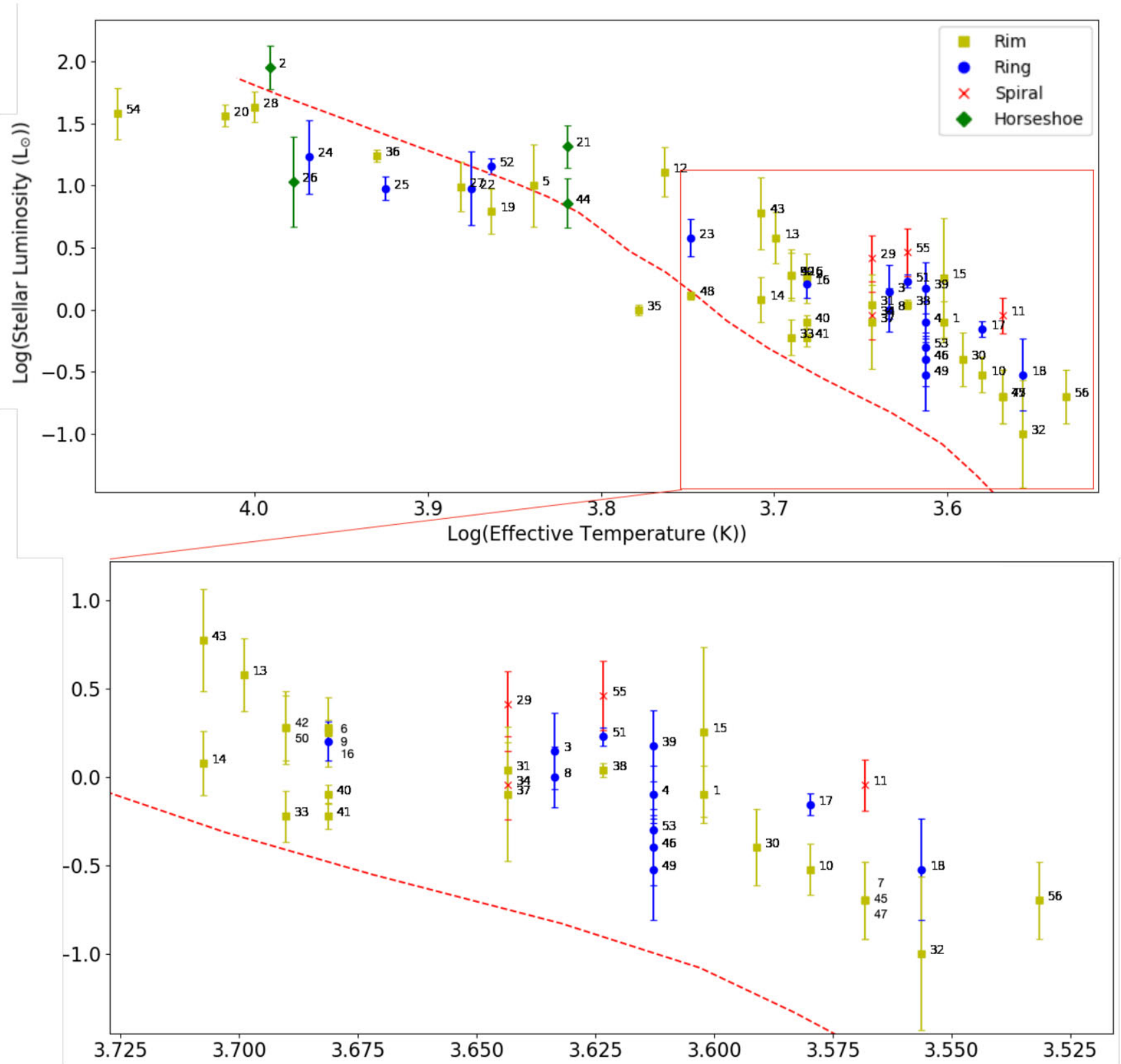

Figure 4. An HR diagram of the sources in our sample. The red dashed line marks the ZAMS using the model of Siess et al. (2000). The discs have been labelled and their identities can be found in Table 1. The coloured markers indicate the category assigned to each protoplanetary disc (see Section 5).

SZ111, which changed from 200.0 to $158.3 \mathrm{pc}$, and CQ Tau, 100 to 163 pc. The estimated distance to the Class II T-Tauri star RY Tau has changed drastically, from 140 pc (Agra-Amboage et al. 2009) to 177 pc in Gaia DR1 to $443.1 \pm 47.0$ pc in Gaia DR2 (Gaia Collaboration 2018). This discrepancy was discussed by Garufi et al. (2019). Therefore, we adopt their value of $133_{-30}^{+55} \mathrm{pc}$ obtained from HIPPARCOS data (ESA 1997) The majority of the discs observed with ALMA are located in well-surveyed star-forming regions and have distances less than $\sim 200 \mathrm{pc}$.

All but eight systems in our sample are single-star systems. UX Tau A and HD100453 both have companions orbiting at a radius $>100$ au and are surrounded by a circumprimary disc (Tanii et al. 2012; Wagner et al. 2018). Circumbinary discs surround HD142527, CS Cha, DS Tau, V4046 Sgr, V892 Tau, and GG Tau (with GG Tau A being a triple-star system, Di Folco et al. 2014) (Smith et al. 2005;
Fukagawa et al. 2006; Guenther et al. 2007; Rodriguez et al. 2010; Akeson \& Jensen 2014). With such a limited sample of multiple star systems, we have not investigated trends relating to disc morphology and multiplicity.

\subsection{Determining stellar ages and masses}

Individual stellar ages and masses were determined for the host stars using the new luminosities and the models of Siess et al. (2000) and Baraffe et al. (2015). The models of Baraffe et al. (2015) are only applicable for stars $<1.4 \mathrm{M}_{\odot}$, therefore we use the models of Siess et al. (2000) for any star with a mass larger than this. Evolutionary tracks were plotted on to the HR diagram (Fig. 4) and the stellar mass and ages were interpolated. Errors on the ages and masses are derived using the associated luminosity error for each star. For 
reference, we show the HR diagrams with plotted evolutionary tracks in Appendix B.

The derived ages are given in Table 2. It should be noted that this method of determining the ages and masses of PMS stars is quite uncertain. This is due to the luminosities of some stars having large errors, resulting in a large uncertainty range for the ages and masses. Therefore, the derived values from this method are only an estimate and should be treated with caution. The estimates of the stellar ages are later used to order the protoplanetary discs according to age (see Section 6).

We compare the ages derived here to the estimated age of the star-forming region each star belongs to. We find that the ages we have derived generally agree with the estimated ages of the starforming region within $3 \sigma$. The ages of the star-forming regions can be found in Table B1. We find that the derived ages of the objects that do not agree with the age of the star-forming region ar either very young or very old objects. Therefore, the stellar isochrones may be underestimating or overestimating the ages for these stars.

\subsection{Determining disc radii}

For a quantitative analysis of the structure of the protoplanetary discs, we calculate the dust disc radii. As the observations were taken with different resolutions and sensitivities, we calculate the radius containing 68 per cent of the total flux for each disc (see Tripathi et al. 2017 for a more detailed explanation of this method). The radius of each disc can be found in Table 2. This method for determining the effective radius of the disc has previously been utilized in a number of works. We compare our derived radii to literature values of the radii containing 68 percent of the total flux for the discs where this has been calculated. We find that the radii calculated here agree with the literature values within $3 \sigma$ (Tripathi et al. 2017; Ansdell et al. 2018; Long et al. 2019). We make use of the derived radii in Sections 6.2 and 6.3.

\section{CLASSIFYING PROTOPLANETARY DISCS}

Garufi et al. (2018) studied the appearance of protoplanetary discs (in morphology and spatial extent) in scattered light. Stellar and disc properties were calculated and related to seven categories defined in the study: Ring, Spiral, Giant, Rim, Inclined, Faint, and Small discs. Following a similar approach, we classify our discs into four categories based upon their appearance and morphology. The characteristics of the four categories are outlined below and summarized in Fig. 5.

Rings: Protoplanetary discs that feature bright, concentric rings have been classified as ringed discs. Between the rings are gaps devoid of gas and/or dust. A minimum of two concentric rings need to be seen in order for a disc to be classified as ringed. Examples of these discs that have been observed with ALMA include GM Auriga (Macías et al. 2018) and HD169142 (Fedele et al. 2017). These discs may also feature a central cavity, as can be seen in GM Auriga.

Rims: The majority of the discs that have been studied in this work feature a single bright rim at the edge of a large disc cavity. These rims have been seen in both intermediate-mass and low-mass young stellar objects including DM Tau, LkCa15, and 2MASS J160421652130284 - hereafter J16042165 (Pinilla et al. 2018). Disc rims can also be seen in protoplanetary discs that have been classified as ring or horseshoe. However since the rim is not the most prominent feature, these discs have been sorted in other categories. Furthermore, rims can be seen in some discs as such at the present angular resolution. As we will discuss later in the paper, further observation of rim discs

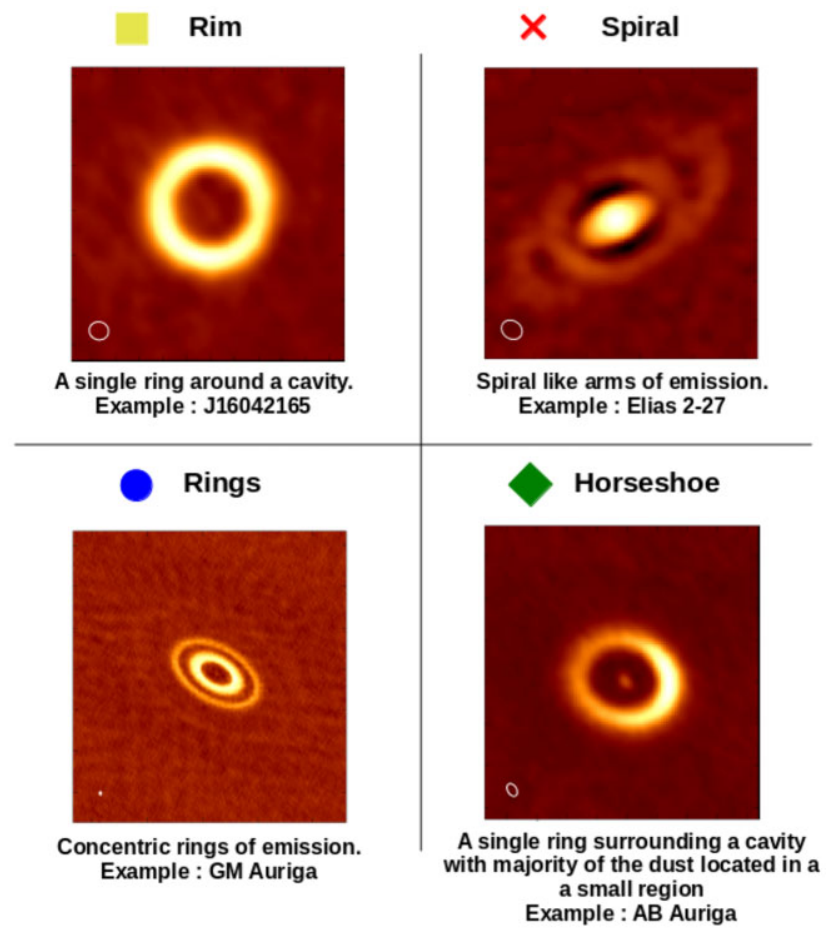

Figure 5. Examples of protoplanetary discs from each category defined in this work. More detail about each category is given in the text.

may place them in alternate categories. Since we are only focusing on the continuum emission from the protoplanetary disc, the cavity needs to be void of only dust in order to be classified as a rim.

Horseshoe: Horseshoe-shaped discs are similar to rim discs; a single ring of dust surrounding a large cavity. However, the majority of the dust in horseshoe discs is located in a small region of one side of the disc. An example of a system containing a horseshoe-shaped protoplanetary disc is HD142527 (Casassus et al. 2015), where the dust in the protoplanetary disc resembles a crescent or horseshoe shape.

Spiral: Discs which feature spiral-like arms of continuum emission are classified as spiral discs. This structure has rarely been seen in protoplanetary discs as of yet, the first being Elias 2-27 (Pérez et al. 2016).

All of our chosen discs are resolved and show substructure, therefore we have omitted the Faint category of discs used by Garufi et al. (2018). We have also not used the Small disc category as all of our discs have a radius larger than 20 au. Garufi et al. (2018) define discs that show faint arm-like structures on large scales as Giant. These structures are only seen in discs with a radial extent $\gg 100$ $\mathrm{au}$. The structures seen in these discs are faint and could even be attributed to broken rings rather than spiral arms. Therefore, we have removed the Giant category and introduced the Horseshoe category which has been outlined above.

The discs have been classified based on the most prominent morphological feature seen in the sub-mm dust emission. Some discs like CQ Tau could be defined as either a horseshoe disc or a rim disc. We have chosen to classify this disc as a rim disc since this is the most prominent feature. RY Lup also appears to have a horseshoe-like morphology. However this disc is nearly edge on with an inclination angle of $\approx 70 \mathrm{deg}$ (Langlois et al. 2018). Therefore, the horseshoe-like morphology may just be an observational effect. Therefore, we have placed this disc in the Rim category. We strongly 


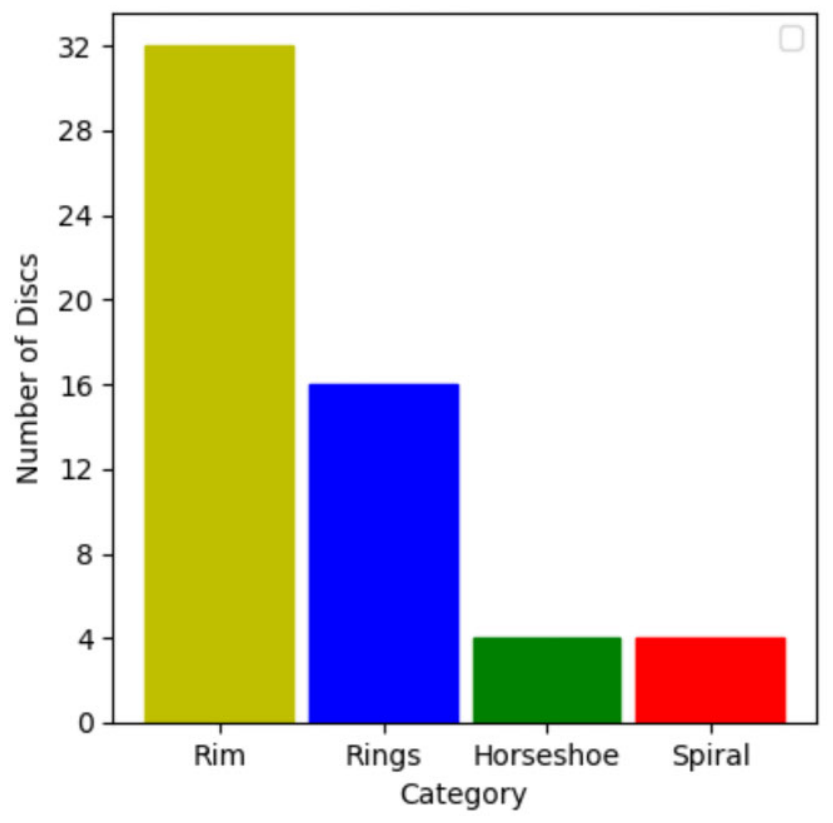

Figure 6. 56 protoplanetary discs have been studied in our sample. Rim and Ringed protoplanetary discs are the most common discs that have been observed with ALMA. Horseshoe and Spiral discs are the least common types.

acknowledge that some discs can fit into multiple classifications. The classification for each source can be found in Table 2 .

\subsection{Unsharp Masking Filtering}

ALMA observations of Elias 2-27 by Pérez et al. (2016) revealed spiral density waves in the protoplanetary disc. The technique of Unsharp Masking Filtering was performed on the images in order to remove the large-scale disc emission and highlight the spiral structure of the disc. The observations of Elias 2-27 from Pérez et al. (2016) were obtained from the ALMA Archive and the original observation was smoothed with a 2D Gaussian of 0.33 arcsec full width at halfmaximum (FWHM). This image was then scaled by a factor of 0.87 before subtracting it from the original image.

We applied a similar process to the ALMA observations of J16152023 and Wa Oph 6 as faint spiral substructure could be seen. The Filtering was applied to J16152023 and Wa Oph 6 in order to highlight the spiral structures that can be seen in the disc. These discs were smoothed with a Gaussian of FWHM 0.33 arcsec; J16152023 was scaled by a factor of 0.87 , while Wa Oph 6 was scaled by 0.67 . The Filtering was performed in order to bring out the fainter structures of the disc; this then allowed us to better classify these protoplanetary discs. No other science was performed using these filtered images

\section{RESULTS}

\subsection{Prevalence of each substructure}

The number of discs in each category can be seen in Fig. 6. Of the 56 discs studied in this sample, Rim discs appear to be the most populous category in our sample featuring 32 discs. 16 discs have been classified as having rings. Horseshoe and Spiral discs are least populous, with four discs in each category. Although the majority of our sample have been classified as a Rim disc, it should be noted that cavities are the easiest substructure to be detected with ALMA. And as a result, the large number of Rim discs may be a selection effect due to extensive interest in them. This is further discussed in Section 7.1.1 below. While horseshoe-shaped discs have been placed in their own category, they all feature a single ring of emission on the edge of a large cavity and could also be classified as Rim discs. This would increase the number of Rim discs to 36 , more than 60 per cent of our sample, intensifying the notion that the presence of a Rim seems to be a common feature in the current population of protoplanetary discs observed at mm-wavelengths.

We now investigate the properties of the host stars of each disc category. From Fig. 4 we can see that Horseshoe-shaped discs are most commonly found around intermediate-mass stars. These stars are all Herbig Ae/Be type stars, with their high effective temperatures ( $T_{\text {eff }}>6000 \mathrm{~K}$ ). The lack of Horseshoe-shaped protoplanetary discs around low-mass stars may indicate that the formation mechanism responsible for forming a pile-up of dust grains on one side of the disc preferentially occurs in higher mass stars (van der Marel et al. 2021).

Spiral discs surround just four host stars in our sample. All systems in this category are single-star systems and are Class II T-Tauri-type stars with stellar masses $\leq 1.0 M_{\odot}$. Due to the small number of both Horseshoe and Spiral discs, we cannot make any real conclusions about the characteristics of the host stars that these discs surround.

\subsection{Structure versus age}

To study whether the morphological structures seen in protoplanetary discs evolve with age, we have ordered the discs studied in this work according to age. We have used the ages derived in Section 4.1 using the evolutionary tracks from Siess et al. (2000) and Baraffe et al. (2015). Figs C1, C2, C3, and C4 in Appendix C show the Rim, Ring, Horseshoe, and Spiral discs ordered according to age. Images have been scaled to $500 \times 500 \mathrm{au}$. Stars with a $\dagger$ symbol in the lower left corner have been scaled to $1000 \times 1000 \mathrm{au}$. This has been done to enable us to compare the spatial extent of the discs.

\subsubsection{Rims and ring}

The Rim discs, ordered by age, can be seen in Fig. C1. The ages of the stars surrounded by a Rim protoplanetary disc vary quite widely. The youngest disc is a T-Tauri star with an age of $0.9 \pm 0.2 \mathrm{Myr}$, while J16230923 is the oldest disc with an age of $30.0 \pm 6.0 \mathrm{Myr}$. Furthermore, from Fig. 3, we can see that all of the discs with an age greater than $10 \mathrm{Myr}$ (except one Horseshoe disc) are surrounded by a Rim. Therefore, a Rim appears to be a long-lasting structure of a protoplanetary disc, whereas a Spiral or Ring is a shorter lived structure. The intermediate-mass stars with Rims appear in the later stages of the evolutionary plot. However, this may simply be an observational bias as there are very little observations of old lowmass stars.

Fig. C2 shows the Ring discs ordered by age. There does not appear to be a correlation between the age of the system and the radial extent of the disc. RU Lup is the youngest Ring disc we have classified, while HD 142666 is the oldest. Each low-mass star is scaled to $500 \times 500 \mathrm{au}$, therefore we can see that the radial extent of the disc does not depend on the age of the system.

The spatial extent of the Rim and Ring discs does not appear to be correlated with age. We test this quantitatively by plotting the disc radius against the derived stellar age (values in Table 2). This plot is shown in Fig. 7. The discs plotted do not feature a spiral or horseshoe 


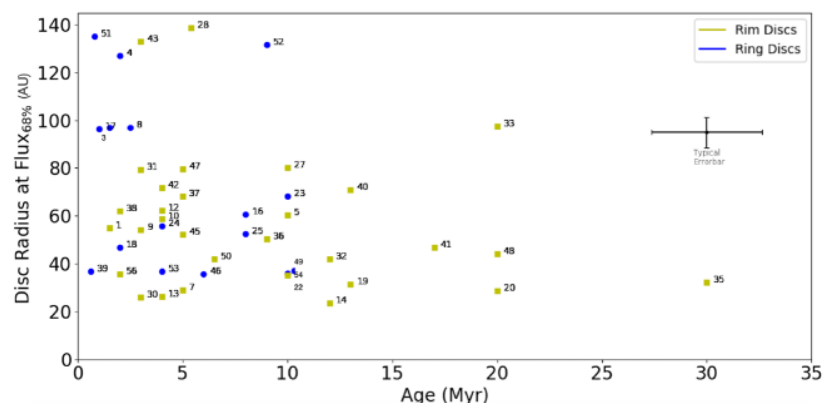

Figure 7. The radii of the protoplanetary discs containing 68 per cent of the total flux against the stellar age. The yellow markers indicate the Rim discs and the blue markers indicate the Ring discs.

morphology as we have a low number of these discs. We have also omitted the circumbinary discs.

We fit the relation of the disc radius to age using the Bayesian linear regression method of Kelly (2007), linmix. This method of regression takes into account errors on both axes, as well as intrinsic scatter.

We find a negative trend relating the disc radius to the age of the system with a slope of $-1.3 \pm 1.0$ for the Rim discs. However, we have a large dispersion of $2.7 \pm 2.3 \mathrm{dex}$, indicating that this relationship may not be significant. This high dispersion may be due to the large errors associated with the calculated ages. We confirm this by calculating a Pearson correlation coefficient for the trend. We find a slightly negative relationship with an R-value of -0.22 . We measure a p-value of 0.24 , indicating that this slight negative trend may not be statistically significant.

The same analysis is performed for the Ring discs and we find a slightly negative trend with a slope of $-2.1 \pm 4.0$ and a high dispersion of $3.2 \pm 3.0 \mathrm{dex}$. This negative correlation, however, has an R-value of -0.33 , with a p-value of 0.21 , thus indicating that this negative trend is not statistically significant. Therefore, we conclude that there is no correlation between the radial extent of the ring discs and the age of the system.

The results presented here agree with the work done by van der Marel et al. (2019) who showed that there was no correlation between the radius of the disc and the age of the system.

\subsubsection{Horseshoe}

Fig. C3 shows the Horseshoe discs arranged according to age. There is a wide range of ages for the protoplanetary discs that feature a Horseshoe morphology. AB Auriga, the youngest star, has an age of $3.0 \pm 0.5 \mathrm{Myr}$, while the oldest disc, SAO 206462, is $14.0 \pm 5.5 \mathrm{Myr}$ old. Therefore, the horseshoe-like morphology may be caused by a mechanism that occurs in the later evolutionary stage of a protoplanetary disc. However, due to our small sample and a lack of young intermediate stars observed with ALMA at a high resolution, we cannot make any definitive conclusions about this.

There appears to be no variation in the morphological structure of the discs as they get older. It can be seen that the radius of the disc is not correlated with the age of the star. The radii of the discs surrounding the intermediate-mass stars AB Auriga, HD142527, $\mathrm{HD} 34282$, and SAO206462 are $163 \pm 16,186 \pm 27,187 \pm 28$, and $120 \pm 62$, respectively. However, our sample only features four protoplanetary discs with a horseshoe morphology, therefore any conclusions may be due to small number statistics.

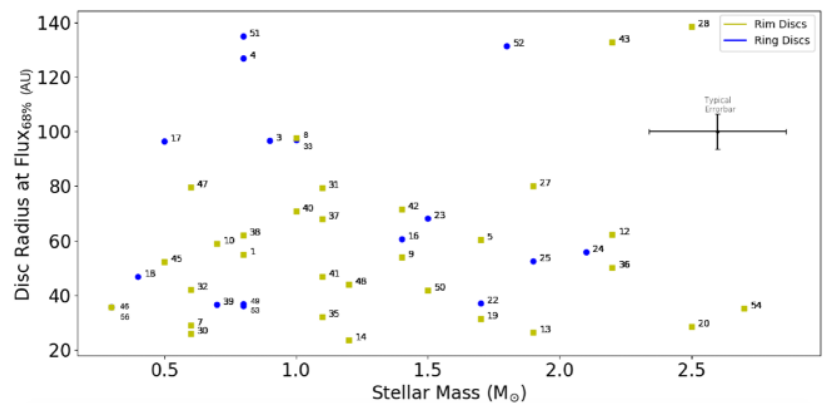

Figure 8. The radii of the protoplanetary discs containing 68 per cent of the total flux against the stellar mass. The yellow markers indicate the Rim discs and the blue markers indicate the Ring discs.

We do not make use of the derived radii for the Horseshoe and Spiral discs as we only have four discs in each category; an insufficient sample to derive meaningful relationships.

\subsection{Structure versus mass}

We have also investigated whether the substructures seen in a protoplanetary disc are dependant on stellar mass. The stellar masses have been calculated in Section 4.1 and can be found in Table 1 . Figs C5, C6, C7, and C8 can be found in Appendix C and show the Rim, Ring, Horseshoe, and Spiral discs ordered according to stellar mass. The images of the low-mass stars have been scaled to $500 \times 500 \mathrm{au}$, while the stars with a $\dagger$ symbol have been scaled to $1000 \times 1000 \mathrm{au}$. This again was done to enable us to compare the spatial extents of the discs.

By looking at Figs C5, C6, C7, and C8, we can see that there is no correlation between the mass of the host star and the outer radius of the protoplanetary disc. This is true for both the lowand intermediate-mass stars of our sample. Our sample covers host stars with a wide range of stellar masses: from $\sim 0.3$ to $>3.0 \mathrm{M}_{\odot}$ for the stars surrounded by a rim disc, $\sim 0.3$ to $4.0 \mathrm{M}_{\odot}$ for the Ring protoplanetary discs, $\sim 1.5$ to $2.7 M_{\odot}$ for the horseshoe-shaped protoplanetary discs, and $\sim 0.5$ to $1.0 \mathrm{M}_{\odot}$ for the stars surrounded by a spiral-shaped protoplanetary disc.

We confirm this by following the same method adopted when studying the relation between the radius of the disc and the age of the system in Section 6.2 above. A graph relating the mass of the star and the radius of the disc can be seen in Fig. 8. The values for the derived disc radii and stellar masses can be found in Tables 2 and 1 , respectively. We have not examined trends for the Horseshoe and Spiral discs as we only have a small sample of discs. We have also omitted the circumbinary discs.

We find positive trends relating the mass of the star to the outer radius of the disc for both the Rim and Ring discs. However, the large errors associated with the determination of the stellar mass make these trends uncertain. The Rim discs have a slope of $5.3 \pm 10.9$ with a large dispersion of $2.8 \pm 2.3$ dex. We confirm that this is a weak relationship by calculating a correlation coefficient for the trend. We find an R-value of 0.24 with a p-value of 0.21 for the trend relating the stellar mass of the system to the radius of the disc. The trend relating the radius to the stellar mass of the Ring discs has a large dispersion of $3.2 \pm 3.0$ dex. We calculate a correlation coefficient of 0.03 for this trend with a p-value of 0.92 . This is strong evidence for there being no correlation between the outer radius of the Ring discs and the stellar mass. This is in agreement with the result found by van der Marel et al. (2019). 
Table 3. The discs observed by ALMA using a high enough resolution and sensitivity to justify there being undetected substructure. See the text for further details. The IDs correspond to the IDs of the discs listed in Table A1. Distances have been obtained from Gaia DR2 unless otherwise stated (Gaia Collaboration 2018).

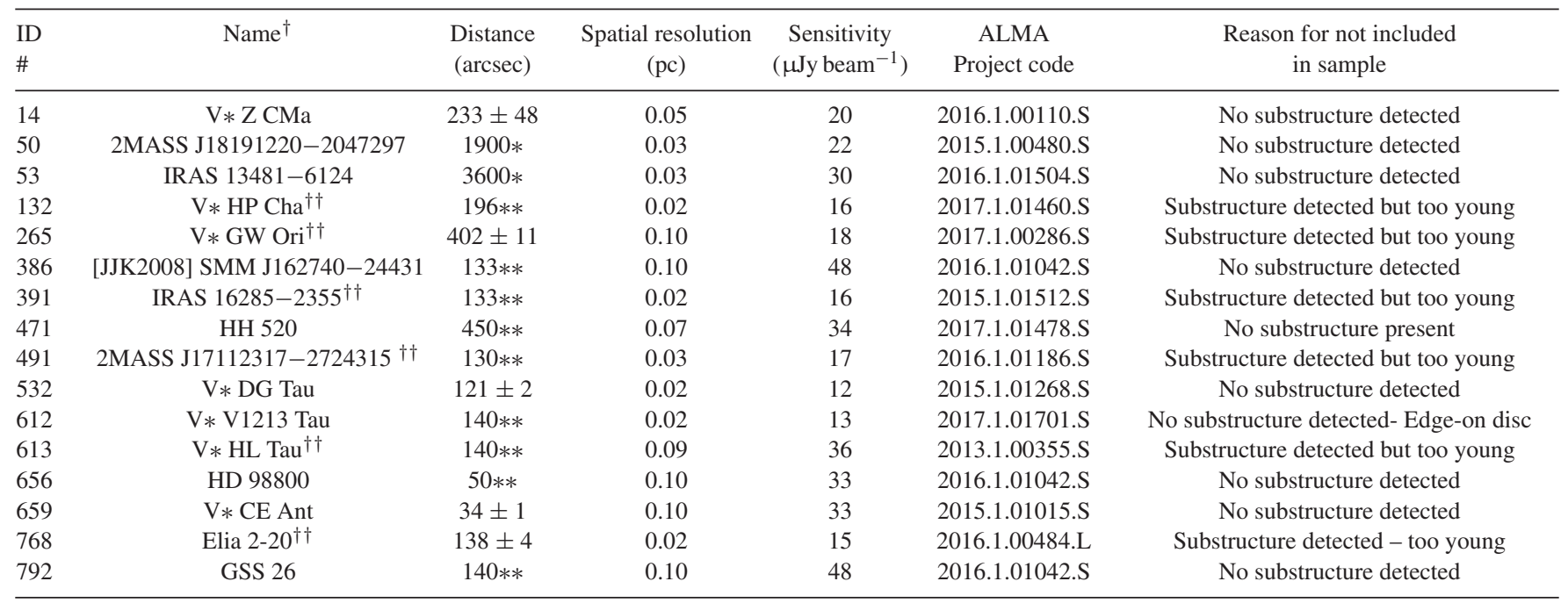

Notes.

${ }^{\dagger}$ The name of each object given here can be queried in data bases such as SIMBAD. An alternative name for each object may have been used on the ALMA Archive, in which case the project code should also be used to find the object.

${ }^{\dagger}$ These observations show substructure but were discarded from our sample due to our selection criteria (see Section 2). These discs all have ages less than $0.5 \mathrm{Myr}$ - see the text for further details.

*These sources have no associated Gaia distances and their distance was obtained from literature measurements - see the text for details.

**These sources have no associated Gaia distance, nor individual distance estimate. Therefore, we approximate the distance by using the distance to its association.

\section{DISCUSSION}

\subsection{The detectability of substructures}

We previously introduced the resolution bias of our sample in Section 3.1. Our finding that $\sim 27$ per cent of discs show substructure when observed with a resolution limit of 0.22 arcsec agrees with Cieza et al. (2019). These authors find that 20 per cent of their discs show substructure for the same resolution limit.

Furthermore, we were able to show that just less than half of the discs observed by ALMA show substructure when observed with a resolution limit of $\approx 0.12$ arcsec, in agreement with Long et al. (2019). Higher resolution observations of protoplanetary discs are needed in order to bring the frequency of discs showing substructure in line with the results of the DSHARP project, where 60 per cent of discs showed substructure when observed with very high resolutions of $\approx 0.035$ arcsec.

Similarly, only 25 percent of discs observed by ALMA show substructure when observed with a sensitivity limit of $50 \mu \mathrm{Jy}_{\text {beam }}{ }^{-1}$ or greater. This increases to 35 per cent when the sensitivity increases to $20 \mu \mathrm{Jy}_{\text {beam }}{ }^{-1}$ or greater. Both Long et al. (2019) and Andrews et al. (2018b) were able to find a higher proportion of discs to show substructure when using these sensitivity limits $(\leq 50$ percent and 100 per cent, respectively). Therefore, the fraction of discs showing substructure here is smaller than is expected for these sensitivity limits. This could imply that other factors, such as the resolution, may be affecting our ability to detect substructure in the discs even though our sensitivities are high enough.

We look at the 738 discs that do not show substructure - or showed substructure but were not included during our sample selection (see Section 2) - to determine the number of discs that are bright and extended enough to justify substructure that is undetected. We use our previous resolution and sensitivity limits of $0.1 \mathrm{arcsec}$ and
$50 \mu \mathrm{Jy}$ beam $^{-1}$, where Long et al. (2019) were able to show that just under half of the discs in their sample show substructure. We find that 98 discs meet the sensitivity limit, while 30 discs meet the resolution limit. Of these objects, 16 discs are both bright and extended enough within our limits to show substructure. These discs are shown below in Table 3.

Six of these 16 discs showed substructure, but were removed from our sample due to having ages less than 0.5 Myr. These objects are V* HP Cha, V* GW Ori, IRAS 16285-2355, 2MASS J17112317-2724315, V* HL Tau, and Elias 2-20 (Welch et al. 2000; Brooke et al. 2007; Fang et al. 2014; Soderblom et al. 2014; Czekala et al. 2017; Segura-Cox et al. 2020). Furthermore, two discs were removed from our sample due to their large distances. Objects 2MASS J18191220-2047297 and IRAS 13481-6124 have distances of 1900 and 3600 pc, respectively (Busfield et al. 2006; Maud et al. 2015). Therefore, currently, no substructure can be detected in their discs regardless of how high the resolution and sensitivity are. The object $\mathrm{V} * \mathrm{~V} 1213$ Tau is an edge-on protoplanetary disc, therefore we are unable to detect substructure in the disc (Burrows et al. 1996; Stapelfeldt et al. 1999).

We show the continuum product images of the remaining seven discs that have been observed with a sufficiently high resolution and sensitivity to warrant substructure in Fig. 9. Reobservation of these discs at higher resolution and sensitivities may result in detection of substructure.

The resolution and sensitivity used to observe protoplanetary discs may also affect the type of substructure that is detected. We plot the resolution of the observations used to detect our sample against the sensitivities in Fig. 10. We find that the majority of discs featuring rings are detected when the sensitivity is below $\sim 60$ $\mu \mathrm{Jy} \mathrm{beam}^{-1}$, regardless of the resolution used. Rim discs, on the other hand, span a wide resolution and sensitivity range. However, below $20 \mu \mathrm{Jy}$ beam $^{-1}$, five ring discs are detected compared to 

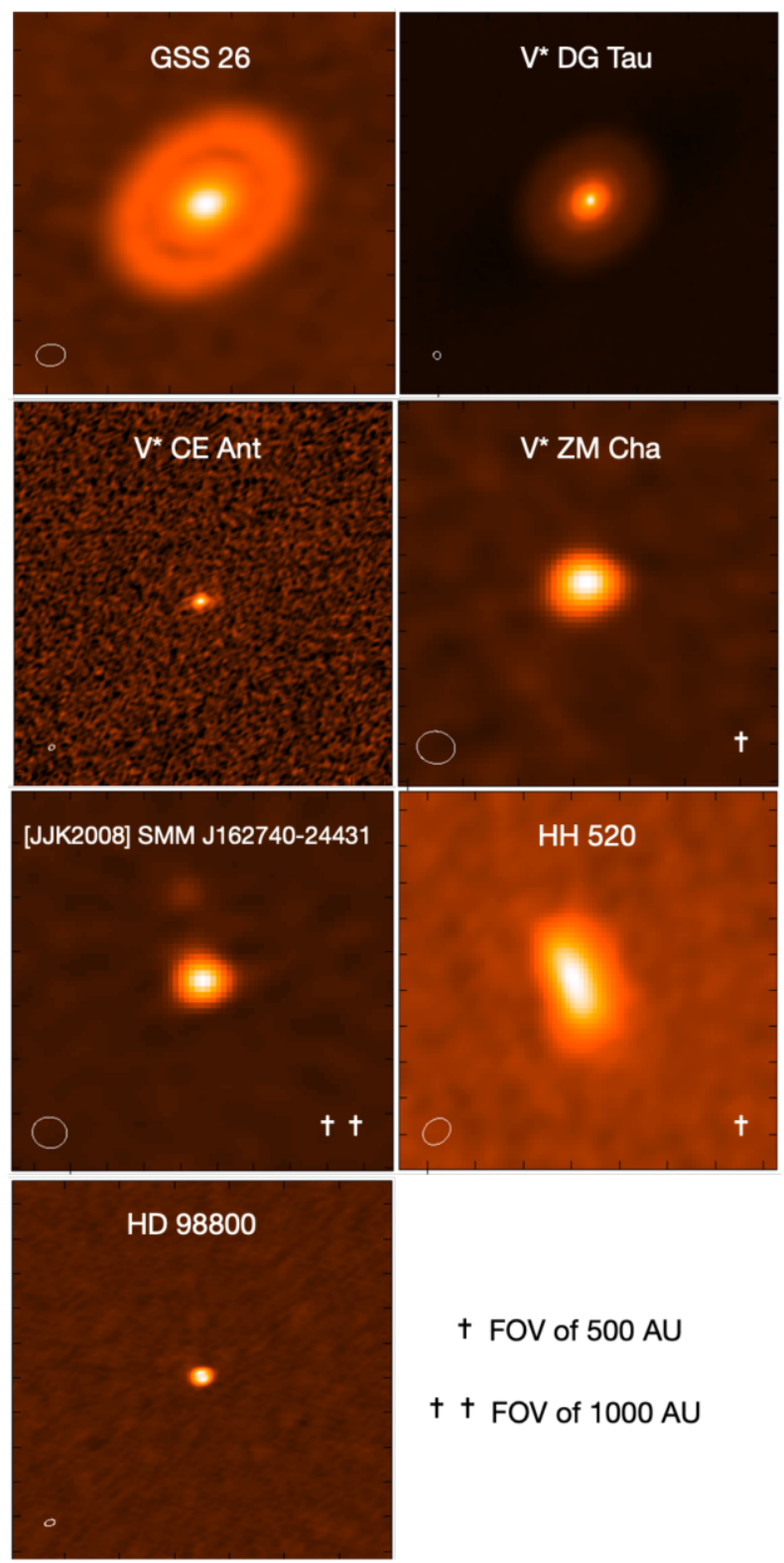

\section{+ FOV of $500 \mathrm{AU}$}

\section{+ t FOV of $1000 \mathrm{AU}$}

Figure 9. The protoplanetary discs observed with a high enough resolution and sensitivity to hint at unresolved substructure. The images show a field of view of $250 \times 250$ au unless otherwise stated. The beam of the observations are shown in the bottom left corner.

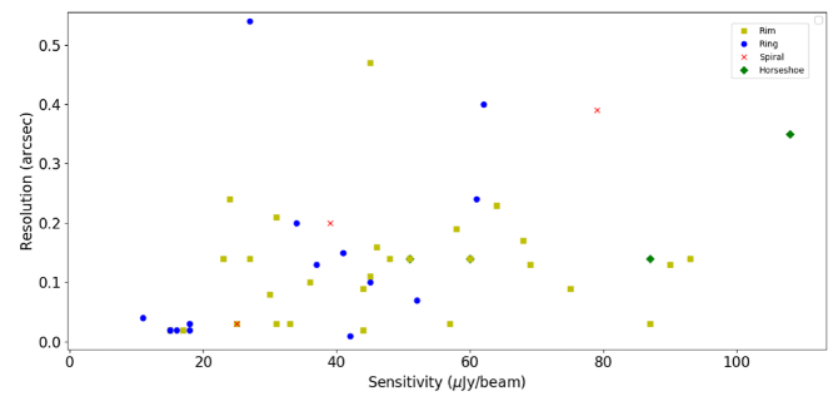

Figure 10. A plot of the angular resolutions and sensitivities of the observations of the discs in our sample. We have omitted the four protoplanetary discs with sensitivities greater than $120 \mu \mathrm{Jy}_{\text {beam }^{-1}}$. only one rim disc. Thus, additionally, supporting the notion that increasing the resolution and sensitivity of observations may cause discs to be reclassified from Rim to Rings. Therefore, although angular resolution may play a vital role in detecting substructure in protoplanetary discs (as we have shown in Section 3.1), the sensitivity of the observations is key in determining the type of substructure present in the disc.

\subsubsection{The observability of rims}

32 discs in our sample have been classified as having a Rim; a single ring of dust surrounding a large cavity devoid of dust and/or gas. It is clear from Fig. 4 that the hosts stars that are surrounded by a Rim disc cover both stellar temperature and luminosity quite uniformly. There are somewhat more Rim discs around low-mass stars than around intermediate-mass stars (24 low-mass stars and eight intermediatemass stars). However, due to the nature of our classification scheme, all four of the high-mass Horseshoe discs could also be classified as Rim discs. Therefore, a single ring of emission on the edge of a large cavity appears to be a very common feature seen in protoplanetary discs regardless of temperature or luminosity.

There are numerous ways to form a rim of dust around a star and this may explain why they are a frequent substructure seen in protoplanetary discs. A planet present in a disc would cause a pressure maximum to form. As dust radially migrates inwards from the outer regions of the disc it hits this pressure maximum. This would cause a pile-up of dust grains to form in a ring around the orbit of the forming protoplanet (Paardekooper \& Mellema 2006; Fouchet et al.2007).

In order to detect if a rim has been formed due to a planetary companion, observations of CO isotopologues can be made. Gas gaps have previously been shown to have been carved out by a planetary or substellar companion (Bruderer et al. 2014; van der Marel et al. 2015, 2016b; Dong et al. 2017; Boehler et al. 2017, 2018) In recent work, Ubeira Gabellini et al. (2019) were able to show that depressions in both continuum and $\mathrm{CO}$ isotopologue ring maps could be caused by an embedded planet within the disc.

A rim can also form in the disc due to photoevaporation. UV radiation from the central star can heat up the surface of the disc. This can cause the dust to evaporate and eventually leave the disc as a photoevaporative wind, thus forming a cavity (Hollenbach et al. 1994; Hardy et al. 2015) A rim can then be seen at the outer edge of the cavity. A rim, as the outer edge of a cavity, can also form in a protoplanetary disc due to condensation fronts (Zhang et al. 2015), aggregate sintering (Okuzumi et al. 2016), and the inner edge of a dead zone (Flock et al. 2015; Béthune, Lesur \& Ferreira 2016).

The populous nature of rings, however, may be due to a selection bias. Transition discs have explicitly been targeted by ALMA, both in individual projects as well as part of surveys (e.g. DSHARP, Andrews et al. 2018b). This is because rim discs are easily detected, even at the lower resolutions and sensitivities. More complex substructure, such as spiral and horseshoes, may only be detected at higher resolutions and sensitivities.

This resolution dependence has recently been demonstrated in ALMA observations of LkCa15 by Facchini et al. (2020). In previous works, and here, it has been classified as a rim surrounding a cavity. However, ALMA observations with a resolution $\sim 0.04$ arcsec of were able to resolve multiple rings in the disc. In addition to this, Francis \& van der Marel (2020) were able to show that the cavities of transition discs are often not void of dust. Therefore, there may be unresolved dust rings in some of the discs classified as being Rim discs in this work. Further high-resolution observations may reveal that Rings are the most populous substructure seen in protoplanetary 
discs and not a single rim of emission. Likewise, high-resolution observations would also increase the number of discs that are known to have resolvable substructure.

\subsubsection{Observing horseshoes}

Recent work by van der Marel et al. (2021) has shown that the diversity in asymmetric and non-asymmetric dust structures in protoplanetary discs is linked to the local gas surface density at the location of pressure bumps. Asymmetric structures such as horseshoes may only be detected if the local gas surface density is sufficiently low. The upcoming ngVLA may be able to trace the dust at these low gas density locations using centimetre wavelengths, thus increasing the population of discs showing asymmetric structures. The increased resolution capabilities of the ngVLA may also resolve new substructures and increase the population of discs showing substructures to greater than 60 per cent, as shown by the DSHARP project (Andrews et al. 2018b).

\subsubsection{The observability of rings}

The low-mass stars that feature protoplanetary rings have a wide range of ages; from 0.6 Myr for RU Lup to 10.3 Myr for HD 142666. The spread of ages imply that rings can form very early in the evolution of the disc and are long-lasting substructures or that they can form at a range of evolutionary stages.

Recent observations of HL Tau, Elias 2-24, and GY 91 revealed protoplanetary discs with multiple rings and gaps (ALMA Partnership et al. 2015; Dipierro et al. 2018; Sheehan \& Eisner 2018). These young stellar objects all have ages less than $1 \mathrm{Myr}$. Therefore, planet formation is thought to begin very early in the evolution of the disc. As the protoplanets grow in mass and size, they are able to carve gaps in the dust and gas of the protoplanetary disc forming rings. Mamajek (2009) showed that planet formation could end between 2 and $3 \mathrm{Myr}$ as half of the protoplanetary discs in their sample disappeared by this time. The majority of the low-mass discs in our sample have derived ages greater than 2 Myr (in part due to the removal of young, embedded discs from the sample), meaning that it is possible for their gaps and rings to have been formed by planets. In order to confirm this, similarly to rim discs, additional $\mathrm{CO}$ isotopologue observations could be carried out to determine the origin of the dust rings. However, the gas gaps in ring discs are harder to constrain, as discussed by Isella et al. (2016).

Other mechanisms, such as the accumulation and growth of material at various ice lines (Zhang et al. 2015), may be responsible for the ringed substructure. However, this method was recently put into question by Huang et al. (2018) and van der Marel et al. (2019) who showed that the gap radii in ringed protoplanetary discs do not correspond with common ice lines. The analysis conducted in this work cannot determine the precise origins of the protoplanetary rings seen.

\subsection{Comparisons to scattered light observations}

Observations of protoplanetary discs have previously been made at a number of different wavelengths, including optical, near-infrared, and sub-mm. These different wavelengths probe different regions of the protoplanetary disc. Observations at near-infrared wavelengths trace the smallest dust grains (typically $\mu \mathrm{m}$-sized) in the innermost parts and surface of the protoplanetary disc. At these wavelengths the dust is optically thick. As the observing wavelength approaches $\mathrm{mm} / \mathrm{sub}-\mathrm{mm}$, the dust in the mid-plane of the disc begins to become
Table 4. The discs studied in both this work at mm-wavelengths and by Garufi et al. (2018) in scattered light.

\begin{tabular}{|c|c|c|c|}
\hline $\begin{array}{l}\text { ID } \\
\#\end{array}$ & Name & $\begin{array}{l}\text { Classification at } \\
\text { mm wavelength }\end{array}$ & $\begin{array}{c}\text { Classification in } \\
\text { scattered light }\end{array}$ \\
\hline 2 & AB Auriga & Horseshoe & Spiral \\
\hline 3 & AS 209 & Rings & Rings \\
\hline 5 & CQ Tau & Rim & Spiral \\
\hline 9 & DoAr44 & Rim & Rim \\
\hline 12 & $\mathrm{EM} * \mathrm{SR} 21 \mathrm{~A}$ & Rim & Rings \\
\hline 15 & GG Tau & Rim & Rim \\
\hline 16 & GM Auriga & Rings & Rim \\
\hline 19 & HD 100453 & Rim & Spiral \\
\hline 20 & HD 100546 & Rim & Spiral \\
\hline 21 & HD 142527 & Horseshoe & Spiral \\
\hline 23 & HD 143006 & Rings & Rings \\
\hline 24 & HD 163296 & Rings & Rings \\
\hline 25 & HD 169142 & Rings & Rings \\
\hline 26 & HD 34282 & Horseshoe & Spiral \\
\hline 27 & HD 36112 & Rim & Spiral \\
\hline 28 & HD 97048 & Rim & Rings \\
\hline 29 & IM Lup & Spiral & Rings \\
\hline 31 & LkCa15 & Rim & Rim \\
\hline 33 & $\mathrm{~J} 16042165-2130284$ & Rim & Rim \\
\hline 34 & $\mathrm{~J} 16152023-3255051$ & Spiral & Rings \\
\hline 37 & PDS 70 & Rim & Rim \\
\hline 41 & RXJ1852.3-3700 & Rim & Rings \\
\hline 44 & SAO 206462 & Horseshoe & Spiral \\
\hline 47 & SZ 91 & Rim & Rim \\
\hline 49 & TW Hya & Rings & Rings \\
\hline 50 & UX Tau & Rim & Rim \\
\hline 52 & V1247 Ori & Rings & Spiral \\
\hline 53 & V4046 Sgr & Rings & Rings \\
\hline
\end{tabular}

optically thin. The largest dust grains lie in the mid-plane of the disc and this is where the majority of the disc mass lies.

Substructures can be seen in protoplanetary discs in both $\mathrm{mm}$ and polarized scattered light observations. Garufi et al. (2018) studied the morphology of 58 protoplanetary discs in scattered light and classified them into the following categories: Rim, Rings, Spirals, Faint, Giant, Inclined, and Small. Our sample has 36 protoplanetary discs in common with the sample studied by Garufi et al. (2018). Five discs have been classified as Spiral, five discs as Giant, five as Faint, six as Rim, 11 as Rings, three as Inclined, and one as small.

We have discarded the Faint, Giant, Small, and Inclined categories as they are not morphological structures and have reclassified these discs according to our classification. The scattered light observation of RU Lup, Sz111, CI Tau, MWC 480, and GW Lup were classified as Faint in Garufi et al. (2018), while HD142666, T Cha, and RY Lup were classified as Inclined and CS Cha as Small. Since there is no clear substructure in the these discs and they do not fit in any of our classification categories, we have discarded them from the common sample, leaving 28 discs for comparison. Following the characteristics outlined in Section 5, three of the five discs classified as Giant by Garufi et al. (2018) have been classified as Spiral discs (AB Auriga, HD142527, and HD100546). These three discs are all intermediate-mass stars. The remaining two low-mass stars have been classified as Rim discs (GG Tau A and GM Auriga). The common sample and their classifications, including the newly reclassified Giant discs, can be found in Table 4 .

Fig. 11 compares the morphology seen in scattered light with the morphology seen in sub-mm for the 27 protoplanetary discs. The 


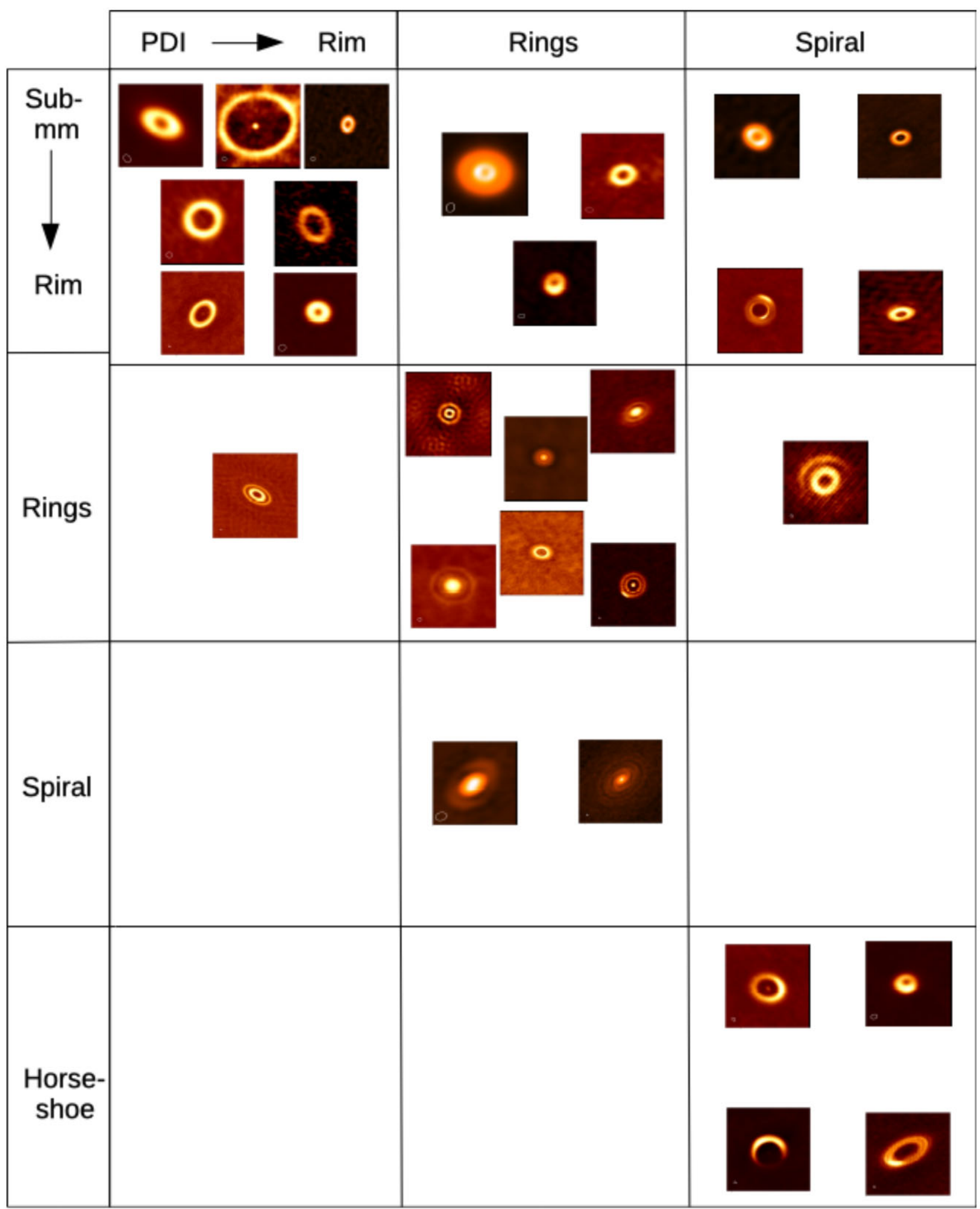

Figure 11. A comparison between the morphology seen in protoplanetary discs at sub-mm continuum and near-IR polarized differential imaging. The columns show the morphology seen in scattered light and the rows show the morphology seen in sub-mm continuum. 
columns indicate the morphology assigned by Garufi et al. (2018) and the rows indicate the morphologies assigned in this work.

All of the discs showing a Horseshoe-shaped morphology in submm continuum emission have a Spiral morphology in scattered light. The disc surrounding SAO206462 was shown to have a mass of $\sim 0.002 \mathrm{M}_{\odot}$ (Pérez et al. 2014), while the disc masses of AB Auriga, HD142527, and HD100546 have been shown to be $0.01 \mathrm{M}_{\odot}$ (Tang et al. 2012), $0.1 \mathrm{M}_{\odot}$ (Perez et al. 2015), and $\sim 0.03 \mathrm{M}_{\odot}$ (Kama et al. 2016), respectively. The wide range of disc masses may indicate that disc mass plays little role in the type of morphology seen in discs in both sub-mm and scattered light.

Spiral substructure seen in scattered light observations has previously been seen in a number of protoplanetary discs (Grady et al. 2001, 2013; Fukagawa et al. 2004; Casassus et al. 2012; Muto et al. 2012; Wagner et al. 2015; Akiyama et al. 2016; Ohta et al. 2016; Canovas et al. 2018). The submillimetre counterpart observations to these discs, however, show large cavities and axisymmetric substructure (Isella et al. 2013; van der Marel et al. 2013; Ansdell et al. 2016; Kraus et al. 2017; Tang et al. 2017; Cazzoletti et al. 2018; Dong et al. 2018; Ohashi et al. 2018; Pineda et al. 2019). The mm images shown here of IM Lup and J16152023 both feature a spiral-like morphology, however rings were seen in scattered light observations rather than large cavities and axisymmetric substructures. This agrees with the results found by Avenhaus et al. (2018) and Huang et al. (2018) for these two discs.

The nine discs shown here with a spiral-like morphology in scattered light were found to be relatively old (Garufi et al. 2018). The host stars had ages between $\approx 3$ and $\approx 12 \mathrm{Myr}$ and are at the very late stage of their PMS lifetime. Also, the lack of young stars featuring a Spiral disc led to the conclusion that the formation of spiral arms in $\mu \mathrm{m}$-sized dust discs may only occur in the latter phases of their PMS lifetime. This contrasts with the results found in this work. The four Spiral discs in this work are at an early stage of their PMS lifetime and have ages $\leq 6 \mathrm{Myr}$. Therefore, the mechanism responsible for forming spiral arms in the sub-mm dust of a protoplanetary disc happens during the earliest stages of its evolution around low-mass stars. This discrepancy may suggest that there are different mechanisms forming the spiral arms seen in scattered light and those seen in the sub-mm.

14 discs have been classified as Rim discs in the sub-mm, 10 of them being low-mass stars and four being intermediate-mass stars. Seven low-mass stars have been shown to have Rims in both sub-mm and scattered light. EM $*$ SR21 and HD 97048 are both intermediate-mass stars that show a Rim in sub-mm but have a ringed protoplanetary disc in scattered light. Both $\mathrm{EM} * \mathrm{SR} 21$ and HD97048 have relatively young ages. Recent work by Muro-Arena et al. (2020) and van der Marel et al. (2021) has shown that the structure within these discs may be more complex and it may be possible for multiple rings to be present. Observations at a higher resolution would need to be conducted in order to confirm the structure within the disc.

All the discs in our sample (56 discs) show some sort of cavity or gap. 65 percent of the discs studied by Garufi et al. (2018) showed some sort of cavity in scattered light indicating that they are a common occurrence in both sub-mm and scattered light. The discs that showed a cavity in sub-mm and not scattered light were classified by Garufi et al. (2018) as either Faint or Inclined. The lack of a cavity may contribute to the faintness of the discs in scattered light, while the absence of a cavity in the Inclined discs may be an observational bias (Garufi et al. 2018). The high number of discs that exhibit a cavity in both scattered light and sub-mm shows that cavities are a common feature found in protoplanetary discs across a range of star type and evolutionary stage. However, it should be noted that there may be a bias in both samples. The sample by Garufi et al. (2018) may contain many transition discs as they are bright due to their cavity wall. Likewise, the sample presented here may be biased towards transition discs as they are easiest to find at low resolution.

Eight discs classified as Rings in sub-mm have been studied in scattered light, six of which surround low-mass stars and two surrounding intermediate stars. Rings in scatted light are seen in six of the protoplanetary discs as well as in the sub-mm. The two systems that do not show rings in scattered light are GM Auriga and V1247 Ori, both low-mass stars. The discs around these stars were shown to have a Rim and Spiral morphology, respectively. The rings seen in protoplanetary discs in scattered light and in the sub-mm can form with similar mechanisms, such as the interaction of the disc with a companion (Lin \& Papaloizou 1979; Kley \& Nelson 2012; Pinilla, Benisty \& Birnstiel 2012; de Juan Ovelar et al. 2013).

\section{CONCLUSIONS}

We have studied the morphology of the substructure seen in protoplanetary discs. We have classified 56 discs with visible structure in the ALMA Archive cycles 0 to 6 . These discs, observed in the sub-mm continuum, were placed into four categories: Rim, Ring, Horseshoe, and Spiral. By calculating the age of the host stars we are able to study the evolution of the substructures seen in protoplanetary discs over a range of ages. The sub-mm images studied in this work were then compared to scattered light observations of protoplanetary discs studied by Garufi et al. (2018). We have reached the following conclusions in this work:

(i) We find that 27 percent of 798 discs observed with ALMA during cycles $0-5$ show substructure when observed with a moderate resolution of at least $\sim 0.22$ arcsec. Using a higher resolution of $\sim 0.1$ arcsec, 42 per cent of the discs observed show clear substructure. The fraction of discs showing substructure increases to 60 per cent when observed with a very high resolution of $\$ 0.04$ arcsec. Therefore, many of the discs observed with ALMA thus far may have substructure that remains unresolved due to the relatively low resolutions used.

(ii) When looking at the 798 discs observed with ALMA during cycles $0-5$, we find that 31 (or 25 per cent) show substructure when observed with a moderate sensitivity of $50 \mu \mathrm{Jy}$ beam $^{-1}$. A higher sensitivity of $20 \mu \mathrm{Jy}$ beam $^{-1}$ results in 35 percent of the discs showing substructure. This low proportion indicates that many of the discs observed by ALMA thus far may feature substructure that remains undetected due to relatively low sensitivities used.

(iii) We have shown that angular resolution is key in detecting substructures within protoplanetary discs, with sensitivity playing a vital role in determining the type of substructure present in the disc.

(iv) The protoplanetary discs studied here show substructure surrounds Class II young stellar objects with a range of ages. This is representative of the full sample of discs observed by ALMA thus far, where the majority of the systems observed have been Class II young stellar objects. The discs studied here, however, were observed with much higher resolutions than the majority of the discs in the ALMA Archive. Therefore, they are not representative of many discs which were observed at significantly lower resolutions.

(v) In our sample the most populous substructure seen is a rim of sub-mm dust surrounding a large cavity. A rim of some sort is found for over half of the discs in our sample and they appear around stars with a range of ages, temperatures, and luminosities. This type of substructure, however, is the easiest to detect and have been explicitly 
targeted by ALMA. None the less, it confirms that cavities and gaps are a common feature in protoplanetary discs.

(vi) The second most common substructure seen in our protoplanetary discs is a ringed disc. As the majority of the discs observed with ALMA thus far were not observed using sufficiently high resolution, a Ring disc may be the most common substructure seen in protoplanetary discs.

(vii) A distinctive horseshoe-shaped protoplanetary disc has only been seen in a few systems. These systems contain relatively old, intermediate-mass stars.

(viii) All discs showing a horseshoe morphology in the sub-mm continuum images show a spiral-like morphology in scattered light.

(ix) Low-mass stars that have been classified as Rim discs in the sub-mm have also been classified as Rim in scattered light. The occurrence of a single rim of emission around a large cavity seems to be a common substructure found in both scattered light and sub-mm continuum emission.

(x) The substructures seen in ALMA images of protoplanetary discs mostly do not seem to follow an evolutionary sequence nor do they depend on the mass of the star.

In future work we will compare the images with theoretical predictions.

\section{ACKNOWLEDGEMENTS}

This paper makes use of the following ALMA data: ADS/JAO.ALMA\#2011.0.00724.S, ADS/JAO.ALMA\#2012.1.00158.S, ADS/JAO.ALMA\#2012.1.00182.S, ADS/JAO.ALMA\#2012.1.00303.S, ADS/JAO.ALMA\#2012.1.00631.S, ADS/JAO.ALMA\#2012.1.00799.S, ADS/JAO.ALMA\#2012.1.00870.S, ADS/JAO.ALMA\#2013.1.00157.S, ADS/JAO.ALMA\#2013.1.00091.S, ADS/JAO.ALMA\#2013.1.00105.S ADS/JAO.ALMA\#2013.1.00220.S, ADS/JAO.ALMA\#2013.1.00226.S, ADS/JAO.ALMA\#2013.1.00498.S, ADS/JAO.ALMA\#2013.1.00658.S, ADS/JAO.ALMA\#2013.1.00663.S, ADS/JAO.ALMA\#2013.1.00355.S, ADS/JAO.ALMA\#2015.1.00888.S, ADS/JAO.ALMA\#2015.1.00486.S, ADS/JAO.ALMA\#2015.1.00806.S, ADS/JAO.ALMA\#2015.1.00847.S, ADS/JAO.ALMA\#2015.1.00964.S, ADS/JAO.ALMA\#2015.1.00986.S, ADS/JAO.ALMA\#2015.1.01017.S, ADS/JAO.ALMA\#2015.1.01083.S, ADS/JAO.ALMA\#2015.1.01301.S, ADS/JAO.ALMA\#2015.1.00480.S, ADS/JAO.ALMA\#2015.1.01512.S, ADS/JAO.ALMA\#2015.1.01268.S, ADS/JAO.ALMA\#2015.1.01015.S, ADS/JAO.ALMA\#2016.1.00484.L, ADS/JAO.ALMA\#2016.1.00826.S, ADS/JAO.ALMA\#2016.1.01042.S, ADS/JAO.ALMA\#2016.1.01164.S, ADS/JAO.ALMA\#2016.1.01239.S, ADS/JAO.ALMA\#2016.1.00110.S,
ADS/JAO.ALMA\#2016.1.01504.S, ADS/JAO.ALMA\#2016.1.01042.S, ADS/JAO.ALMA\#2016.1.01186.S, ADS/JAO.ALMA\#2017.1.00449.S, ADS/JAO.ALMA\#2017.1.00520.S, ADS/JAO.ALMA\#2017.1.00969.S, ADS/JAO.ALMA\#2017.1.01151.S, ADS/JAO.ALMA\#2017.1.01167.S, ADS/JAO.ALMA\#2017.1.01424.S, ADS/JAO.ALMA\#2017.1.01460.S, ADS/JAO.ALMA\#2017.A.00006.S and ADS/JAO.ALMA\#2017.A.00014.S, ADS/JAO.ALMA\#2017.1.00286.S, ADS/JAO.ALMA\#2017.1.01478.S, ADS/JAO.ALMA\#2017.1.01701.S. ALMA is a partnership of ESO (representing its member states), NSF (USA) and NINS (Japan), together with NRC (Canada), MOST and ASIAA (Taiwan), and KASI (Republic of Korea), in cooperation with the Republic of Chile. The Joint ALMA Observatory is operated by ESO, AUI/NRAO, and NAOJ.

\section{DATA AVAILABILITY}

The data underlying this article are available in the ALMA Archive, at https://almascience.nrao.edu/aq/. The project codes for the data have been provided in the Acknowledgements section above.

\section{REFERENCES}

Agra-Amboage V., Dougados C., Cabrit S., Garcia P. J. V., Ferruit P., 2009, A\&A, 493, 1029

Akeson R. L., Jensen E. L. N., 2014, ApJ, 784, 62

Akeson R. L., Jensen E. L. N., Carpenter J., Ricci L., Laos S., Nogueira N. F., Suen-Lewis E. M., 2019, ApJ, 872, 158

Akiyama E. et al., 2016, AJ, 152, 222

Alcalá J. M. et al., 2017, A\&A, 600, A20

ALMA Partnership et al., 2015, ApJ, 808, L3

Andrews S. M. et al., 2018b, ApJ, 869, L41

Andrews S. M., Terrell M., Tripathi A., Ansdell M., Williams J. P., Wilner D. J., 2018a, ApJ, 865, 157

Ansdell M. et al., 2016, ApJ, 828, 46

Ansdell M. et al., 2018, ApJ, 859, 21

Ansdell M., Williams J. P., Manara C. F., Miotello A., Facchini S., van der Marel N., Testi L., van Dishoeck E. F., 2017, AJ, 153, 240

Avenhaus H. et al., 2018, ApJ, 863, 44

Baraffe I., Homeier D., Allard F., Chabrier G., 2015, A\&A, 577, A42

Barenfeld S. A., Carpenter J. M., Ricci L., Isella A., 2016, ApJ, 827, 142

Baruteau C. et al., 2014, in Beuther H., Klessen R. S., Dullemond C. P., Henning T., eds, Protostars and Planets VI. University of Arizona Press, Tucson, p. 667

Baruteau C., Zhu Z., 2016, MNRAS, 458, 3927

Bell C. P. M., Mamajek E. E., Naylor T., 2015, MNRAS, 454, 593

Berrilli F., Corciulo G., Ingrosso G., Lorenzetti D., Nisini B., Strafella F., 1992, ApJ, 398, 254

Bertout C., Siess L., Cabrit S., 2007, A\&A, 473, L21

Béthune W., Lesur G., Ferreira J., 2016, A\&A, 589, A87

Beuther H., Churchwell E. B., McKee C. F., Tan J. C., 2007, in Reipurth B., Jewitt D., Keil K., eds, Protostars and Planets V. University of Arizona Press, Tucson, p. 165

Boehler Y. et al., 2018, ApJ, 853, 162

Boehler Y., Weaver E., Isella A., Ricci L., Grady C., Carpenter J., Perez L., 2017, ApJ, 840, 60

Brooke T. Y. et al., 2007, ApJ, 655, 364

Bruderer S., van der Marel N., van Dishoeck E. F., van Kempen T. A., 2014, A\&A, 562, A26 
Burrows C. J. et al., 1996, ApJ, 473, 437

Busfield A. L., Purcell C. R., Hoare M. G., Lumsden S. L., Moore T. J. T., Oudmaijer R. D., 2006, MNRAS, 366, 1096

Caballero J. A., Solano E., 2008, A\&A, 485, 931

Canovas H. et al., 2018, A\&A, 610, A13

Casassus S. et al., 2013, Nature, 493, 191

Casassus S. et al., 2015, ApJ, 812, 126

Casassus S., Perez M. S., Jordán A., Ménard F., Cuadra J., Schreiber M. R., Hales A. S., Ercolano B., 2012, ApJ, 754, L31

Cazzoletti P. et al., 2018, A\&A, 619, A161

Chabrier G., 2003, ApJ, 586, L133

Cieza L. A. et al., 2019, MNRAS, 482, 698

Cox E. G. et al., 2017, ApJ, 851, 83

Czekala I. et al., 2017, ApJ, 851, 132

Da Rio N., Robberto M., Soderblom D. R., Panagia N., Hillenbrand L. A., Palla F., Stassun K. G., 2010, ApJ, 722, 1092

de Juan Ovelar M., Min M., Dominik C., Thalmann C., Pinilla P., Benisty M., Birnstiel T., 2013, A\&A, 560, A111

Di Folco E. et al., 2014, A\&A, 565, L2

Dipierro G. et al., 2018, MNRAS, 475, 5296

Dong R. et al., 2017, ApJ, 836, 201

Dong R. et al., 2018, ApJ, 860, 124

Dong R., Zhu Z., Whitney B., 2015a, ApJ, 809, 93

Dong R., Hall C., Rice K., Chiang E., 2015b, ApJ, 812, L32

Dutrey A., Di Folco E., Beck T., Guilloteau S., 2016, A\&A Rev., 24, 5

Eisner J. A. et al., 2018, ApJ, 860, 77

ESA, 1997, The Hipparcos and Tycho catalogues. Astrometric and photometric star catalogues derived from the ESA Hipparcos Space Astrometry Mission, ESA SP Series vol no: 1200. ESA Publications Division, Noordwijk, Netherlands

Facchini S. et al., 2020, A\&A, 639, A121

Fairlamb J. R., Oudmaijer R. D., Mendigutía I., Ilee J. D., van den Ancker M. E., 2015, MNRAS, 453, 976

Fang M., Sicilia-Aguilar A., Roccatagliata V., Fedele D., Henning T., Eiroa C., Müller A., 2014, A\&A, 570, A118

Fedele D. et al., 2017, A\&A, 600, A72

Fedele D. et al., 2018, A\&A, 610, A24

Flock M., Ruge J. P., Dzyurkevich N., Henning T., Klahr H., Wolf S., 2015, A\&A, 574, A68

Fontani F., Beltrán M. T., Brand J., Cesaroni R., Testi L., Molinari S., Walmsley C. M., 2005, A\&A, 432, 921

Fouchet L., Maddison S. T., Gonzalez J. F., Murray J. R., 2007, A\&A, 474, 1037

Francis L., van der Marel N., 2020, ApJ, 892, 111

Fukagawa M. et al., 2004, ApJ, 605, L53

Fukagawa M., Tamura M., Itoh Y., Kudo T., Imaeda Y., Oasa Y., Hayashi S. S., Hayashi M., 2006, ApJ, 636, L153

Gaia Collaboration, 2016, A\&A, 595, A2

Gaia Collaboration, 2018, A\&A, 616, A1

Garufi A. et al., 2018, A\&A, 620, A94

Garufi A. et al., 2019, A\&A, 628, A68

Gonzalez J. F., Laibe G., Maddison S. T., 2017, MNRAS, 467, 1984

Grady C. A. et al., 2001, AJ, 122, 3396

Grady C. A. et al., 2013, ApJ, 762, 48

Grosso N., Bouvier J., Montmerle T., Fernández M., Grankin K., Zapatero Osorio M. R., 2007, A\&A, 475, 607

Guenther E. W., Esposito M., Mundt R., Covino E., Alcalá J. M., Cusano F., Stecklum B., 2007, A\&A, 467, 1147

Hardy A. et al., 2015, A\&A, 583, A66

Hollenbach D., Johnstone D., Lizano S., Shu F., 1994, ApJ, 428, 654

Huang J. et al., 2018, ApJ, 869, L42

Isella A. et al., 2016, Phys. Rev. Lett., 117, 251101

Isella A., Testi L., Natta A., 2006, A\&A, 451, 951

Isella A., Carpenter J. M., Sargent A. I., 2009, ApJ, 701, 260

Isella A., Pérez L. M., Carpenter J. M., Ricci L., Andrews S., Rosenfeld K., 2013, ApJ, 775, 30

James D. J., Melo C., Santos N. C., Bouvier J., 2006, A\&A, 446, 971

Kama M. et al., 2016, A\&A, 592, A83
Kelly B. C., 2007, ApJ, 665, 1489

Kenyon S. J., Gómez M., Whitney B. A., 2008, in Reipurth B., ed., Handbook of Star Forming Regions, Vol. 4, p. 405

Kley W., Nelson R. P., 2012, ARA\&A, 50, 211

Kraus S. et al., 2013, ApJ, 768, 80

Kraus S. et al., 2017, ApJ, 848, L11

Kraus A. L., Hillenbrand L. A., 2009, ApJ, 704, 531

Kroupa P., 2001, MNRAS, 322, 231

Langlois M. et al., 2018, A\&A, 614, A88

Lin D. N. C., Papaloizou J., 1979, MNRAS, 188, 191

Long F. et al., 2018, ApJ, 869, 17

Long F. et al., 2019, ApJ, 882, 49

Lorén-Aguilar P., Bate M. R., 2016, MNRAS, 457, L54

Luhman K. L., 2007, ApJS, 173, 104

Macías E. et al., 2018, ApJ, 865, 37

Mamajek E. E., 2009, in Usuda T., Tamura M., Ishii M., eds, AIP Conf. Proc. Vol. 1158, Exoplanets and Disks: Their Formation and Diversity. Am. Inst. Phys., New York, p. 3

Mamajek E. E., Bell C. P. M., 2014, MNRAS, 445, 2169

Manoj P., Bhatt H. C., Maheswar G., Muneer S., 2006, ApJ, 653, 657

Maud L. T., Moore T. J. T., Lumsden S. L., Mottram J. C., Urquhart J. S., Hoare M. G., 2015, MNRAS, 453, 645

Mendigutía I., Fairlamb J., Montesinos B., Oudmaijer R. D., Najita J. R., Brittain S. D., van den Ancker M. E., 2014, ApJ, 790, 21

Metchev S. A., Hillenbrand L. A., Meyer M. R., 2004, ApJ, 600, 435

Monnier J. D. et al., 2006, ApJ, 647, 444

Muro-Arena G. A. et al., 2020, A\&A, 636, L4

Muto T. et al., 2012, ApJ, 748, L22

Ohashi S. et al., 2018, ApJ, 864, 81

Ohta Y. et al., 2016, PASJ, 68, 53

Okuzumi S., Momose M., Sirono S.-I., Kobayashi H., Tanaka H., 2016, ApJ, 821,82

Paardekooper S. J., Mellema G., 2006, A\&A, 453, 1129

Pascucci I. et al., 2016, ApJ, 831, 125

Perez S. et al., 2015, ApJ, 798, 85

Pérez L. M. et al., 2016, Science, 353, 1519

Pérez L. M., Isella A., Carpenter J. M., Chandler C. J., 2014, ApJ, 783, L13

Pineda J. E. et al., 2019, ApJ, 871, 48

Pinilla P. et al., 2018, ApJ, 859, 32

Pinilla P., Benisty M., Birnstiel T., 2012, A\&A, 545, A81

Pinilla P., Flock M., Ovelar M. D. J., Birnstiel T., 2016, A\&A, 596, A81

Pohl A., Pinilla P., Benisty M., Ataiee S., Juhász A., Dullemond C. P., Van Boekel R., Henning T., 2015, MNRAS, 453, 1768

Preibisch T., Mamajek E., 2008, in Reipurth B., ed., Handbook of Star Forming Regions, Volume II, 5, p. 235

Ragusa E., Dipierro G., Lodato G., Laibe G., Price D. J., 2017, MNRAS, 464, 1449

Rodriguez D. R., Kastner J. H., Wilner D., Qi C., 2010, ApJ, 720, 1684

Salpeter E. E., 1955, ApJ, 121, 161

Schneider P. C., France K., Günther H. M., Herczeg G., Robrade J., Bouvier J., McJunkin M., Schmitt J. H. M. M., 2015, A\&A, 584, A51

Segura-Cox D. M. et al., 2020, Nature, 586, 228

Sheehan P. D., Eisner J. A., 2018, ApJ, 857, 18

Siess L., Dufour E., Forestini M., 2000, A\&A, 358, 593

Simon T., Ayres T. R., Redfield S., Linsky J. L., 2002, ApJ, 579, 800

Smith K. W., Balega Y. Y., Duschl W. J., Hofmann K. H., Lachaume R., Preibisch T., Schertl D., Weigelt G., 2005, A\&A, 431, 307

Soderblom D. R., Hillenbrand L. A., Jeffries R. D., Mamajek E. E., Naylor T., 2014, in Beuther H., Klessen R. S., Dullemond C. P., Henning T., eds, Protostars and Planets VI. University of Arizona Press, Tucson, p. 219

Stapelfeldt K. R. et al., 1999, ApJ, 516, L95

Takahashi S. Z., Inutsuka S.-I., 2016, AJ, 152, 184

Tang Y. W., Guilloteau S., Piétu V., Dutrey A., Ohashi N., Ho P. T. P., 2012, A\&A, 547, A84

Tang Y.-W. et al., 2017, ApJ, 840, 32

Tanii R. et al., 2012, PASJ, 64, 124 
Tripathi A., Andrews S. M., Birnstiel T., Wilner D. J., 2017, ApJ, 845, 44 Ubeira Gabellini M. G. et al., 2019, MNRAS, 486, 4638

van den Ancker M. E., The P. S., Tjin A Djie H. R. E., Catala C., de Winter D., Blondel P. F. C., Waters L. B. F. M., 1997, A\&A, 324, L33

van der Marel N. et al., 2013, Science, 340, 1199

van der Marel N. et al., 2021, AJ, 161, 33

van der Marel N., van Dishoeck E. F., Bruderer S., Pérez L., Isella A., 2015, A\&A, 579, A106

van der Marel N., van Dishoeck E. F., Bruderer S., Pinilla P., van Kempen T., Perez L., Isella A., 2016a, in Kastner J. H., Stelzer B., Metchev S. A., eds, Young Stars \& Planets Near the Sun, vol. 314, p. 139

van der Marel N., van Dishoeck E. F., Bruderer S., Andrews S. M., Pontoppidan K. M., Herczeg G. J., van Kempen T., Miotello A., 2016b, A\&A, 585, A58

van der Marel N., Williams J. P., Bruderer S., 2018, ApJ, 867, L14

van der Marel N., Dong R., di Francesco J., Williams J. P., Tobin J., 2019, ApJ, 872, 112

van Leeuwen F., 2007, A\&A, 474, 653

van Terwisga S. E. et al., 2018, A\&A, 616, A88

Villenave M. et al., 2019, A\&A, 624, A7

Wagner K. et al., 2018, ApJ, 854, 130

Wagner K., Apai D., Kasper M., Robberto M., 2015, ApJ, 813, L2

Welch W. J., Hartmann L., Helfer T., Briceño C., 2000, ApJ, 540, 362

White R. J., Ghez A. M., Reid I. N., Schultz G., 1999, ApJ, 520, 811
Wilking B. A., Gagné M., Allen L. E., 2008, in Reipurth B., ed., Handbook of Star Forming Regions, Volume II, p. 351

Zhang K., Blake G. A., Bergin E. A., 2015, ApJ, 806, L7

Zhu Z., Dong R., Stone J. M., Rafikov R. R., 2015, ApJ, 813, 88

Zinnecker H., Yorke H. W., 2007, ARA\&A, 45, 481

\section{SUPPORTING INFORMATION}

Supplementary data are available at MNRAS online.

\section{suppl_data}

Please note: Oxford University Press is not responsible for the content or functionality of any supporting materials supplied by the authors. Any queries (other than missing material) should be directed to the corresponding author for the article.

\section{APPENDIX A: COMPLETE LIST OF STUDIED PROTOPLANETARY DISCS}

Table A1 shows the complete list of images we have looked at in our search for protoplanetary discs featuring substructure. Only continuum observations were looked at as we are interested in substructure present in the dust populations of the discs. The complete version of Table A1 can be found online. 
Table A1. The complete sample of the ALMA images we have looked at in our search for protoplanetary rings with substructure. We have listed major star-forming regions only. All distances have been obtained from Gaia DR2 (Gaia Collaboration 2018), unless otherwise stated.

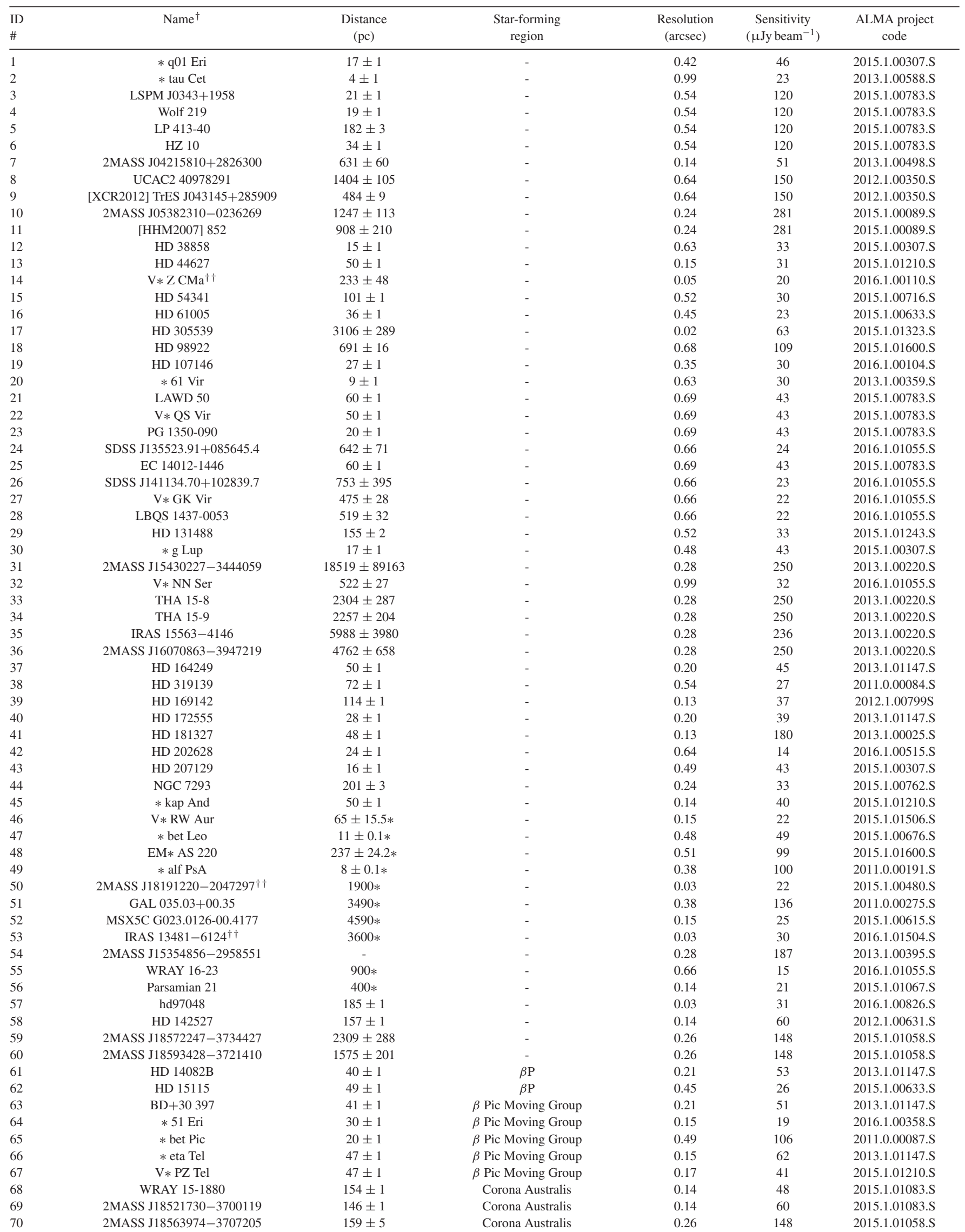


Table A1 - continued

\begin{tabular}{|c|c|c|c|c|c|c|}
\hline $\begin{array}{l}\text { ID } \\
\#\end{array}$ & Name $^{\dagger}$ & $\begin{array}{c}\text { Distance } \\
(\mathrm{pc})\end{array}$ & $\begin{array}{l}\text { Star-forming } \\
\text { region }\end{array}$ & $\begin{array}{l}\text { Resolution } \\
(\operatorname{arcsec})\end{array}$ & $\begin{array}{c}\text { Sensitivity } \\
\left(\mu \mathrm{Jy} \text { beam }^{-1}\right)\end{array}$ & $\begin{array}{l}\text { ALMA project } \\
\text { code }\end{array}$ \\
\hline 71 & 2MASS J18564024-3655203 & $149 \pm 3$ & Corona Australis & 0.26 & 148 & 2015.1.01058.S \\
\hline 72 & 2MASSW J1858509-370631 & $155 \pm 8$ & Corona Australis & 0.26 & 148 & 2015.1.01058.S \\
\hline 73 & 2MASS J19002906-3656036 & $155 \pm 9$ & Corona Australis & 0.64 & 152 & 2011.0.00733.S \\
\hline 74 & 2MASS J19005804-3645048 & $153 \pm 1$ & Corona Australis & 0.60 & 181 & 2011.0.00733.S \\
\hline 75 & 2MASS J19005974-3647109 & $144 \pm 6$ & Corona Australis & 0.26 & 148 & 2015.1.01058.S \\
\hline 76 & 2MASS J19011149-3645337 & $154 \pm 5$ & Corona Australis & 0.26 & 148 & 2015.1.01058.S \\
\hline 77 & $\mathrm{~V} * \mathrm{~V} 667 \mathrm{CrA}$ & $156 \pm 3$ & Corona Australis & 0.26 & 148 & 2015.1.01058.S \\
\hline 78 & 2MASS J19011893-3658282 & $149 \pm 5$ & Corona Australis & 0.26 & 148 & 2015.1.01058.S \\
\hline 79 & 2MASS J19012901-3701484 & $153 \pm 2$ & Corona Australis & 0.64 & 152 & 2011.0.00733.S \\
\hline 80 & HD 176386 & $159 \pm 2$ & Corona Australis & 0.26 & 148 & 2015.1.01058.S \\
\hline 81 & $\mathrm{~V} * \mathrm{TY} \mathrm{CrA}$ & $136 \pm 3$ & Corona Australis & 0.26 & 148 & 2015.1.01058.S \\
\hline 82 & 2MASS J19015374-3700339 & $147 \pm 6$ & Corona Australis & 0.26 & 148 & 2015.1.01058.S \\
\hline 83 & $\mathrm{~V} * \mathrm{DG} \mathrm{CrA}$ & $157 \pm 5$ & Corona Australis & 0.26 & 148 & 2015.1.01058.S \\
\hline 84 & 2MASS J19021201-3703093 & $159 \pm 4$ & Corona Australis & 0.26 & 148 & 2015.1.01058.S \\
\hline 85 & 2MASS J19021667-3645493 & $155 \pm 3$ & Corona Australis & 0.26 & 148 & 2015.1.01058.S \\
\hline 86 & SSTgbs J1902330-365821 & $139 \pm 4$ & Corona Australis & 0.26 & 148 & 2015.1.01058.S \\
\hline 87 & ISO-CrA 177 & $282 \pm 39$ & Corona Australis & 0.26 & 148 & 2015.1.01058.S \\
\hline 88 & VSST 10 & $164 \pm 8$ & Corona Australis & 0.26 & 148 & 2015.1.01058.S \\
\hline 89 & 2MASS J19032429-3715076 & $148 \pm 35$ & Corona Australis & 0.26 & 148 & 2015.1.01058.S \\
\hline 90 & 2MASS J19032547-3655051 & $154 \pm 4$ & Corona Australis & 0.26 & 148 & 2015.1.01058.S \\
\hline 91 & $\mathrm{~V} * \mathrm{~V} 721 \mathrm{CrA}$ & $155 \pm 2$ & Corona Australis & 0.24 & 24 & 2015.1.01301.S \\
\hline 92 & 2MASS J18570785-3654041 & $148 * *$ & Corona Australis & 0.26 & 148 & 2015.1.01058.S \\
\hline 93 & [QZM2013] MM3 & $148 * *$ & Corona Australis & 0.38 & 136 & $2011.0 .00275 . S$ \\
\hline 94 & [WGL92] iras 10 & $148 * *$ & Corona Australis & 0.26 & 148 & 2015.1.01058.S \\
\hline 95 & [LEM2005b] CrA 444b & $148 * *$ & Corona Australis & 0.26 & 148 & 2015.1.01058.S \\
\hline 96 & $\mathrm{~V} * \mathrm{~S} \mathrm{CrA}$ & $148 * *$ & Corona Australis & 0.26 & 148 & 2015.1.01058.S \\
\hline 97 & HH 730A & $148 * *$ & Corona Australis & 0.26 & 148 & 2015.1.01058.S \\
\hline 98 & 2MASS J19013232-3658030 & $148 * *$ & Corona Australis & 0.26 & 148 & 2015.1.01058.S \\
\hline 99 & 2MASS J19013385-3657448 & $148 * *$ & Corona Australis & 0.26 & 148 & 2015.1.01058.S \\
\hline 100 & MHO 2008 & $148 * *$ & Corona Australis & 0.26 & 148 & 2015.1.01058.S \\
\hline 101 & 2MASS J19014041-3651422 & $148 * *$ & Corona Australis & 0.26 & 148 & 2015.1.01058.S \\
\hline 102 & 2MASS J19015180-3710478 & $148 * *$ & Corona Australis & 0.26 & 148 & 2015.1.01058.S \\
\hline 103 & AX $1858.4-3700$ & $148 * *$ & Corona Australis & 0.26 & 148 & 2015.1.01058.S \\
\hline 104 & 2MASS J19015173-3655143 & $148 * *$ & Corona Australis & 0.26 & 148 & 2015.1.01058.S \\
\hline 105 & 2MASS J19015112-3654122 & $148 * *$ & Corona Australis & 0.26 & 148 & 2015.1.01058.S \\
\hline 106 & [SHK2011b] 9 & $148 * *$ & Corona Australis & 0.26 & 148 & 2015.1.01058.S \\
\hline 107 & $\mathrm{~V} * \mathrm{~T} \mathrm{CrA}$ & $148 * *$ & Corona Australis & 0.26 & 148 & 2015.1.01058.S \\
\hline 108 & 2MASS J19020410-3657013 & $148 * *$ & Corona Australis & 0.26 & 148 & 2015.1.01058.S \\
\hline 109 & 2MASS J19020682-3658411 & $148 * *$ & Corona Australis & 0.26 & 148 & 2015.1.01058.S \\
\hline 110 & 2MASS J19021464-3700328 & $148 * *$ & Corona Australis & 0.26 & 148 & 2015.1.01058.S \\
\hline 111 & 2MASS J19022708-3658132 & $148 * *$ & Corona Australis & 0.26 & 148 & 2015.1.01058.S \\
\hline 112 & 2MASS J19031185-3709020 & $148 * *$ & Corona Australis & 0.60 & 181 & 2011.0.00733.S \\
\hline 113 & 2MASS J19041725-3659030 & $148 * *$ & Corona Australis & 0.26 & 148 & 2015.1.01058.S \\
\hline 114 & $\mathrm{~V} * \mathrm{DX}$ Cha & $108 \pm 1$ & Chamaeleon & 0.20 & 70 & 2013.1.00592.S \\
\hline 115 & IRAS $12535-7623$ & $160 \pm 11$ & Chamaeleon & 0.21 & 123 & 2013.1.00708.S \\
\hline 116 & SZ 49 & $195 \pm 2$ & Chamaeleon & 0.21 & 123 & 2013.1.00708.S \\
\hline 117 & SZ 50 & $147 \pm 13$ & Chamaeleon & 0.21 & 123 & 2013.1.00708.S \\
\hline 118 & $\mathrm{~V} * \mathrm{SX}$ Cha & $196 * *$ & Chamaeleon & 0.35 & 911 & 2013.1.00437.S \\
\hline 119 & SZ 4 & $196 * *$ & Chamaeleon & 0.35 & 911 & 2013.1.00437.S \\
\hline 120 & CHXR 9C & $196 * *$ & Chamaeleon & 0.37 & 215 & $2013.1 .01075 . S$ \\
\hline 121 & HH 48 & $196 * *$ & Chamaeleon & 0.28 & 180 & 2016.1.00460.S \\
\hline 122 & Hn 4 & $196 * *$ & Chamaeleon & 0.37 & 215 & 2013.1.01075.S \\
\hline 123 & CHXR 15 & $196 * *$ & Chamaeleon & 0.37 & 215 & 2013.1.01075.S \\
\hline 124 & 2MASS J11062942-7724586 & $196 * *$ & Chamaeleon & 0.35 & 320 & 2013.1.00437.S \\
\hline 125 & [CCE98] 2-21 & $196 * *$ & Chamaeleon & 0.35 & 320 & 2013.1.00437.S \\
\hline 126 & HH 927 & $196 * *$ & Chamaeleon & 0.37 & 215 & 2013.1.01075.S \\
\hline 127 & V* VV Cha & $196 * *$ & Chamaeleon & 0.35 & 911 & 2013.1.00437.S \\
\hline 128 & V* HK Cha & $196 * *$ & Chamaeleon & 0.35 & 320 & 2013.1.00437.S \\
\hline 129 & Glass H & $196 * *$ & Chamaeleon & 0.37 & 215 & 2013.1.01075.S \\
\hline 130 & V* VW Cha & $196 * *$ & Chamaeleon & 0.35 & 320 & 2013.1.00437.S \\
\hline 131 & ESO-HA 562 & $196 * *$ & Chamaeleon & 0.35 & 320 & 2013.1.00437.S \\
\hline 132 & $\mathrm{~V} * \mathrm{HPCha}{ }^{\dagger \dagger}$ & $196 * *$ & Chamaeleon & 0.02 & 16 & $2017.1 .01460 . S$ \\
\hline 133 & 2MASS J11082570-7716396 & $196 * * *$ & Chamaeleon & 0.35 & 320 & 2013.1.00437.S \\
\hline 134 & V* HU Cha & $196 * *$ & Chamaeleon & 0.35 & 320 & 2013.1.00437.S \\
\hline 135 & $\mathrm{~V} * \mathrm{HX}$ Cha & $196 * *$ & Chamaeleon & 0.35 & 320 & 2013.1.00437.S \\
\hline 136 & V* WX Cha & $196 * *$ & Chamaeleon & 0.35 & 320 & 2013.1.00437.S \\
\hline 137 & OTS 44 & $196 * *$ & Chamaeleon & 0.61 & 17 & 2015.1.00243.S \\
\hline 138 & Glass Q & $196 * *$ & Chamaeleon & 0.35 & 320 & 2013.1.00437.S \\
\hline 139 & ESO-HA 569 & $196 * *$ & Chamaeleon & 0.35 & 320 & 2013.1.00437.S \\
\hline 140 & CHX 18 & $196 * *$ & Chamaeleon & 0.37 & 215 & 2013.1.01075.S \\
\hline 141 & 2MASS J11160287-7624533 & $196 * *$ & Chamaeleon & 0.35 & 911 & 2013.1.00437.S \\
\hline
\end{tabular}


Table A1 - continued

\begin{tabular}{|c|c|c|c|c|c|c|}
\hline $\begin{array}{l}\text { ID } \\
\#\end{array}$ & $\mathrm{Name}^{\dagger}$ & $\begin{array}{l}\text { Distance } \\
(\mathrm{pc})\end{array}$ & $\begin{array}{l}\text { Star-forming } \\
\text { region }\end{array}$ & $\begin{array}{l}\text { Resolution } \\
\quad(\operatorname{arcsec})\end{array}$ & $\begin{array}{c}\text { Sensitivity } \\
\left(\mu \mathrm{Jy} \text { beam }^{-1}\right)\end{array}$ & $\begin{array}{l}\text { ALMA project } \\
\text { code }\end{array}$ \\
\hline 142 & 2MASS J11175211-7629392 & $196 * *$ & Chamaeleon & 0.37 & 320 & 2013.1.00437.S \\
\hline 143 & CHXR 68 & $196 * *$ & Chamaeleon & 0.37 & 215 & 2013.1.01075.S \\
\hline 144 & IRAS F11171-7919 & $209 * *$ & Chamaeleon & 0.37 & 320 & 2013.1.00437.S \\
\hline 145 & 2MASS J12534285-7715114 & $209 * *$ & Chamaeleon & 0.21 & 123 & 2013.1.00708.S \\
\hline 146 & 2MASS J12580676-7709094 & $209 * *$ & Chamaeleon & 0.21 & 123 & 2013.1.00708.S \\
\hline 147 & SSTc2d J130529.0-774140 & $209 * *$ & Chamaeleon & 0.21 & 123 & 2013.1.00708.S \\
\hline 148 & $\mathrm{~V} * \mathrm{BK}$ Cha & $209 * *$ & Chamaeleon & 0.21 & 123 & 2013.1.00708.S \\
\hline 149 & SZ 62 & $209 * *$ & Chamaeleon & 0.21 & 123 & 2013.1.00708.S \\
\hline 150 & 2MASS J10533978-7712338 & $192 \pm 4$ & Chamaeleon & 0.35 & 911 & 2013.1.00437.S \\
\hline 151 & 2MASS J10561638-7630530 & $196 \pm 4$ & Chamaeleon & 0.37 & 320 & 2013.1.00437.S \\
\hline 152 & $\mathrm{~V} * \mathrm{SY}$ Cha & $183 \pm 1$ & Chamaeleon & 0.35 & 911 & 2013.1.00437.S \\
\hline 153 & 2MASS J10580597-7711501 & $187 \pm 4$ & Chamaeleon & 0.35 & 320 & 2013.1.00437.S \\
\hline 154 & $\mathrm{~V} * \mathrm{SZ}$ Cha & $190 \pm 1$ & Chamaeleon & 0.35 & 320 & 2013.1.00437.S \\
\hline 155 & V* TW Cha & $185 \pm 1$ & Chamaeleon & 0.35 & 911 & 2013.1.00437.S \\
\hline 156 & Hen 3-545 & $187 \pm 1$ & Chamaeleon & 0.35 & 911 & 2013.1.00437.S \\
\hline 157 & Ass Cha T 1-6 & $192 \pm 2$ & Chamaeleon & 0.37 & 320 & 2013.1.00437.S \\
\hline 158 & V* CS Cha & $176 \pm 1$ & Chamaeleon & 0.03 & 33 & 2017.1.00969.S \\
\hline 159 & Hn 1 & $195 \pm 6$ & Chamaeleon & 0.35 & 320 & 2013.1.00437.S \\
\hline 160 & Ass Cha T $1-8$ & $182 \pm 2$ & Chamaeleon & 0.35 & 320 & 2013.1.00437.S \\
\hline 161 & CHSM 1715 & $192 \pm 3$ & Chamaeleon & 0.37 & 320 & 2013.1.00437.S \\
\hline 162 & $\mathrm{~V} * \mathrm{CT}$ Cha & $192 \pm 1$ & Chamaeleon & 0.35 & 911 & 2013.1.00437.S \\
\hline 163 & BYB 18 & $193 \pm 3$ & Chamaeleon & 0.37 & 320 & 2013.1.00437.S \\
\hline 164 & SZ 13 & $194 \pm 3$ & Chamaeleon & 0.35 & 911 & 2013.1.00437.S \\
\hline 165 & 2MASS J11062554-7633418 & $209 \pm 5$ & Chamaeleon & 0.37 & 320 & 2013.1.00437.S \\
\hline 166 & CHXR 73 & $191 \pm 6$ & Chamaeleon & 0.37 & 215 & 2013.1.01075.S \\
\hline 167 & CHSM 7869 & $187 \pm 7$ & Chamaeleon & 0.37 & 320 & 2013.1.00437.S \\
\hline 168 & ISO-ChaI 79 & $205 \pm 22$ & Chamaeleon & 0.35 & 320 & 2013.1.00437.S \\
\hline 169 & Hn 5 & $195 \pm 2$ & Chamaeleon & 0.35 & 320 & 2013.1.00437.S \\
\hline 170 & $\mathrm{~V} * \mathrm{UX}$ Cha & $185 \pm 1$ & Chamaeleon & 0.35 & 320 & 2013.1.00437.S \\
\hline 171 & $\mathrm{~V} * \mathrm{UY}$ Cha & $190 \pm 2$ & Chamaeleon & 0.35 & 320 & 2013.1.00437.S \\
\hline 172 & 2MASS J11065939-7530559 & $196 \pm 4$ & Chamaeleon & 0.37 & 320 & 2013.1.00437.S \\
\hline 173 & $\mathrm{~V} * \mathrm{UZ}$ Cha & $196 \pm 1$ & Chamaeleon & 0.35 & 320 & 2013.1.00437.S \\
\hline 174 & CHSM 9484 & $199 \pm 4$ & Chamaeleon & 0.35 & 320 & 2013.1.00437.S \\
\hline 175 & [CCE98] 1-46 & $173 \pm 7$ & Chamaeleon & 0.35 & 911 & 2013.1.00437.S \\
\hline 176 & [NC98] Cha HA 9 & $199 \pm 12$ & Chamaeleon & 0.35 & 320 & 2013.1.00437.S \\
\hline 177 & V* DI Cha & $191 \pm 1$ & Chamaeleon & 0.35 & 320 & 2013.1.00437.S \\
\hline 178 & CHXR 76 & $189 \pm 3$ & Chamaeleon & 0.42 & 122 & 2012.1.00350.S \\
\hline 179 & Ass Cha T 1-15 & $195 \pm 2$ & Chamaeleon & 0.35 & 320 & 2013.1.00437.S \\
\hline 180 & 2MASS J11074656-7615174 & $194 \pm 7$ & Chamaeleon & 0.37 & 320 & 2013.1.00437.S \\
\hline 181 & SZ 23 & $184 \pm 2$ & Chamaeleon & 0.35 & 320 & 2013.1.00437.S \\
\hline 182 & Ass Cha T 1-16 & $163 \pm 8$ & Chamaeleon & 0.35 & 320 & 2013.1.00437.S \\
\hline 183 & V* HM Cha & $187 \pm 12$ & Chamaeleon & 0.35 & 320 & 2013.1.00437.S \\
\hline 184 & CHXR 30A & $253 \pm 26$ & Chamaeleon & 0.35 & 320 & 2013.1.00437.S \\
\hline 185 & ISO-ChaI 138 & $186 \pm 7$ & Chamaeleon & 0.35 & 320 & 2013.1.00437.S \\
\hline 186 & ISO-ChaI 143 & $193 \pm 5$ & Chamaeleon & 0.35 & 320 & 2013.1.00437.S \\
\hline 187 & ISO-ChaI 147 & $200 \pm 8$ & Chamaeleon & 0.35 & 320 & 2013.1.00437.S \\
\hline 188 & V* HQ Cha & $179 \pm 4$ & Chamaeleon & 0.35 & 320 & 2013.1.00437.S \\
\hline 189 & Ass Cha T $1-20$ & $188 \pm 2$ & Chamaeleon & 0.35 & 320 & 2013.1.00437.S \\
\hline 190 & SZ 28 & $193 \pm 3$ & Chamaeleon & 0.35 & 320 & 2013.1.00437.S \\
\hline 191 & $\mathrm{~V} * \mathrm{PU}$ Car & $188 \pm 1$ & Chamaeleon & 0.35 & 911 & 2013.1.00437.S \\
\hline 192 & V*VY Cha & $186 \pm 2$ & Chamaeleon & 0.35 & 911 & 2013.1.00437.S \\
\hline 193 & $\mathrm{~V} *$ HS Cha & $195 \pm 4$ & Chamaeleon & 0.35 & 320 & 2013.1.00437.S \\
\hline 194 & NAME Sz 30A & $197 \pm 5$ & Chamaeleon & 0.37 & 215 & 2013.1.01075.S \\
\hline 195 & V* HV Cha & $203 \pm 8$ & Chamaeleon & 0.35 & 320 & 2013.1.00437.S \\
\hline 196 & $\mathrm{~V} * \mathrm{VZ}$ Cha & $192 \pm 1$ & Chamaeleon & 0.35 & 320 & 2013.1.00437.S \\
\hline 197 & HJM C 7-1 & $188 \pm 6$ & Chamaeleon & 0.35 & 320 & 2013.1.00437.S \\
\hline 198 & HJM C 1-24 & $195 \pm 3$ & Chamaeleon & 0.35 & 320 & 2013.1.00437.S \\
\hline 199 & BYB 43 & $193 \pm 5$ & Chamaeleon & 0.35 & 320 & 2013.1.00437.S \\
\hline 200 & HD 97300 & $193 \pm 1$ & Chamaeleon & 0.37 & 215 & 2013.1.01075.S \\
\hline 201 & ISO-ChaI 220 & $186 \pm 16$ & Chamaeleon & 0.35 & 320 & 2013.1.00437.S \\
\hline 202 & ISO-ChaI 217 & $240 \pm 13$ & Chamaeleon & 0.35 & 320 & 2013.1.00437.S \\
\hline 203 & Ass Cha T 1-23 & $202 \pm 6$ & Chamaeleon & 0.35 & 320 & 2013.1.00437.S \\
\hline 204 & V* WW Cha & $192 \pm 1$ & Chamaeleon & 0.35 & 320 & 2013.1.00437.S \\
\hline 205 & $\mathrm{~V} * \mathrm{FN}$ Cha & $195 \pm 1$ & Chamaeleon & 0.35 & 320 & 2013.1.00437.S \\
\hline 206 & HJM C 1-4 & $201 \pm 3$ & Chamaeleon & 0.35 & 320 & 2013.1.00437.S \\
\hline 207 & 2MASS J11100785-7727480 & $200 \pm 13$ & Chamaeleon & 0.35 & 320 & 2013.1.00437.S \\
\hline 208 & V* WY Cha & $180 \pm 9$ & Chamaeleon & 0.35 & 911 & 2013.1.00437.S \\
\hline 209 & HJM C 1-8 & $195 \pm 2$ & Chamaeleon & 0.35 & 320 & 2013.1.00437.S \\
\hline 210 & ISO-ChaI 252 & $204 \pm 12$ & Chamaeleon & 0.35 & 320 & 2013.1.00437.S \\
\hline 211 & Ass Cha T $1-27$ & $185 \pm 1$ & Chamaeleon & 0.35 & 320 & 2013.1.00437.S \\
\hline 212 & V* IK Cha & $196 \pm 11$ & Chamaeleon & 0.35 & 320 & 2013.1.00437.S \\
\hline
\end{tabular}


Table A1 - continued

\begin{tabular}{|c|c|c|c|c|c|c|}
\hline $\begin{array}{l}\text { ID } \\
\#\end{array}$ & Name $^{\dagger}$ & $\begin{array}{l}\text { Distance } \\
\quad(\mathrm{pc})\end{array}$ & $\begin{array}{l}\text { Star-forming } \\
\text { region }\end{array}$ & $\begin{array}{l}\text { Resolution } \\
(\operatorname{arcsec})\end{array}$ & $\begin{array}{c}\text { Sensitivity } \\
\left(\mu \mathrm{Jy} \text { beam }^{-1}\right)\end{array}$ & $\begin{array}{l}\text { ALMA project } \\
\text { code }\end{array}$ \\
\hline 213 & V* WZ Cha & $195 \pm 1$ & Chamaeleon & 0.35 & 911 & 2013.1.00437.S \\
\hline 214 & HJM C 2-5 & $140 \pm 14$ & Chamaeleon & 0.35 & 320 & 2013.1.00437.S \\
\hline 215 & V* XX Cha & $191 \pm 1$ & Chamaeleon & 0.35 & 911 & 2013.1.00437.S \\
\hline 216 & CHX 18N & $193 \pm 1$ & Chamaeleon & 0.35 & 911 & 2013.1.00437.S \\
\hline 217 & V* IM Cha & $185 \pm 4$ & Chamaeleon & 0.37 & 320 & 2013.1.00437.S \\
\hline 218 & SZ 40 & $193 \pm 2$ & Chamaeleon & 0.35 & 320 & 2013.1.00437.S \\
\hline 219 & SZ 41 & $194 \pm 1$ & Chamaeleon & 0.35 & 320 & 2013.1.00437.S \\
\hline 220 & V* CV Cha & $193 \pm 1$ & Chamaeleon & 0.35 & 320 & 2013.1.00437.S \\
\hline 221 & V* CW Cha & $196 \pm 2$ & Chamaeleon & 0.35 & 320 & 2013.1.00437.S \\
\hline 222 & Ass Cha T 2-54 & $202 \pm 17$ & Chamaeleon & 0.42 & 122 & 2012.1.00350.S \\
\hline 223 & HJM E 2-9 & $191 \pm 2$ & Chamaeleon & 0.35 & 320 & 2013.1.00437.S \\
\hline 224 & Hn 18 & $190 \pm 2$ & Chamaeleon & 0.35 & 911 & 2013.1.00437.S \\
\hline 225 & Hn $21 \mathrm{~W}$ & $189 \pm 3$ & Chamaeleon & 0.37 & 320 & 2013.1.00437.S \\
\hline 226 & Hn 21 & $189 \pm 3$ & Chamaeleon & 0.37 & 215 & 2013.1.01075.S \\
\hline 227 & BYB 53 & $194 \pm 7$ & Chamaeleon & 0.37 & 215 & 2013.1.01075.S \\
\hline 228 & Ass Cha T 1-32 & $188 \pm 1$ & Chamaeleon & 0.35 & 911 & 2013.1.00437.S \\
\hline 229 & 2MASS J11241186-7630425 & $185 \pm 2$ & Chamaeleon & 0.37 & 320 & 2013.1.00437.S \\
\hline 230 & [FLG2003] eps Cha 17 & $181 \pm 18$ & Chamaeleon & 0.37 & 320 & 2013.1.00437.S \\
\hline 231 & $\mathrm{~V} * \mathrm{~T}$ Cha & $110 \pm 1$ & Chamaeleon & 0.13 & 90 & 2012.2.00182.S \\
\hline 232 & V* DK Cha & $244 \pm 22$ & Chamaeleon & 0.21 & 123 & 2013.1.00708.S \\
\hline 233 & NAME Sz 46N & $196 \pm 2$ & Chamaeleon & 0.21 & 123 & 2013.1.00708.S \\
\hline 234 & 2MASS J13005927-7714027 & $220 \pm 10$ & Chamaeleon & 0.21 & 123 & 2013.1.00708.S \\
\hline 235 & SZ 51 & $199 \pm 1$ & Chamaeleon & 0.21 & 123 & 2013.1.00708.S \\
\hline 236 & $\mathrm{~V} * \mathrm{CM}$ Cha & $194 \pm 1$ & Chamaeleon & 0.21 & 123 & 2013.1.00708.S \\
\hline 237 & 2MASS J13022287-7734494 & $205 \pm 4$ & Chamaeleon & 0.21 & 123 & 2013.1.00708.S \\
\hline 238 & SZ 52 & $204 \pm 3$ & Chamaeleon & 0.21 & 123 & 2013.1.00708.S \\
\hline 239 & Hn 22 & $199 \pm 1$ & Chamaeleon & 0.21 & 123 & 2013.1.00708.S \\
\hline 240 & Hn 24 & $197 \pm 1$ & Chamaeleon & 0.21 & 123 & 2013.1.00708.S \\
\hline 241 & Hn 25 & $197 \pm 3$ & Chamaeleon & 0.21 & 123 & 2013.1.00708.S \\
\hline 242 & SZ 53 & $197 \pm 2$ & Chamaeleon & 0.21 & 123 & 2013.1.00708.S \\
\hline 243 & Hen 3-854 & $197 \pm 1$ & Chamaeleon & 0.21 & 123 & 2013.1.00708.S \\
\hline 244 & 2MASS J13052169-7738102 & $202 \pm 5$ & Chamaeleon & 0.21 & 123 & 2013.1.00708.S \\
\hline 245 & SZ 56 & $191 \pm 2$ & Chamaeleon & 0.21 & 123 & 2013.1.00708.S \\
\hline 246 & SZ 58 & $186 \pm 2$ & Chamaeleon & 0.21 & 123 & 2013.1.00708.S \\
\hline 247 & 2MASS J13071806-7740529 & $199 \pm 5$ & Chamaeleon & 0.21 & 123 & 2013.1.00708.S \\
\hline 248 & Hn 26 & $198 \pm 3$ & Chamaeleon & 0.21 & 123 & 2013.1.00708.S \\
\hline 249 & $\mathrm{~V} * \mathrm{BM}$ Cha & $202 \pm 2$ & Chamaeleon & 0.21 & 123 & 2013.1.00708.S \\
\hline 250 & 2MASS J13082714-7743232 & $205 \pm 6$ & Chamaeleon & 0.21 & 123 & 2013.1.00708.S \\
\hline 251 & SZ 63 & $202 \pm 1$ & Chamaeleon & 0.21 & 123 & 2013.1.00708.S \\
\hline 252 & $\mathrm{Cl} *$ Trumpler $14 \mathrm{VBF} 125$ & $3000 * *$ & Carina Nebula & 0.02 & 63 & 2015.1.01323.S \\
\hline 253 & [SBM2003] J104405.4-592940 & $3000 * *$ & Carina Nebula & 0.02 & 63 & 2015.1.01323.S \\
\hline 254 & [GG2014] 230 & $3000 * *$ & Carina Nebula & 0.02 & 63 & 2015.1.01323.S \\
\hline 255 & HD 21997 & $70 \pm 1$ & Columba Association & 0.98 & 85 & 2011.0.00780.S \\
\hline 256 & HD 100453 & $104 \pm 1$ & DC296.27.9 & 0.03 & 25 & 2017.1.01424.S \\
\hline 257 & HD 100546 & $110 \pm 1$ & DC296.27.9 & 0.03 & 57 & 2015.1.00806.S \\
\hline 258 & $\mathrm{~V} * \mathrm{EH}$ Cha & $99 \pm 1$ & $\eta$ Chamaeleontis Association & 0.42 & 122 & 2012.1.00350.S \\
\hline 259 & $\mathrm{~V} * \mathrm{EI}$ Cha & $100 \pm 1$ & $\eta$ Chamaeleontis Association & 0.52 & 122 & 2012.1.00350.S \\
\hline 260 & $\mathrm{~V} *$ ET Cha & $92 \pm 3$ & $\eta$ Chamaeleontis Association & 0.40 & 184 & 2011.0.00133.S \\
\hline 261 & S255IR SMA1 & $200000 * *$ & Gemini & 0.4 & 42 & 2015.1.00500.S \\
\hline 262 & $\mathrm{~V} * \mathrm{~V} 471 \mathrm{Tau}$ & $48 \pm 1$ & Hydes & 0.54 & 120 & 2015.1.00783.S \\
\hline 263 & HG 7-85 & $42 \pm 1$ & Hydes & 0.54 & 69 & 2015.1.00783.S \\
\hline 264 & EGGR 29 & $50 \pm 1$ & Hydes & 0.54 & 85 & 2015.1.00783.S \\
\hline 265 & $\mathrm{~V} * \mathrm{GW} \mathrm{Ori}^{\dagger \dagger}$ & $402 \pm 11$ & $\lambda$ Orionis Association & 0.10 & 18 & 2017.1.00286.S \\
\hline 266 & HD 121191 & $132 \pm 1$ & Lower Centaurus Crux & 0.50 & 37 & 2015.1.01243.S \\
\hline 267 & HD 121617 & $117 \pm 1$ & Lower Centaurus Crux & 0.50 & 38 & 2015.1.01243.S \\
\hline 268 & CD-40 8434 & $113 \pm 1$ & Lower Centaurus Crux & 0.05 & 30 & 2017.A.00006.S \\
\hline 269 & SZ 66 & $157 \pm 2$ & Lupus & 0.28 & 236 & 2013.1.00220.S \\
\hline 270 & 2MASS J15445789-3423392 & $153 \pm 3$ & Lupus & 0.28 & 236 & 2013.1.00220.S \\
\hline 271 & $\mathrm{~V} * \mathrm{HW}$ Lup & $155 \pm 2$ & Lupus & 0.28 & 236 & 2013.1.00220.S \\
\hline 272 & $\mathrm{~V} * \mathrm{GW}$ Lup & $156 \pm 1$ & Lupus & 0.02 & 18 & 2016.1.00484.L \\
\hline 273 & V* HM Lup & $156 \pm 1$ & Lupus & 0.28 & 236 & 2013.1.00220.S \\
\hline 274 & CD-35 10525 & $152 \pm 1$ & Lupus & 0.14 & 45 & 2013.1.00374.S \\
\hline 275 & [L2013] J155140.32-214610.6 & $142 \pm 2$ & Lupus & 0.28 & 187 & 2013.1.00395.S \\
\hline 276 & 2MASS J15521088-2125372 & $168 \pm 8$ & Lupus & 0.28 & 187 & 2013.1.00395.S \\
\hline 277 & [L2013] J155301.32-211413.7 & $146 \pm 3$ & Lupus & 0.28 & 187 & 2013.1.00395.S \\
\hline 278 & NAME THA $15-10 \mathrm{~A}$ & $160 \pm 2$ & Lupus & 0.28 & 236 & 2013.1.00220.S \\
\hline 279 & THA $15-12$ & $158 \pm 1$ & Lupus & 0.39 & 59 & 2013.1.00226.S \\
\hline 280 & $\mathrm{~V} * \mathrm{RU}$ Lup & $160 \pm 2$ & Lupus & 0.02 & 16 & 2016.1.00484.L \\
\hline 281 & SZ 84 & $153 \pm 2$ & Lupus & 0.28 & 236 & 2013.1.00220.S \\
\hline 282 & WRAY 15-1400 & $162 \pm 1$ & Lupus & 0.03 & 15 & 2016.1.00484.L \\
\hline 283 & 2MASS J15592523-4235066 & $147 \pm 2$ & Lupus & 0.28 & 236 & 2013.1.00220.S \\
\hline
\end{tabular}


Table A1 - continued

\begin{tabular}{|c|c|c|c|c|c|c|}
\hline $\begin{array}{l}\text { ID } \\
\#\end{array}$ & Name $^{\dagger}$ & $\begin{array}{c}\text { Distance } \\
(\mathrm{pc})\end{array}$ & $\begin{array}{l}\text { Star-forming } \\
\text { region }\end{array}$ & $\begin{array}{l}\text { Resolution } \\
(\operatorname{arcsec})\end{array}$ & $\begin{array}{c}\text { Sensitivity } \\
\left(\mu \mathrm{Jy} \mathrm{beam}^{-1}\right)\end{array}$ & $\begin{array}{l}\text { ALMA project } \\
\text { code }\end{array}$ \\
\hline 284 & $\mathrm{~V} * \mathrm{RY}$ Lup & $159 \pm 2$ & Lupus & 0.14 & 27 & 2017.1.00449.S \\
\hline 285 & 2MASS J16000060-4221567 & $161 \pm 2$ & Lupus & 0.28 & 236 & 2013.1.00220.S \\
\hline 286 & 2MASS J16000236-4222145 & $164 \pm 2$ & Lupus & 0.28 & 236 & 2013.1.00220.S \\
\hline 287 & 2MASS J16002612-4153553 & $164 \pm 3$ & Lupus & 0.60 & 152 & 2011.0.00733.S \\
\hline 288 & SZ 130 & $160 \pm 1$ & Lupus & 0.28 & 236 & 2013.1.00220.S \\
\hline 289 & $\mathrm{~V} *$ MY Lup & $157 \pm 1$ & Lupus & 0.60 & 152 & 2011.0.00733.S \\
\hline 290 & SZ 131 & $160 \pm 1$ & Lupus & 0.28 & 236 & 2013.1.00220.S \\
\hline 291 & $\mathrm{~V} * \mathrm{MZ}$ Lup & $191 \pm 4$ & Lupus & 0.39 & 100 & $2012.1 .00350 . S$ \\
\hline 292 & $\mathrm{~V} *$ NO Lup & $134 \pm 1$ & Lupus & 0.39 & 100 & 2012.1.00350.S \\
\hline 293 & HD 143675 & $139 \pm 1$ & Lupus & 0.54 & 56 & 2015.1.01243.S \\
\hline 294 & SZ 133 & $153 \pm 13$ & Lupus & 0.28 & 250 & 2013.1.00220.S \\
\hline 295 & V* HO Lup A & $158 \pm 1$ & Lupus & 0.28 & 236 & 2013.1.00220.S \\
\hline 296 & 2MASS J16070854-3914075 & $176 \pm 13$ & Lupus & 0.28 & 236 & 2013.1.00220.S \\
\hline 297 & THA $15-21$ & $160 \pm 1$ & Lupus & 0.28 & 236 & 2013.1.00220.S \\
\hline 298 & THA $15-20$ & $159 \pm 2$ & Lupus & 0.13 & 69 & 2013.1.00663.S \\
\hline 299 & 2MASS J16073773-3921388 & $174 \pm 5$ & Lupus & 0.28 & 236 & 2013.1.00220.S \\
\hline 300 & SZ 95 & $158 \pm 2$ & Lupus & 0.60 & 174 & 2011.0.00733.S \\
\hline 301 & 2MASS J16080017-3902595 & $160 \pm 3$ & Lupus & 0.28 & 236 & 2013.1.00220.S \\
\hline 302 & SZ 96 & $157 \pm 1$ & Lupus & 0.60 & 174 & 2011.0.00733.S \\
\hline 303 & 2MASS J16081497-3857145 & $146 \pm 19$ & Lupus & 0.28 & 236 & 2013.1.00220.S \\
\hline 304 & $\mathrm{~V} * \mathrm{~V} 1279$ Sco & $156 \pm 1$ & Lupus & 0.28 & 236 & 2013.1.00220.S \\
\hline 305 & THA $15-24$ & $158 \pm 2$ & Lupus & 0.28 & 236 & 2013.1.00220.S \\
\hline 306 & THA $15-25$ & $159 \pm 2$ & Lupus & 0.28 & 236 & 2013.1.00220.S \\
\hline 307 & THA $15-26$ & $137 \pm 3$ & Lupus & 0.28 & 236 & 2013.1.00220.S \\
\hline 308 & THA $15-30$ & $165 \pm 2$ & Lupus & 0.28 & 236 & 2013.1.00220.S \\
\hline 309 & IRAS $16051-3820$ & $156 \pm 1$ & Lupus & 0.28 & 250 & 2013.1.00220.S \\
\hline 310 & $\mathrm{~V} * \mathrm{~V} 856 \mathrm{Sco}$ & $161 \pm 2$ & Lupus & 0.47 & 124 & 2015.1.01600.S \\
\hline 311 & $\mathrm{~V} * \mathrm{~V} 856 \mathrm{Sco}$ & $161 \pm 2$ & Lupus & 0.28 & 236 & 2013.1.00220.S \\
\hline 312 & SZ 107 & $152 \pm 3$ & Lupus & 0.60 & 152 & 2011.0.00733.S \\
\hline 313 & V*V1192 Sco & $151 \pm 14$ & Lupus & 0.28 & 236 & 2013.1.00220.S \\
\hline 314 & V*V1193 Sco & $160 \pm 1$ & Lupus & 0.28 & 236 & 2013.1.00220.S \\
\hline 315 & 2MASS J16085373-3914367 & $134 \pm 44$ & Lupus & 0.28 & 236 & 2013.1.00220.S \\
\hline 316 & THA $15-33$ & $158 \pm 1$ & Lupus & 0.28 & 236 & 2013.1.00220.S \\
\hline 317 & SZ 112 & $160 \pm 2$ & Lupus & 0.60 & 152 & 2011.0.00733.S \\
\hline 318 & 2MASS J16085529-3848481 & $158 \pm 3$ & Lupus & 0.28 & 236 & 2013.1.00220.S \\
\hline 319 & THA $15-34$ & $163 \pm 2$ & Lupus & 0.28 & 236 & 2013.1.00220.S \\
\hline 320 & 2MASS J16090141-3925119 & $164 \pm 2$ & Lupus & 0.60 & 174 & 2011.0.00733.S \\
\hline 321 & THA $15-35$ & $162 \pm 1$ & Lupus & 0.28 & 236 & 2013.1.00220.S \\
\hline 322 & SZ 115 & $158 \pm 2$ & Lupus & 0.28 & 236 & 2013.1.00220.S \\
\hline 323 & THA $15-37$ & $159 \pm 1$ & Lupus & 0.28 & 236 & 2013.1.00220.S \\
\hline 324 & SZ 118 & $164 \pm 1$ & Lupus & 0.28 & 236 & 2013.1.00220.S \\
\hline 325 & SONYC Lup3-29 & $193 \pm 7$ & Lupus & 0.28 & 236 & 2013.1.00220.S \\
\hline 326 & 2MASS J16101307-3846165 & $145 \pm 5$ & Lupus & 0.28 & 236 & 2013.1.00220.S \\
\hline 327 & 2MASS J16101984-3836065 & $159 \pm 3$ & Lupus & 0.28 & 236 & 2013.1.00220.S \\
\hline 328 & 2MASS J16102955-3922144 & $163 \pm 2$ & Lupus & 0.60 & 181 & 2011.0.00733.S \\
\hline 329 & 2MASS J16104536-3854547 & $5435 \pm 3072$ & Lupus & 0.28 & 236 & 2013.1.00220.S \\
\hline 330 & NAME THA $15-42 B$ & $1497 \pm 323$ & Lupus & 0.28 & 236 & 2013.1.00220.S \\
\hline 331 & 2MASS J16114865-3817580 & $1812 \pm 263$ & Lupus & 0.28 & 236 & 2013.1.00220.S \\
\hline 332 & 2MASS J16115979-3823383 & $165 \pm 3$ & Lupus & 0.28 & 236 & 2013.1.00220.S \\
\hline 333 & 2MASS J16120445-3809589 & $4630 \pm 14168$ & Lupus & 0.28 & 250 & 2013.1.00220.S \\
\hline 334 & 2MASS J16124373-3815031 & $160 \pm 1$ & Lupus & 0.28 & 250 & 2013.1.00220.S \\
\hline 335 & 2MASS J16134410-3736462 & $160 \pm 2$ & Lupus & 0.28 & 250 & 2013.1.00220.S \\
\hline 336 & HD 145880 & $126 \pm 1$ & Lupus & 0.54 & 51 & 2015.1.01243.S \\
\hline 337 & WRAY 15-1443 & $158 \pm 1$ & Lupus & 0.22 & 925 & 2011.0.00724.S \\
\hline 338 & V1094 Sco & $154 \pm 1$ & Lupus & 0.17 & 61 & 2016.1.01239.S \\
\hline 339 & CPD-36 6759 & $136 \pm 1$ & Lupus & 0.35 & 108 & 2012.1.00870.S \\
\hline 340 & NAME Lupus I & $144 * *$ & Lupus & 0.28 & 250 & 2013.1.00220.S \\
\hline 341 & 2MASS J15450634-3417378 & $144 * *$ & Lupus & 0.28 & 236 & 2013.1.00220.S \\
\hline 342 & [MHR2017] L1S2 9 & $144 * *$ & Lupus & 0.28 & 236 & 2013.1.00220.S \\
\hline 343 & [DB2002b] G339.17+16.08 & $144 * *$ & Lupus & 0.28 & 236 & 2013.1.00220.S \\
\hline 344 & DCld $339.0+15.0$ & $144 * *$ & Lupus & 0.28 & 236 & 2013.1.00220.S \\
\hline 345 & RX J1548.1-3515 & $144 * *$ & Lupus & 0.28 & 236 & 2013.1.00220.S \\
\hline 346 & IRAS $15567-4141$ & $144 * *$ & Lupus & 0.28 & 236 & 2013.1.00220.S \\
\hline 347 & 2MASS J16011549-4152351 & $144 * *$ & Lupus & 0.28 & 250 & 2013.1.00220.S \\
\hline 348 & 2MASS J16070384-3911113 & $144 * *$ & Lupus & 0.28 & 236 & 2013.1.00220.S \\
\hline 349 & 2MASS J16075475-3915446 & $144 * *$ & Lupus & 0.28 & 236 & 2013.1.00220.S \\
\hline 350 & {$[\mathrm{G} 2006] 16$} & $144 * *$ & Lupus & 0.28 & 236 & 2013.1.00220.S \\
\hline 351 & 2MASS J16080618-3912225 & $144 * *$ & Lupus & 0.28 & 236 & 2013.1.00220.S \\
\hline 352 & [GMM2009] Lupus III 33 & $197 * *$ & Lupus 3 & 0.28 & 236 & 2013.1.00220.S \\
\hline 353 & THA $15-27$ & $197 * *$ & Lupus 3 & 0.60 & 152 & 2011.0.00733.S \\
\hline 354 & [MJS2008] 52 & $197 * *$ & Lupus 3 & 0.28 & 236 & 2013.1.00220.S \\
\hline
\end{tabular}


Table A1 - continued

\begin{tabular}{|c|c|c|c|c|c|c|}
\hline $\begin{array}{l}\text { ID } \\
\#\end{array}$ & Name $^{\dagger}$ & $\begin{array}{l}\text { Distance } \\
(\mathrm{pc})\end{array}$ & $\begin{array}{c}\text { Star-forming } \\
\text { region }\end{array}$ & $\begin{array}{c}\text { Resolution } \\
(\operatorname{arcsec})\end{array}$ & $\begin{array}{c}\text { Sensitivity } \\
\left(\mu \mathrm{Jy} \mathrm{beam}^{-1}\right)\end{array}$ & $\begin{array}{l}\text { ALMA project } \\
\text { code }\end{array}$ \\
\hline 355 & 2MASS J16083156-3847292 & $197 * *$ & Lupus 3 & 0.60 & 174 & 2011.0.00733.S \\
\hline 356 & [G2006] 72 & $197 * *$ & Lupus 3 & 0.28 & 236 & 2013.1.00220.S \\
\hline 357 & [SC95] ZET 14 & $197 * *$ & Lupus 3 & 0.28 & 236 & 2013.1.00220.S \\
\hline 358 & 2MASS J16085834-3907491 & $197 * *$ & Lupus 3 & 0.28 & 236 & 2013.1.00220.S \\
\hline 359 & 2MASS J16091644-3904438 & $197 * *$ & Lupus 3 & 0.28 & 236 & 2013.1.00220.S \\
\hline 360 & [BPB2012] Lup3 C3 & $197 * *$ & Lupus 3 & 0.28 & 236 & 2013.1.00220.S \\
\hline 361 & 2MASS J16092032-3904015 & $197 * *$ & Lupus 3 & 0.28 & 236 & 2013.1.00220.S \\
\hline 362 & 2MASS J16092317-3904074 & $197 * *$ & Lupus 3 & 0.28 & 236 & 2013.1.00220.S \\
\hline 363 & 2MASS J16092697-3836269 & $197 * *$ & Lupus 3 & 0.28 & 250 & 2013.1.00220.S \\
\hline 364 & [MJS2008] 94 & $197 * *$ & Lupus 3 & 0.28 & 236 & 2013.1.00220.S \\
\hline 365 & [BPB2012] Lup3 C5 & $197 * *$ & Lupus 3 & 0.28 & 236 & 2013.1.00220.S \\
\hline 366 & 2MASS J16095399-3923275 & $197 * *$ & Lupus 3 & 0.60 & 181 & 2011.0.00733.S \\
\hline 367 & 2MASS J16095628-3859518 & $197 * *$ & Lupus 3 & 0.28 & 236 & 2013.1.00220.S \\
\hline 368 & 2MASS J16102741-3902299 & $197 * *$ & Lupus 3 & 0.28 & 236 & 2013.1.00220.S \\
\hline 369 & 2MASS J16121120-3832197 & $197 * *$ & Lupus 3 & 0.28 & 236 & 2013.1.00220.S \\
\hline 370 & 2MASS J16122269-3713276 & $197 * *$ & Lupus 3 & 0.28 & 250 & 2013.1.00220.S \\
\hline 371 & WRAY 16-203 & $422 \pm 96 * * *$ & Lupus & 0.37 & 58 & 2012.1.00857.S \\
\hline 372 & 2MASS J16164198-3650456 & $38462 \pm 178994 * * *$ & Lupus & 0.77 & 35 & 2015.1.00791.S \\
\hline 373 & 2MASS J16232807-4015368 & $19231 \pm 37721 * * *$ & Lupus & 0.77 & 35 & 2015.1.00791.S \\
\hline 374 & $\mathrm{~V} * \mathrm{~V} 1003 \mathrm{Oph}$ & $117 \pm 1$ & Ophiuchus & 0.52 & 305 & 2011.0.00531.S \\
\hline 375 & Hen 3-1258 & $124 \pm 1$ & Ophiuchus & 0.03 & 16 & 2016.1.00484.L \\
\hline 376 & $\mathrm{EM} * \mathrm{AS} 209$ & $121 \pm 1$ & Ophiuchus & 0.15 & 41 & 2015.1.00486.S \\
\hline 377 & Haro 1-16 & $146 \pm 1$ & Ophiuchus & 0.23 & 64 & 2012.1.00158.S \\
\hline 378 & 2MASS J16230923-2417047 & $161 \pm 1$ & Ophiuchus & 0.13 & 261 & 2013.1.00157.S \\
\hline 379 & $\mathrm{EM} * \mathrm{SR} 21 \mathrm{~A}$ & $138 \pm 1$ & Ophiuchus & 0.23 & 64 & 2012.1.00158.S \\
\hline 380 & [LMW2000] VLA 1623A & $133 * *$ & Ophiuchus & 0.16 & 21 & 2015.1.00084.S \\
\hline 381 & ROXs $12 \mathrm{~B}$ & $133 * *$ & Ophiuchus & 0.15 & 29 & 2015.1.00773.S \\
\hline 382 & ROXs 12B & $133 * *$ & Ophiuchus & 0.66 & 67 & 2016.1.01018.S \\
\hline 383 & DoAr 26 & $133 * *$ & Ophiuchus & 0.30 & 217 & 2015.1.00637.S \\
\hline 384 & $\mathrm{EM} * \mathrm{SR} 24 \mathrm{~B}$ & $133 * *$ & Ophiuchus & 0.20 & 34 & 2013.1.00498.S \\
\hline 385 & BKLT J162736-243020 & $133 * *$ & Ophiuchus & 0.13 & 51 & 2013.1.00100.S \\
\hline 386 & [JJK2008] SMM J162740-24431 †† & $133 * *$ & Ophiuchus & 0.10 & 48 & 2016.1.01042.S \\
\hline 387 & WLY 2-55 & $133 * *$ & Ophiuchus & 0.39 & 100 & 2012.1.00350.S \\
\hline 388 & GBS-VLA J163115.25-243313.8 & $133 * *$ & Ophiuchus & 0.49 & 45 & 2016.1.01018.S \\
\hline 389 & 2MASS J16313124-2426281 & $133 * *$ & Ophiuchus & 0.18 & 19 & 2016.1.00771.S \\
\hline 390 & WSB 71 & $133 * *$ & Ophiuchus & 0.30 & 177 & 2015.1.00637.S \\
\hline 391 & IRAS $16285-2355^{\dagger \dagger}$ & $133 * *$ & Ophiuchus & 0.02 & 16 & 2015.1.01512.S \\
\hline 392 & NAME IRAS 16293-2422B & $133 * *$ & Ophiuchus & 0.20 & 21 & 2013.1.00393.S \\
\hline 393 & $\mathrm{EM} * \mathrm{SR} 24 \mathrm{~S}$ & $114 \pm 5$ & Ophiuchus & 0.14 & 23 & 2013.1.00091.S \\
\hline 394 & V* V1366 Ori & $312 \pm 5$ & Orion & 0.14 & 87 & 2013.1.00658.S \\
\hline 395 & HD 36112 & $160 \pm 2$ & Orion & 0.29 & 87 & 2015.1.01600.S \\
\hline 396 & V* V2377 Ori & $912 \pm 262$ & Orion & 0.43 & 190 & 2011.0.00028.S \\
\hline 397 & [HHM2007] 81 & $376 \pm 37$ & Orion & 0.24 & 281 & 2015.1.00089.S \\
\hline 398 & 2MASS J05375486-0241092 & $393 \pm 19$ & Orion & 0.24 & 281 & 2015.1.00089.S \\
\hline 399 & [BZR99] S Ori 35 & $415 \pm 58$ & Orion & 0.24 & 281 & 2015.1.00089.S \\
\hline 400 & [BZR99] S Ori 12 & $374 \pm 29$ & Orion & 0.24 & 281 & 2015.1.00089.S \\
\hline 401 & Haro 5-7 & $236 \pm 22$ & Orion & 0.24 & 281 & 2015.1.00089.S \\
\hline 402 & 2MASS J05380097-0226079 & $349 \pm 7$ & Orion & 0.24 & 281 & 2015.1.00089.S \\
\hline 403 & 2MASS J05380552-0235571 & $354 \pm 58$ & Orion & 0.24 & 281 & 2015.1.00089.S \\
\hline 404 & V*V1247 Ori & $398 \pm 10$ & Orion & 0.01 & 42 & 2015.1.00986.S \\
\hline 405 & Kiso A-0976 316 & $397 \pm 10$ & Orion & 0.24 & 281 & 2015.1.00089.S \\
\hline 406 & Kiso A-0904 67 & $425 \pm 8$ & Orion & 0.24 & 281 & 2015.1.00089.S \\
\hline 407 & HD 294268 & $374 \pm 7$ & Orion & 0.24 & 281 & 2015.1.00089.S \\
\hline 408 & Kiso A-0976 326 & $392 \pm 11$ & Orion & 0.24 & 281 & 2015.1.00089.S \\
\hline 409 & 2MASS J05382119-0254110 & $344 \pm 16$ & Orion & 0.24 & 281 & 2015.1.00089.S \\
\hline 410 & [W96] rJ053820-0234 & $343 \pm 19$ & Orion & 0.24 & 281 & 2015.1.00089.S \\
\hline 411 & $\mathrm{~V} * \mathrm{~V} 2725$ Ori & $378 \pm 16$ & Orion & 0.24 & 281 & 2015.1.00089.S \\
\hline 412 & V* V505 Ori & $397 \pm 6$ & Orion & 0.24 & 281 & 2015.1.00089.S \\
\hline 413 & V* V2728 Ori & $339 \pm 41$ & Orion & 0.24 & 281 & 2015.1.00089.S \\
\hline 414 & [BNM2013] 93.03227 & $353 \pm 18$ & Orion & 0.24 & 281 & 2015.1.00089.S \\
\hline 415 & Haro 5-9 & $415 \pm 6$ & Orion & 0.24 & 281 & 2015.1.00089.S \\
\hline 416 & $\mathrm{~V} * \mathrm{TX}$ Ori & $572 \pm 85$ & Orion & 0.24 & 281 & 2015.1.00089.S \\
\hline 417 & [BNM2013] 93.0329 & $402 \pm 15$ & Orion & 0.24 & 281 & 2015.1.00089.S \\
\hline 418 & [W96] rJ053833-0236 & $385 \pm 32$ & Orion & 0.24 & 281 & 2015.1.00089.S \\
\hline 419 & 2MASS J05383902-0245321 & $428 \pm 15$ & Orion & 0.24 & 281 & 2015.1.00089.S \\
\hline 420 & 2MASS J05384053-0233275 & $372 \pm 14$ & Orion & 0.24 & 281 & 2015.1.00089.S \\
\hline 421 & [BNM2013] 92.0124 & $388 \pm 12$ & Orion & 0.24 & 281 & 2015.1.00089.S \\
\hline 422 & 2MASS J05384386-0237068 & $385 \pm 13$ & Orion & 0.24 & 281 & 2015.1.00089.S \\
\hline 423 & $\mathrm{~V} * \mathrm{~V} 595$ Ori A & $212 \pm 11$ & Orion & 0.24 & 281 & 2015.1.00089.S \\
\hline 424 & Mayrit 89175 & $395 \pm 20$ & Orion & 0.24 & 281 & 2015.1.00089.S \\
\hline 425 & 2MASS J05384818-0244007 & $428 \pm 33$ & Orion & 0.24 & 281 & 2015.1.00089.S \\
\hline
\end{tabular}


Table A1 - continued

\begin{tabular}{|c|c|c|c|c|c|c|}
\hline $\begin{array}{l}\text { ID } \\
\#\end{array}$ & Name $^{\dagger}$ & $\begin{array}{l}\text { Distance } \\
(\mathrm{pc})\end{array}$ & $\begin{array}{l}\text { Star-forming } \\
\text { region }\end{array}$ & $\begin{array}{c}\text { Resolution } \\
\text { (arcsec) }\end{array}$ & $\begin{array}{c}\text { Sensitivity } \\
\left(\mu \mathrm{Jy}_{\text {beam }}^{-1}\right)\end{array}$ & $\begin{array}{l}\text { ALMA project } \\
\text { code }\end{array}$ \\
\hline 426 & $* *$ CAB 27B & $502 \pm 17$ & Orion & 0.24 & 281 & 2015.1.00089.S \\
\hline 427 & [BHM2009] SigOri-MAD-34 & $404 \pm 8$ & Orion & 0.24 & 281 & 2015.1.00089.S \\
\hline 428 & [W96] pJ053847-0234 & $395 \pm 14$ & Orion & 0.24 & 281 & 2015.1.00089.S \\
\hline 429 & [BZR99] S Ori 15 & $364 \pm 28$ & Orion & 0.24 & 281 & 2015.1.00089.S \\
\hline 430 & 2MASS J05384755-0227120 & $381 \pm 15$ & Orion & 0.24 & 281 & 2015.1.00089.S \\
\hline 431 & 2MASS J05385060-0242429 & $399 \pm 22$ & Orion & 0.24 & 281 & 2015.1.00089.S \\
\hline 432 & 2MASS J05384970-0234526 & $419 \pm 20$ & Orion & 0.24 & 281 & 2015.1.00089.S \\
\hline 433 & $\mathrm{~V} * \mathrm{~V} 2737$ Ori & $412 \pm 52$ & Orion & 0.24 & 281 & 2015.1.00089.S \\
\hline 434 & $\mathrm{~V} * \mathrm{RU}$ Ori & $405 \pm 5$ & Orion & 0.24 & 281 & 2015.1.00089.S \\
\hline 435 & 2MASS J05385831-0216101 & $399 \pm 7$ & Orion & 0.24 & 281 & 2015.1.00089.S \\
\hline 436 & ESO-HA 1693 & $371 \pm 12$ & Orion & 0.24 & 281 & 2015.1.00089.S \\
\hline 437 & 2MASS J05390297-0241272 & $410 \pm 14$ & Orion & 0.24 & 281 & 2015.1.00089.S \\
\hline 438 & 2MASS J05390193-0235029 & $336 \pm 23$ & Orion & 0.24 & 281 & 2015.1.00089.S \\
\hline 439 & Mayrit 458140 & $450 \pm 33$ & Orion & 0.24 & 281 & 2015.1.00089.S \\
\hline 440 & 2MASS J05390387-0220081 & $347 \pm 18$ & Orion & 0.24 & 281 & 2015.1.00089.S \\
\hline 441 & [BZR99] S Ori 7 & $332 \pm 20$ & Orion & 0.24 & 281 & 2015.1.00089.S \\
\hline 442 & 2MASS J05390878-0231115 & $335 \pm 15$ & Orion & 0.24 & 281 & 2015.1.00089.S \\
\hline 443 & [BZR99] S Ori 30 & $339 \pm 57$ & Orion & 0.24 & 281 & 2015.1.00089.S \\
\hline 444 & Haro 5-20 & $411 \pm 7$ & Orion & 0.24 & 281 & 2015.1.00089.S \\
\hline 445 & [BNM2013] 90.02146 & $381 \pm 15$ & Orion & 0.24 & 281 & 2015.1.00089.S \\
\hline 446 & Haro 5-21 & $402 \pm 9$ & Orion & 0.24 & 281 & 2015.1.00089.S \\
\hline 447 & $\mathrm{~V} * \mathrm{BG}$ Ori & $384 \pm 7$ & Orion & 0.24 & 281 & 2015.1.00089.S \\
\hline 448 & 2MASS J05392633-0228376 & $370 \pm 19$ & Orion & 0.24 & 281 & 2015.1.00089.S \\
\hline 449 & 2MASS J05392935-0227209 & $387 \pm 10$ & Orion & 0.24 & 281 & 2015.1.00089.S \\
\hline 450 & Haro $5-25$ & $392 \pm 5$ & Orion & 0.24 & 281 & 2015.1.00089.S \\
\hline 451 & $\mathrm{~V} * \mathrm{RW}$ Ori & $383 \pm 20$ & Orion & 0.24 & 281 & 2015.1.00089.S \\
\hline 452 & V* V603 Ori & $368 \pm 16$ & Orion & 0.24 & 281 & 2015.1.00089.S \\
\hline 453 & Haro 5-27 & $395 \pm 5$ & Orion & 0.24 & 281 & 2015.1.00089.S \\
\hline 454 & $\mathrm{~V} * \mathrm{RV}$ Ori & $398 \pm 4$ & Orion & 0.24 & 281 & 2015.1.00089.S \\
\hline 455 & 2MASS J05394318-0232433 & $363 \pm 13$ & Orion & 0.24 & 281 & 2015.1.00089.S \\
\hline 456 & [BZR99] S Ori 10 & $413 \pm 37$ & Orion & 0.24 & 281 & 2015.1.00089.S \\
\hline 457 & Haro 5-32 & $388 \pm 10$ & Orion & 0.24 & 281 & 2015.1.00089.S \\
\hline 458 & Haro 5-30 & $401 \pm 17$ & Orion & 0.24 & 281 & 2015.1.00089.S \\
\hline 459 & Haro 5-34 & $407 \pm 5$ & Orion & 0.24 & 281 & 2015.1.00089.S \\
\hline 460 & Mayrit 1045094 & $435 \pm 74$ & Orion & 0.24 & 281 & 2015.1.00089.S \\
\hline 461 & 2MASS J05395421-0227326 & $382 \pm 21$ & Orion & 0.24 & 281 & 2015.1.00089.S \\
\hline 462 & V* V605 Ori B & $397 \pm 15$ & Orion & 0.24 & 281 & 2015.1.00089.S \\
\hline 463 & Haro 5-36 & $373 \pm 12$ & Orion & 0.24 & 281 & 2015.1.00089.S \\
\hline 464 & Mayrit 1196092 & $635 \pm 212$ & Orion & 0.24 & 281 & 2015.1.00089.S \\
\hline 465 & 2MASS J05400525-0230522 & $354 \pm 28$ & Orion & 0.24 & 281 & 2015.1.00089.S \\
\hline 466 & $\mathrm{~V} * \mathrm{~V} 608$ Ori & $403 \pm 6$ & Orion & 0.24 & 281 & 2015.1.00089.S \\
\hline 467 & 2MASS J05400933-0225067 & $380 \pm 21$ & Orion & 0.24 & 281 & 2015.1.00089.S \\
\hline 468 & Haro 5-38 & $416 \pm 6$ & Orion & 0.24 & 281 & 2015.1.00089.S \\
\hline 469 & [DRS2009] 2091 & $450 *$ & Orion & 0.43 & 190 & 2011.0.00028.S \\
\hline 470 & $*$ tet01 Ori $\mathrm{F}$ & $450 *$ & Orion & 0.43 & 190 & 2011.0.00028.S \\
\hline 471 & HH $520^{\dagger \dagger}$ & $450 *$ & Orion & 0.07 & 34 & 2017.1.01478.S \\
\hline 472 & [KPM2006] 139 & $450 *$ & Orion & 0.43 & 190 & 2011.0.00028.S \\
\hline 473 & ESO-HA 1656 & $440 * *$ & Orionis & 0.24 & 281 & 2015.1.00089.S \\
\hline 474 & 2MASS J05381189-0245568 & $440 * *$ & Orionis & 0.24 & 281 & 2015.1.00089.S \\
\hline 475 & 2MASS J05381319-0226088 & $440 * *$ & Orionis & 0.24 & 281 & 2015.1.00089.S \\
\hline 476 & 2MASS J05381778-0240500 & $440 * *$ & Orionis & 0.24 & 281 & 2015.1.00089.S \\
\hline 477 & $\mathrm{~V} * \mathrm{~V} 2726$ Ori & $440 * *$ & Orionis & 0.24 & 281 & 2015.1.00089.S \\
\hline 478 & 2XMM J053826.4-023428 & $440 * *$ & Orionis & 0.24 & 281 & 2015.1.00089.S \\
\hline 479 & Mayrit 803197 & $440 * *$ & Orionis & 0.24 & 281 & 2015.1.00089.S \\
\hline 480 & Haro 5-11 & $440 * *$ & Orionis & 0.24 & 281 & 2015.1.00089.S \\
\hline 481 & [W96] rJ053831-0235 & $440 * *$ & Orionis & 0.24 & 281 & 2015.1.00089.S \\
\hline 482 & 2MASS J05383976-0232203 & $440 * *$ & Orionis & 0.24 & 281 & 2015.1.00089.S \\
\hline 483 & [BZR99] S Ori 74 & $440 * *$ & Orionis & 0.24 & 281 & 2015.1.00089.S \\
\hline 484 & [BHM2009] SigOri-MAD-2 & $440 * *$ & Orionis & 0.24 & 281 & 2015.1.00089.S \\
\hline 485 & 2MASS J05385911-0247133 & $440 * *$ & Orionis & 0.24 & 281 & 2015.1.00089.S \\
\hline 486 & GSC2 S02003215312 & $440 * *$ & Orionis & 0.24 & 281 & 2015.1.00089.S \\
\hline 487 & 2MASS J05391427-0221458 & $440 * *$ & Orionis & 0.24 & 281 & 2015.1.00089.S \\
\hline 488 & [HHM2007] 1242 & $440 * *$ & Orionis & 0.24 & 281 & 2015.1.00089.S \\
\hline 489 & 2MASS J05395804-0237402 & $440 * *$ & Orionis & 0.24 & 281 & 2015.1.00089.S \\
\hline 490 & IRAS $03292+3039$ & $300 * *$ & Perseus & 0.15 & 38 & 2015.1.01053.S \\
\hline 491 & 2MASS J17112317-2724315 ${ }^{\dagger \dagger}$ & $130 * *$ & Pipe Nebula & 0.03 & 17 & 2016.1.01186.S \\
\hline 492 & HD 98363 & $139 \pm 1$ & Scorpius-Centaurus Association & 0.67 & 65 & 2015.1.01243.S \\
\hline 493 & HD 131835 & $134 \pm 4$ & Scorpius-Centaurus Association & 0.30 & 40 & 2013.1.01166.S \\
\hline 494 & CPD-36 6759 & $136 \pm 1$ & Scorpius-Centaurus Association & 0.26 & 108 & 2012.1.00870.S \\
\hline 495 & HD 139614 & $135 \pm 1$ & Scorpius-Centaurus Association & 0.54 & 90 & 2015.1.01600.S \\
\hline 496 & HD 141569 & $111 \pm 1$ & Scorpius-Centaurus Association & 0.53 & 115 & 2015.1.01600.S \\
\hline
\end{tabular}


Table A1 - continued

\begin{tabular}{|c|c|c|c|c|c|c|}
\hline $\begin{array}{l}\text { ID } \\
\#\end{array}$ & Name $^{\dagger}$ & $\begin{array}{l}\text { Distance } \\
\text { (pc) }\end{array}$ & $\begin{array}{l}\text { Star-forming } \\
\text { region }\end{array}$ & $\begin{array}{l}\text { Resolution } \\
\text { (arcsec) }\end{array}$ & $\begin{array}{c}\text { Sensitivity } \\
\left(\mu \mathrm{Jy} \mathrm{beam}^{-1}\right)\end{array}$ & $\begin{array}{l}\text { ALMA project } \\
\text { code }\end{array}$ \\
\hline 497 & HD 95086 & $86 \pm 1$ & Scorpius-Centaurus Association & 0.91 & 14 & 2013.1.00773.S \\
\hline 498 & CD-40 8434 & $113 \pm 1$ & Scorpius-Centaurus Association & 0.08 & 30 & 2017.A.00006.S \\
\hline 499 & SH 2-68 N & $193 * *$ & Serpens & 0.51 & 37 & 2015.1.01478.S \\
\hline 500 & [B96] Serpens 2 & $193 * *$ & Serpens & 0.13 & 16 & 2015.1.00354.S \\
\hline 501 & 2MASS J18295766+0113045 & $193 * *$ & Serpens & 0.51 & 37 & 2015.1.01478.S \\
\hline 502 & SSTc2d J182959.5+011159 & $193 * *$ & Serpens & 0.51 & 37 & 2015.1.01478.S \\
\hline 503 & [ETC2005] 19 & $193 * *$ & Serpens & 0.51 & 37 & 2015.1.01478.S \\
\hline 504 & IRAS $04158+2805$ & $90 \pm 5$ & Taurus & 0.06 & 92 & 2016.1.00460.S \\
\hline 505 & HD 32297 & $133 \pm 1$ & Taurus & 0.46 & 21 & 2015.1.00633.S \\
\hline 506 & IRAS $04113+2758$ & $140 * * *$ & Taurus & 0.47 & 103 & 2011.0.00150.S \\
\hline 507 & IP Tau & $131 \pm 1$ & Taurus & 0.09 & 75 & 2016.1.01164.S \\
\hline 508 & MHO 2 & $133 \pm 4$ & Taurus & 0.14 & 51 & 2013.1.00498.S \\
\hline 509 & 2MASS J04141188+2811535 & $131 \pm 3$ & Taurus & 0.25 & 174 & 2012.1.00743.S \\
\hline 510 & $\mathrm{~V} * \mathrm{FM} \mathrm{Tau}$ & $132 \pm 1$ & Taurus & 0.13 & 55 & 2013.1.00426.S \\
\hline 511 & [BCG93] 1 & $136 \pm 2$ & Taurus & 0.42 & 154 & 2011.0.00259.S \\
\hline 512 & $\mathrm{~V} * \mathrm{FP}$ Tau & $128 \pm 1$ & Taurus & 0.23 & 113 & 2012.1.00743.S \\
\hline 513 & $\mathrm{~V} * \mathrm{CX}$ Tau & $128 \pm 1$ & Taurus & 0.13 & 55 & 2013.1.00426.S \\
\hline 514 & $\mathrm{~V} *$ FO Tau & $157 \pm 9$ & Taurus & 0.14 & 90 & 2013.1.00105.S \\
\hline 515 & 2MASS J04161210+2756385 & $137 \pm 2$ & Taurus & 0.23 & 113 & 2012.1.00743.S \\
\hline 516 & $\mathrm{~V} * \mathrm{CY}$ Tau & $129 \pm 1$ & Taurus & 0.14 & 51 & 2013.1.00498.S \\
\hline 517 & 2MASS J04181710+2828419 & $124 \pm 5$ & Taurus & 0.23 & 113 & 2012.1.00743.S \\
\hline 518 & 2MASS J04182147+1658470 & $181 \pm 1$ & Taurus & 0.64 & 150 & 2012.1.00350.S \\
\hline 519 & V*V892 Tau & $117 \pm 2$ & Taurus & 0.14 & 51 & 2013.1.00498.S \\
\hline 520 & 2MASS J04190110+2819420 & $119 \pm 2$ & Taurus & 0.23 & 113 & 2012.1.00743.S \\
\hline 521 & WK81 1 & $132 \pm 1$ & Taurus & 0.64 & 150 & 2012.1.00350.S \\
\hline 522 & 2MASS J04202555+2700355 & $170 \pm 5$ & Taurus & 0.23 & 113 & 2012.1.00743.S \\
\hline 523 & 2MASS J04213459+2701388 & $167 \pm 4$ & Taurus & 0.23 & 113 & 2012.1.00743.S \\
\hline 524 & IRAS $04187+1927$ & $149 \pm 2$ & Taurus & 0.92 & 142 & 2013.1.00105.S \\
\hline 525 & $\mathrm{~V} * \mathrm{DE}$ Tau & $127 \pm 1$ & Taurus & 0.13 & 55 & 2013.1.00426.S \\
\hline 526 & 2MASS J04230607+2801194 & $134 \pm 2$ & Taurus & 0.25 & 174 & 2012.1.00743.S \\
\hline 527 & IRAS $04200+2759$ & $139 \pm 3$ & Taurus & 0.06 & 91 & 2016.1.00460.S \\
\hline 528 & [XCR2012] TrES J042423+265008 & $155 \pm 2$ & Taurus & 0.64 & 150 & 2012.1.00350.S \\
\hline 529 & [BLH2002] KPNO-Tau 3 & $156 \pm 6$ & Taurus & 0.25 & 174 & 2012.1.00743.S \\
\hline 530 & 2MASS J04263055+2443558 & $119 \pm 10$ & Taurus & 0.92 & 142 & 2013.1.00105.S \\
\hline 531 & CoKu FV Tau c & $140 \pm 3$ & Taurus & 0.47 & 159 & 2011.0.00150.S \\
\hline 532 & $\mathrm{~V} * \mathrm{DG} \mathrm{Tau}^{\dagger \dagger}$ & $121 \pm 2$ & Taurus & 0.02 & 12 & 2015.1.01268.S \\
\hline 533 & 2MASS J04295950+2433078 & $131 \pm 3$ & Taurus & 0.14 & 90 & 2013.1.00105.S \\
\hline 534 & $\mathrm{~V} * \mathrm{ZZ}$ Tau & $134 \pm 5$ & Taurus & 0.92 & 142 & 2013.1.00105.S \\
\hline 535 & $\mathrm{~V} *$ HK Tau & $133 \pm 2$ & Taurus & 0.47 & 159 & 2011.0.00150.S \\
\hline 536 & V* V710 Tau B & $145 \pm 1$ & Taurus & 0.14 & 93 & 2013.1.00105.S \\
\hline 537 & Haro 6-13 & $130 \pm 3$ & Taurus & 0.11 & 48 & 2016.1.01042.S \\
\hline 538 & 2MASS J04321606+1812464 & $145 \pm 2$ & Taurus & 0.14 & 93 & 2013.1.00105.S \\
\hline 539 & 2MASS J04322210+1827426 & $142 \pm 2$ & Taurus & 0.23 & 113 & 2012.1.00743.S \\
\hline 540 & 2MASS J04322415+2251083 & $155 \pm 3$ & Taurus & 0.92 & 142 & 2013.1.00105.S \\
\hline 541 & $\mathrm{~V} * \mathrm{FZ} \mathrm{Tau}$ & $130 \pm 1$ & Taurus & 0.13 & 55 & 2013.1.00426.S \\
\hline 542 & JH 112 & $165 \pm 2$ & Taurus & 0.47 & 108 & 2011.0.00150.S \\
\hline 543 & 2MASS J04324938+2253082 & $165 \pm 7$ & Taurus & 0.92 & 142 & 2013.1.00105.S \\
\hline 544 & $\mathrm{~V} * \mathrm{~V} 1321 \mathrm{Tau}$ & $147 \pm 1$ & Taurus & 0.64 & 150 & 2012.1.00350.S \\
\hline 545 & $\mathrm{~V} * \mathrm{~V} 807 \mathrm{Tau}$ & $113 \pm 8$ & Taurus & 0.14 & 90 & 2013.1.00105.S \\
\hline 546 & 2MASS J04330945+2246487 & $149 \pm 9$ & Taurus & 0.92 & 142 & 2013.1.00105.S \\
\hline 547 & IRAS $04303+2240$ & $148 \pm 6$ & Taurus & 0.92 & 142 & 2013.1.00105.S \\
\hline 548 & $\mathrm{~V} *$ GK Tau & $129 \pm 1$ & Taurus & 0.47 & 108 & 2011.0.00150.S \\
\hline 549 & $\mathrm{~V} *$ IS Tau & $133 \pm 5$ & Taurus & 0.14 & 90 & 2013.1.00105.S \\
\hline 550 & $\mathrm{~V} * \mathrm{HN}$ Tau & $137 \pm 3$ & Taurus & 0.47 & 108 & 2011.0.00150.S \\
\hline 551 & $\mathrm{~V} * \mathrm{DL}$ Tau & $159 \pm 1$ & Taurus & 0.20 & 61 & 2015.1.01207.S \\
\hline 552 & 2MASS J04334465+2615005 & $173 \pm 6$ & Taurus & 0.24 & 138 & 2012.1.00743.S \\
\hline 553 & $\mathrm{~V} * \mathrm{DM}$ Tau & $145 \pm 1$ & Taurus & 0.02 & 17 & 2017.1.01460.S \\
\hline 554 & $\mathrm{~V} * \mathrm{CI}$ Tau & $159 \pm 1$ & Taurus & 0.07 & 52 & 2017.A.00014.S \\
\hline 555 & $\mathrm{~V} * \mathrm{AA}$ Tau & $137 \pm 2$ & Taurus & 0.19 & 58 & 2015.1.01017.S \\
\hline 556 & $\mathrm{~V} * \mathrm{HO}$ Tau & $161 \pm 1$ & Taurus & 0.47 & 108 & 2011.0.00150.S \\
\hline 557 & $\mathrm{~V} * \mathrm{DN}$ Tau & $128 \pm 1$ & Taurus & 0.26 & 57 & 2015.1.01207.S \\
\hline 558 & 2MASS J04361030+2159364 & $118 \pm 8$ & Taurus & 0.92 & 142 & 2013.1.00105.S \\
\hline 559 & 2MASS J04362151+2351165 & $115 \pm 2$ & Taurus & 0.92 & 142 & 2013.1.00105.S \\
\hline 560 & HD 283759 & $163 \pm 2$ & Taurus & 0.64 & 150 & 2012.1.00350.S \\
\hline 561 & ITG 3 & $145 \pm 15$ & Taurus & 0.25 & 174 & 2012.1.00743.S \\
\hline 562 & V* GM Tau & $138 \pm 3$ & Taurus & 0.25 & 174 & 2012.1.00743.S \\
\hline 563 & V* DO Tau & $139 \pm 1$ & Taurus & 0.23 & 41 & 2015.1.01207.S \\
\hline 564 & 2MASS J04385859+2336351 & $127 \pm 2$ & Taurus & 0.24 & 138 & 2012.1.00743.S \\
\hline 565 & 2MASS J04390163+2336029 & $128 \pm 1$ & Taurus & 0.24 & 138 & 2012.1.00743.S \\
\hline 566 & 2MASS J04390396+2544264 & $144 \pm 4$ & Taurus & 0.25 & 175 & 2012.1.00743.S \\
\hline 567 & $\mathrm{EM} * \mathrm{LkCa} 15$ & $159 \pm 1$ & Taurus & 0.17 & 68 & 2012.1.00870.S \\
\hline
\end{tabular}


Table A1 - continued

\begin{tabular}{|c|c|c|c|c|c|c|}
\hline $\begin{array}{l}\text { ID } \\
\#\end{array}$ & Name $^{\dagger}$ & $\begin{array}{l}\text { Distance } \\
(\mathrm{pc})\end{array}$ & $\begin{array}{l}\text { Star-forming } \\
\text { region }\end{array}$ & $\begin{array}{c}\text { Resolution } \\
\text { (arcsec) }\end{array}$ & $\begin{array}{c}\text { Sensitivity } \\
\left(\mu \mathrm{Jy} \text { beam }^{-1}\right)\end{array}$ & $\begin{array}{l}\text { ALMA project } \\
\text { code }\end{array}$ \\
\hline 568 & V* VY Tau & $152 \pm 3$ & Taurus & 0.14 & 93 & 2013.1.00105.S \\
\hline 569 & 2MASS J04393364+2359212 & $127 \pm 2$ & Taurus & 0.24 & 138 & 2012.1.00743.S \\
\hline 570 & IRAS F04366+2555 & $137 \pm 2$ & Taurus & 0.24 & 138 & 2012.1.00743.S \\
\hline 571 & 2MASS J04400067+2358211 & $121 \pm 2$ & Taurus & 0.24 & 138 & 2012.1.00743.S \\
\hline 572 & 2MASS J04403979+2519061 & $152 \pm 11$ & Taurus & 0.64 & 150 & 2012.1.00350.S \\
\hline 573 & NAME JH 223B & $141 \pm 3$ & Taurus & 0.14 & 95 & 2013.1.00105.S \\
\hline 574 & ITG 33A & $141 \pm 4$ & Taurus & 0.14 & 95 & 2013.1.00105.S \\
\hline 575 & ITG 34 & $157 \pm 6$ & Taurus & 0.14 & 95 & 2013.1.00105.S \\
\hline 576 & 2MASS J04414489+2301513 & $120 \pm 5$ & Taurus & 0.14 & 95 & 2013.1.00105.S \\
\hline 577 & 2MASS J04414825+2534304 & $136 \pm 4$ & Taurus & 0.25 & 174 & 2012.1.00743.S \\
\hline 578 & V* V999 Tau & $123 \pm 6$ & Taurus & 0.64 & 150 & 2012.1.00350.S \\
\hline 579 & EM* LkHA 332 & $160 \pm 12$ & Taurus & 0.14 & 93 & 2013.1.00105.S \\
\hline 580 & V* GO Tau & $145 \pm 1$ & Taurus & 0.10 & 45 & 2016.1.01164.S \\
\hline 581 & Haro 6-36 & $171 \pm 4$ & Taurus & 0.14 & 95 & 2013.1.00105.S \\
\hline 582 & IRAS S04414+2506 & $141 \pm 3$ & Taurus & 0.42 & 154 & 2011.0.00259.S \\
\hline 583 & $\mathrm{~V} * \mathrm{DR}$ Tau & $196 \pm 2$ & Taurus & 0.16 & 107 & 2016.1.00158.S \\
\hline 584 & $\mathrm{~V} *$ DS Tau & $159 \pm 1$ & Taurus & 0.09 & 44 & 2016.1.01164.S \\
\hline 585 & V*UY Aur & $156 \pm 1$ & Taurus & 0.47 & 105 & 2011.0.00150.S \\
\hline 586 & $\mathrm{~V} * \mathrm{GM}$ Aur & $160 \pm 2$ & Taurus & 0.02 & 15 & 2017.1.01151.S \\
\hline 587 & 2MASS J04554535+3019389 & $155 \pm 2$ & Taurus & 0.14 & 95 & 2013.1.00105.S \\
\hline 588 & $\mathrm{~V} * \mathrm{AB}$ Aur & $163 \pm 2$ & Taurus & 0.14 & 51 & 2012.1.00303.S \\
\hline 589 & 2MASS J04554801+3028050 & $165 \pm 5$ & Taurus & 0.14 & 95 & 2013.1.00105.S \\
\hline 590 & 2MASS J04554969+3019400 & $156 \pm 5$ & Taurus & 0.14 & 95 & 2013.1.00105.S \\
\hline 591 & 2MASS J04560118+3026348 & $159 \pm 9$ & Taurus & 0.14 & 95 & 2013.1.00105.S \\
\hline 592 & $\mathrm{~V} * \mathrm{SU}$ Aur & $158 \pm 1$ & Taurus & 0.13 & 55 & 2013.1.00426.S \\
\hline 593 & HD 31648 & $162 \pm 2$ & Taurus & 0.10 & 45 & 2016.1.01164.S \\
\hline 594 & 2MASS J05062332+2432199 & $151 \pm 10$ & Taurus & 0.14 & 95 & 2013.1.00105.S \\
\hline 595 & 2MASS J05075496+2500156 & $165 \pm 2$ & Taurus & 0.24 & 138 & 2012.1.00743.S \\
\hline 596 & $\mathrm{~V} * \mathrm{CQ}$ Tau & $163 \pm 2$ & Taurus & 0.21 & 31 & 2013.1.00498.S \\
\hline 597 & HD31648 & $162 \pm 2$ & Taurus & 0.11 & 45 & 2016.1.01164.S \\
\hline 598 & LDN 1489 & $140 * *$ & Taurus & 0.65 & 116 & 2011.0.00210.S \\
\hline 599 & $\mathrm{~V} * \mathrm{CZ}$ Tau & $140 * *$ & Taurus & 0.14 & 90 & 2013.1.00105.S \\
\hline 600 & $\mathrm{~V} * \mathrm{FQ}$ Tau & $140 * *$ & Taurus & 0.47 & 159 & 2011.0.00150.S \\
\hline 601 & 2MASS J04202144+2813491 & $140 * *$ & Taurus & 0.06 & 90 & 2016.1.00460.S \\
\hline 602 & 2MASS J04220069+2657324 & $140 * *$ & Taurus & 0.06 & 89 & 2016.1.00460.S \\
\hline 603 & $\mathrm{~V} * \mathrm{FS}$ Tau & $140 * *$ & Taurus & 0.14 & 90 & 2013.1.00105.S \\
\hline 604 & $\mathrm{~V} * \mathrm{FV}$ Tau & $140 * *$ & Taurus & 0.47 & 159 & 2011.0.00150.S \\
\hline 605 & 2MASS J04270266+2605304 & $140 * *$ & Taurus & 0.11 & 44 & 2016.1.01042.S \\
\hline 606 & 2MASS J04284263+2714039 & $140 * *$ & Taurus & 0.23 & 113 & 2012.1.00743.S \\
\hline 607 & IRAS F04262+2654 & $140 * *$ & Taurus & 0.25 & 174 & 2012.1.00743.S \\
\hline 608 & V* DH Tau B & $140 * *$ & Taurus & 0.14 & 30 & 2015.1.00773.S \\
\hline 609 & V* UX Tau & $140 * *$ & Taurus & 0.14 & 93 & 2013.1.00105.S \\
\hline 610 & $\mathrm{~V} * \mathrm{FX}$ Tau & $140 * *$ & Taurus & 0.47 & 159 & 2011.0.00150.S \\
\hline 611 & $\mathrm{~V} * \mathrm{DK}$ Tau & $140 * *$ & Taurus & 0.47 & 103 & 2011.0.00150.S \\
\hline 612 & $\mathrm{~V} * \mathrm{~V} 1213 \mathrm{Tau}^{\dagger \dagger}$ & $140 * *$ & Taurus & 0.02 & 13 & 2017.1.01701.S \\
\hline 613 & $\mathrm{~V} * \mathrm{HL} \mathrm{Tau}^{\dagger \dagger}$ & $140 * *$ & Taurus & 0.09 & 36 & 2013.1.00355.S \\
\hline 614 & $\mathrm{~V} * \mathrm{XZ}$ Tau & $140 * *$ & Taurus & 0.14 & 93 & 2013.1.00105.S \\
\hline 615 & NAME HK Tau B & $140 * *$ & Taurus & 0.06 & 88 & 2016.1.00460.S \\
\hline 616 & $\mathrm{~V} * \mathrm{~V} 710 \mathrm{Tau}$ & $140 * *$ & Taurus & 0.47 & 108 & 2011.0.00150.S \\
\hline 617 & V* GG Tau & $140 * *$ & Taurus & 0.14 & 93 & 2013.1.00105.S \\
\hline 618 & $\mathrm{~V} * \mathrm{GH}$ Tau & $140 * *$ & Taurus & 0.14 & 90 & 2013.1.00105.S \\
\hline 619 & IRAS $04302+2247$ & $140 * *$ & Taurus & 0.06 & 86 & 2016.1.00460.S \\
\hline 620 & $\mathrm{~V} * \mathrm{IT}$ Tau & $140 * *$ & Taurus & 0.47 & 108 & 2011.0.00150.S \\
\hline 621 & NAME CoKu Tau 3 & $140 * *$ & Taurus & 0.47 & 162 & 2011.0.00150.S \\
\hline 622 & Haro 6-28 & $140 * *$ & Taurus & 0.14 & 93 & 2013.1.00105.S \\
\hline 623 & V* HV Tau C & $140 * *$ & Taurus & 0.06 & 91 & 2016.1.00460.S \\
\hline 624 & $\mathrm{~V} * \mathrm{GN}$ Tau & $140 * *$ & Taurus & 0.14 & 93 & 2013.1.00105.S \\
\hline 625 & IRAS $04365+2535$ & $140 * *$ & Taurus & 0.75 & 89 & 2011.0.00210.S \\
\hline 626 & [BCK99] $\mathrm{HCl} 2 \mathrm{NW}-7 \mathrm{a}$ & $140 * *$ & Taurus & 0.42 & 154 & 2011.0.00259.S \\
\hline 627 & LDN 1527 & $140 * *$ & Taurus & 0.75 & 89 & 2011.0.00210.S \\
\hline 628 & ITG 40 & $140 * *$ & Taurus & 0.14 & 95 & 2013.1.00105.S \\
\hline 629 & $\mathrm{~V} * \mathrm{DP}$ Tau & $140 * *$ & Taurus & 0.14 & 93 & 2013.1.00105.S \\
\hline 630 & Haro 6-37 & $140 * *$ & Taurus & 0.14 & 93 & 2013.1.00105.S \\
\hline 631 & 2MASS J04555605+3036209 & $140 * *$ & Taurus & 0.24 & 138 & 2012.1.00743.S \\
\hline 632 & IRAS $05022+2527$ & $140 * *$ & Taurus & 0.47 & 45 & 2011.0.00150.S \\
\hline 633 & HD 282276 & $492 \pm 12 * * *$ & Taurus & 0.64 & 150 & 2012.1.00350.S \\
\hline 634 & $\mathrm{~V} * \mathrm{~V} 1322$ Tau & $463 \pm 13 * * *$ & Taurus & 0.64 & 150 & 2012.1.00350.S \\
\hline 635 & RY Tau & $442 \pm 47 * * *$ & Taurus & 0.02 & 44 & 2017.1.01460.S \\
\hline 636 & 2MASS J10455263-2819303 & $84 \pm 1$ & TW Hya Association & 1.04 & 50 & 2013.1.00457.S \\
\hline 637 & $\mathrm{~V} *$ TW Hya & $60 \pm 1$ & TW Hya Association & 0.04 & 11 & 2017.1.00520.S \\
\hline 638 & 2MASS J11064461-3715115 & $101 \pm 4$ & TW Hya Association & 1.03 & 29 & 2013.1.00457.S \\
\hline
\end{tabular}


Table A1 - continued

\begin{tabular}{|c|c|c|c|c|c|c|}
\hline $\begin{array}{l}\text { ID } \\
\#\end{array}$ & Name $^{\dagger}$ & $\begin{array}{l}\text { Distance } \\
(\mathrm{pc})\end{array}$ & $\begin{array}{l}\text { Star-forming } \\
\text { region }\end{array}$ & $\begin{array}{l}\text { Resolution } \\
(\operatorname{arcsec})\end{array}$ & $\begin{array}{c}\text { Sensitivity } \\
\left(\mu \mathrm{Jy} \mathrm{beam}^{-1}\right)\end{array}$ & $\begin{array}{l}\text { ALMA project } \\
\text { code }\end{array}$ \\
\hline 639 & TWA 37 & $50 \pm 1$ & TW Hya Association & 1.04 & 50 & 2013.1.00457.S \\
\hline 640 & TWA 30 & $48 \pm 1$ & TW Hya Association & 1.04 & 51 & 2013.1.00457.S \\
\hline 641 & TWA 30B & $46 \pm 1$ & TW Hya Association & 1.04 & 51 & 2013.1.00457.S \\
\hline 642 & TWA 33 & $49 \pm 1$ & TW Hya Association & 1.03 & 29 & 2013.1.00457.S \\
\hline 643 & TWA 38 & $80 \pm 1$ & TW Hya Association & 0.99 & 39 & 2013.1.00457.S \\
\hline 644 & TWA 31 & $81 \pm 1$ & TW Hya Association & 1.04 & 51 & 2013.1.00457.S \\
\hline 645 & TWA 27 & $64 \pm 1$ & TW Hya Association & 0.28 & 36 & 2013.1.01016.S \\
\hline 646 & TWA 40 & $67 \pm 4$ & TW Hya Association & 0.99 & 39 & 2013.1.00457.S \\
\hline 647 & HD 109573 & $72 \pm 1$ & TW Hya Association & 0.17 & 39 & 2015.1.00032.S \\
\hline 648 & HD 109832 & $108 \pm 1$ & TW Hya Association & 0.67 & 60 & 2015.1.01243.S \\
\hline 649 & TWA 29 & $83 \pm 3$ & TW Hya Association & 0.92 & 39 & 2013.1.00457.S \\
\hline 650 & 2MASS J12474428-3816464 & $85 \pm 3$ & TW Hya Association & 0.99 & 39 & 2013.1.00457.S \\
\hline 651 & 2MASS J12520989-4948280 & $116 \pm 4$ & TW Hya Association & 0.92 & 39 & 2013.1.00457.S \\
\hline 652 & 2MASS J13265348-5022270 & $107 \pm 7$ & TW Hya Association & 0.92 & 39 & 2013.1.00457.S \\
\hline 653 & EC $13436-1335$ & $88 \pm 1$ & TW Hya Association & 0.69 & 43 & 2015.1.00783.S \\
\hline 654 & HE $1350-1612$ & $109 \pm 2$ & TW Hya Association & 0.69 & 43 & 2015.1.00783.S \\
\hline 655 & TWA 32 & $50 * *$ & TW Hya Association & 0.99 & 39 & 2013.1.00457.S \\
\hline 656 & HD $98800^{\dagger \dagger}$ & $50 * *$ & TW Hya Association & 0.10 & 33 & 2016.1.01042.S \\
\hline 657 & TWA 34 & $61 \pm 1$ & TW Hya Association & 1.04 & 50 & 2013.1.00457.S \\
\hline 658 & V* V419 Нуа & $22 \pm 1$ & TW Hya Association & 0.40 & 29 & 2016.1.00104.S \\
\hline 659 & $\mathrm{~V} * \mathrm{CE} \mathrm{Ant}^{\dagger \dagger}$ & $34 \pm 1$ & TW Hya Association & 0.10 & 33 & 2015.1.01015.S \\
\hline 660 & * eps Eri & $3 \pm 1$ & Ursa Major Moving Group & 1.04 & 30 & 2013.1.00645.S \\
\hline 661 & Haro 1-5 & $140 * *$ & Upper Scorpius & 0.30 & 172 & 2015.1.00637.S \\
\hline 662 & UCAC2 23646111 & $136 \pm 3$ & Upper Scorpius & 0.30 & 204 & 2013.1.00395.S \\
\hline 663 & ScoPMS 8b & $125 \pm 2$ & Upper Scorpius & 0.28 & 187 & 2013.1.00395.S \\
\hline 664 & HD 142506 & $144 \pm 1$ & Upper Scorpius & 0.28 & 186 & 2013.1.00395.S \\
\hline 665 & DENIS J155556.0-204518 & $146 \pm 5$ & Upper Scorpius & 0.37 & 161 & 2012.1.00743.S \\
\hline 666 & UScoCTIO 113 & $140 \pm 5$ & Upper Scorpius & 0.37 & 161 & 2012.1.00743.S \\
\hline 667 & [PBB2002] USco J155624.8-222555 & $141 \pm 2$ & Upper Scorpius & 0.46 & 392 & 2011.0.00526.S \\
\hline 668 & HD 142666 & $148 \pm 1$ & Upper Scorpius & 0.20 & 34 & 2013.1.00498.S \\
\hline 669 & [PBB2002] USco J155706.4-220606 & $158 \pm 3$ & Upper Scorpius & 0.46 & 254 & 2011.0.00526.S \\
\hline 670 & CD-23 12602 & $143 \pm 1$ & Upper Scorpius & 0.28 & 186 & 2013.1.00395.S \\
\hline 671 & RX J155829.5-231026 & $148 \pm 3$ & Upper Scorpius & 0.46 & 392 & 2011.0.00526.S \\
\hline 672 & HD 143006 & $166 \pm 4$ & Upper Scorpius & 0.40 & 62 & 2015.1.00964.S \\
\hline 673 & 2MASS J15584772-1757595 & $139 \pm 1$ & Upper Scorpius & 0.30 & 204 & 2013.1.00395.S \\
\hline 674 & UScoCTIO 128 & $140 \pm 7$ & Upper Scorpius & 0.37 & 161 & 2012.1.00743.S \\
\hline 675 & 2MASS J16001330-2418106 & $146 \pm 1$ & Upper Scorpius & 0.28 & 186 & 2013.1.00395.S \\
\hline 676 & UCAC3 135-174588 & $149 \pm 2$ & Upper Scorpius & 0.28 & 186 & 2013.1.00395.S \\
\hline 677 & [PBB2002] USco J160018.4-223011 & $138 \pm 8$ & Upper Scorpius & 0.28 & 186 & 2013.1.00395.S \\
\hline 678 & 2MASS J16014157-2111380 & $145 \pm 2$ & Upper Scorpius & 0.30 & 204 & 2013.1.00395.S \\
\hline 679 & RX J1602.0-2221 & $145 \pm 2$ & Upper Scorpius & 0.28 & 186 & 2013.1.00395.S \\
\hline 680 & [PBB2002] USco J160207.5-225746 & $140 \pm 1$ & Upper Scorpius & 0.28 & 186 & 2013.1.00395.S \\
\hline 681 & EPIC 204607034 & $142 \pm 3$ & Upper Scorpius & 0.29 & 188 & 2013.1.00395.S \\
\hline 682 & UScoCTIO 45 & $96 \pm 3$ & Upper Scorpius & 0.37 & 161 & 2012.1.00743.S \\
\hline 683 & RX J1602.8-2401B & $144 \pm 1$ & Upper Scorpius & 0.28 & 186 & 2013.1.00395.S \\
\hline 684 & UScoCTIO 59 & $144 \pm 3$ & Upper Scorpius & 0.29 & 188 & 2013.1.00395.S \\
\hline 685 & 2MASS J16031329-2112569 & $143 \pm 2$ & Upper Scorpius & 0.29 & 188 & 2013.1.00395.S \\
\hline 686 & 2MASS J16032225-2413111 & $144 \pm 3$ & Upper Scorpius & 0.28 & 186 & 2013.1.00395.S \\
\hline 687 & 2MASS J16035767-2031055 & $143 \pm 1$ & Upper Scorpius & 0.50 & 246 & 2011.0.00526.S \\
\hline 688 & [PGZ2001] J160357.9-194210 & $158 \pm 2$ & Upper Scorpius & 0.30 & 204 & 2013.1.00395.S \\
\hline 689 & EPIC 205037578 & $161 \pm 2$ & Upper Scorpius & 0.30 & 204 & 2013.1.00395.S \\
\hline 690 & 2MASS J16042165-2130284 & $150 \pm 1$ & Upper Scorpius & 0.16 & 46 & 2015.1.00888.S \\
\hline 691 & [PGZ2001] J160439.1-194245 & $151 \pm 2$ & Upper Scorpius & 0.30 & 204 & 2013.1.00395.S \\
\hline 692 & 2MASS J16050231-1941554 & $158 \pm 3$ & Upper Scorpius & 0.30 & 204 & 2013.1.00395.S \\
\hline 693 & 2MASS J16052459-1954419 & $153 \pm 2$ & Upper Scorpius & 0.30 & 204 & 2013.1.00395.S \\
\hline 694 & [PGZ2001] J160525.5-203539 & $143 \pm 3$ & Upper Scorpius & 0.46 & 254 & 2011.0.00526.S \\
\hline 695 & [PGZ2001] J160532.1-193315 & $154 \pm 3$ & Upper Scorpius & 0.46 & 254 & 2011.0.00526.S \\
\hline 696 & [PGZ2001] J160545.4-202308 & $145 \pm 2$ & Upper Scorpius & 0.30 & 204 & 2013.1.00395.S \\
\hline 697 & 2MASS J16055863-1949029 & $149 \pm 2$ & Upper Scorpius & 0.30 & 204 & 2013.1.00395.S \\
\hline 698 & DENIS J160603.9-205644 & $137 \pm 5$ & Upper Scorpius & 0.37 & 161 & 2012.1.00743.S \\
\hline 699 & 2MASS J16061330-2212537 & $140 \pm 2$ & Upper Scorpius & 0.29 & 188 & 2013.1.00395.S \\
\hline 700 & [PGZ2001] J160622.8-201124 & $151 \pm 2$ & Upper Scorpius & 0.43 & 135 & 2011.0.00526.S \\
\hline 701 & 2MASS J16063539-2516510 & $139 \pm 3$ & Upper Scorpius & 0.28 & 186 & 2013.1.00395.S \\
\hline 702 & 2MASS J16064115-2517044 & $149 \pm 2$ & Upper Scorpius & 0.28 & 186 & 2013.1.00395.S \\
\hline 703 & 2MASS J16064102-2455489 & $152 \pm 3$ & Upper Scorpius & 0.28 & 186 & 2013.1.00395.S \\
\hline 704 & [PGZ2001] J160643.8-190805 & $144 \pm 7$ & Upper Scorpius & 0.50 & 246 & 2011.0.00526.S \\
\hline 705 & [PGZ2001] J160700.1-203309 & $139 \pm 2$ & Upper Scorpius & 0.30 & 199 & 2013.1.00395.S \\
\hline 706 & 1RXS J160708.6-192737 & $146 \pm 2$ & Upper Scorpius & 0.30 & 199 & 2013.1.00395.S \\
\hline 707 & [PGZ2001] J160719.7-202055 & $164 \pm 3$ & Upper Scorpius & 0.30 & 199 & 2013.1.00395.S \\
\hline 708 & EPIC 203889938 & $143 \pm 2$ & Upper Scorpius & 0.28 & 186 & 2013.1.00395.S \\
\hline 709 & [PGZ2001] J160739.4-191747 & $137 \pm 1$ & Upper Scorpius & 0.30 & 199 & 2013.1.00395.S \\
\hline
\end{tabular}


Table A1 - continued

\begin{tabular}{|c|c|c|c|c|c|c|}
\hline $\begin{array}{l}\text { ID } \\
\#\end{array}$ & Name $^{\dagger}$ & $\begin{array}{l}\text { Distance } \\
(\mathrm{pc})\end{array}$ & $\begin{array}{l}\text { Star-forming } \\
\text { region }\end{array}$ & $\begin{array}{l}\text { Resolution } \\
(\operatorname{arcsec})\end{array}$ & $\begin{array}{c}\text { Sensitivity } \\
\left(\mu \mathrm{Jy} \mathrm{beam}^{-1}\right)\end{array}$ & $\begin{array}{l}\text { ALMA project } \\
\text { code }\end{array}$ \\
\hline 710 & EPIC 204830786 & $198 \pm 8$ & Upper Scorpius & 0.29 & 188 & 2013.1.00395.S \\
\hline 711 & 2MASS J16080555-2218070 & $143 \pm 1$ & Upper Scorpius & 0.29 & 188 & 2013.1.00395.S \\
\hline 712 & 2MASS J16081566-2222199 & $140 \pm 2$ & Upper Scorpius & 0.29 & 188 & 2013.1.00395.S \\
\hline 713 & [T64] 3 & $138 \pm 1$ & Upper Scorpius & 0.50 & 246 & 2011.0.00526.S \\
\hline 714 & 2MASS J16083455-2211559 & $136 \pm 3$ & Upper Scorpius & 0.29 & 188 & 2013.1.00395.S \\
\hline 715 & 2MASS J16084894-2400045 & $145 \pm 2$ & Upper Scorpius & 0.28 & 186 & 2013.1.00395.S \\
\hline 716 & [PGZ2001] J160900.7-190852 & $138 \pm 1$ & Upper Scorpius & 0.43 & 135 & 2011.0.00526.S \\
\hline 717 & [PBB2002] USco J160900.0-190836 & $139 \pm 3$ & Upper Scorpius & 0.43 & 135 & 2011.0.00526.S \\
\hline 718 & [PBB2002] USco J160935.6-182822 & $165 \pm 3$ & Upper Scorpius & 0.30 & 199 & 2013.1.00395.S \\
\hline 719 & 2MASS J16094098-2217594 & $146 \pm 1$ & Upper Scorpius & 0.29 & 188 & 2013.1.00395.S \\
\hline 720 & [PBB2002] USco J160953.6-175446 & $158 \pm 5$ & Upper Scorpius & 0.30 & 199 & 2013.1.00395.S \\
\hline 721 & [PGZ2001] J160954.4-190654 & $137 \pm 1$ & Upper Scorpius & 0.30 & 199 & 2013.1.00395.S \\
\hline 722 & [PGZ2001] J160959.4-180009 & $136 \pm 2$ & Upper Scorpius & 0.46 & 392 & 2011.0.00526.S \\
\hline 723 & DENIS J161005.4-191936 & $150 \pm 7$ & Upper Scorpius & 0.37 & 161 & 2012.1.00743.S \\
\hline 724 & $\mathrm{~K} 2-33$ & $140 \pm 2$ & Upper Scorpius & 0.30 & 199 & 2013.1.00395.S \\
\hline 725 & EPIC 203756600 & $156 \pm 4$ & Upper Scorpius & 0.28 & 186 & 2013.1.00395.S \\
\hline 726 & ScoPMS 42b & $134 \pm 1$ & Upper Scorpius & 0.30 & 199 & 2013.1.00395.S \\
\hline 727 & [PBB2002] USco J161028.1-191043 & $151 \pm 3$ & Upper Scorpius & 0.30 & 199 & 2013.1.00395.S \\
\hline 728 & [PGZ2001] J161039.5-191652 & $159 \pm 2$ & Upper Scorpius & 0.30 & 199 & 2013.1.00395.S \\
\hline 729 & GSC 06213-01459 & $140 \pm 1$ & Upper Scorpius & 0.29 & 188 & 2013.1.00395.S \\
\hline 730 & 2MASS J16104636-1840598 & $143 \pm 3$ & Upper Scorpius & 0.30 & 199 & 2013.1.00395.S \\
\hline 731 & UCAC2 23893922 & $155 \pm 2$ & Upper Scorpius & 0.30 & 199 & 2013.1.00395.S \\
\hline 732 & 1RXS J161115.1-175741 & $137 \pm 1$ & Upper Scorpius & 0.30 & 199 & 2013.1.00395.S \\
\hline 733 & ScoPMS 45 & $137 \pm 1$ & Upper Scorpius & 0.30 & 199 & 2013.1.00395.S \\
\hline 734 & 2MASS J16115091-2012098 & $152 \pm 4$ & Upper Scorpius & 0.30 & 199 & 2013.1.00395.S \\
\hline 735 & 2MASS J16122737-2009596 & $147 \pm 4$ & Upper Scorpius & 0.30 & 199 & 2013.1.00395.S \\
\hline 736 & [T64] 6 & $139 \pm 2$ & Upper Scorpius & 0.30 & 199 & 2013.1.00395.S \\
\hline 737 & [PBB2002] USco J161248.9-180052 & $158 \pm 2$ & Upper Scorpius & 0.30 & 199 & 2013.1.00395.S \\
\hline 738 & HD 145655 & $152 \pm 1$ & Upper Scorpius & 0.29 & 188 & 2013.1.00395.S \\
\hline 739 & EPIC 205165965 & $137 \pm 2$ & Upper Scorpius & 0.30 & 199 & 2013.1.00395.S \\
\hline 740 & HD 145718 & $153 \pm 2$ & Upper Scorpius & 0.72 & 79 & 2015.1.01600.S \\
\hline 741 & [T64] 7 & $143 \pm 3$ & Upper Scorpius & 0.43 & 135 & 2011.0.00526.S \\
\hline 742 & EPIC 205188906 & $141 \pm 2$ & Upper Scorpius & 0.30 & 199 & 2013.1.00395.S \\
\hline 743 & [PGZ2001] J161433.6-190013 & $142 \pm 2$ & Upper Scorpius & 0.30 & 199 & 2013.1.00395.S \\
\hline 744 & RX J1614.6-1858 & $101 \pm 1$ & Upper Scorpius & 0.39 & 100 & 2012.1.00350.S \\
\hline 745 & 2MASS J16145918-2750230 & $149 \pm 1$ & Upper Scorpius & 0.28 & 186 & 2013.1.00395.S \\
\hline 746 & EPIC 203770673 & $159 \pm 3$ & Upper Scorpius & 0.29 & 188 & 2013.1.00395.S \\
\hline 747 & EPIC 203938167 & $154 \pm 3$ & Upper Scorpius & 0.29 & 188 & 2013.1.00395.S \\
\hline 748 & GSC 06209-00747 & $132 \pm 2$ & Upper Scorpius & 0.30 & 199 & 2013.1.00395.S \\
\hline 749 & EPIC 203664569 & $163 \pm 1$ & Upper Scorpius & 0.29 & 188 & 2013.1.00395.S \\
\hline 750 & 2MASS J16181904-2028479 & $138 \pm 2$ & Upper Scorpius & 0.29 & 188 & 2013.1.00395.S \\
\hline 751 & 2MASS J16214199-2313432 & $139 \pm 2$ & Upper Scorpius & 0.37 & 161 & 2012.1.00743.S \\
\hline 752 & GSC $06214-00210$ & $109 \pm 1$ & Upper Scorpius & 0.29 & 188 & 2013.1.00395.S \\
\hline 753 & 2MASS J16220961-1953005 & $138 \pm 2$ & Upper Scorpius & 0.64 & 152 & 2011.0.00733.S \\
\hline 754 & 2MASS J16223757-2345508 & $137 \pm 1$ & Upper Scorpius & 0.39 & 100 & 2012.1.00350.S \\
\hline 755 & CD-22 11523 & $140 \pm 1$ & Upper Scorpius & 0.29 & 188 & 2013.1.00395.S \\
\hline 756 & HD 147594 & $134 \pm 1$ & Upper Scorpius & 0.29 & 188 & 2013.1.00395.S \\
\hline 757 & 2MASS J16251469-2456069 & $136 \pm 1$ & Upper Scorpius & 0.39 & 100 & 2012.1.00350.S \\
\hline 758 & GSS 31 & $138 \pm 2$ & Upper Scorpius & 0.30 & 177 & 2015.1.00637.S \\
\hline 759 & BKLT J162620-240854 & $136 \pm 1$ & Upper Scorpius & 0.30 & 217 & 2015.1.00637.S \\
\hline 760 & Elia 2-24 & $136 \pm 2$ & Upper Scorpius & 0.20 & 34 & 2013.1.00498.S \\
\hline 761 & GSS 39 & $116 \pm 13$ & Upper Scorpius & 0.20 & 34 & 2013.1.00498.S \\
\hline 762 & [GY92] 204 & $142 \pm 3$ & Upper Scorpius & 0.42 & 295 & 2011.0.00259.S \\
\hline 763 & 2MASS J16270942-2148457 & $140 \pm 3$ & Upper Scorpius & 0.29 & 188 & 2013.1.00395.S \\
\hline 764 & YLW 58 & $137 \pm 3$ & Upper Scorpius & 0.10 & 48 & 2016.1.01042.S \\
\hline 765 & 2MASS J16303390-2428062 & $151 \pm 3$ & Upper Scorpius & 0.29 & 188 & 2013.1.00395.S \\
\hline 766 & HD 163296 & $101 \pm 1$ & Upper Scorpius & 0.17 & 138 & 2015.1.00847.S \\
\hline 767 & DoAr 25 & $138 \pm 1$ & Upper Scorpius & 0.03 & 18 & 2016.1.00484.L \\
\hline 768 & Elia $2-20^{\dagger \dagger}$ & $138 \pm 4$ & Upper Scorpius & 0.02 & 15 & 2016.1.00484.L \\
\hline 769 & $\mathrm{EM} * \mathrm{SR} 4$ & $135 \pm 1$ & Upper Scorpius & 0.02 & 15 & 2016.1.00484.L \\
\hline 770 & V* V1094 Sco & $154 \pm 1$ & Upper Scorpius & 0.24 & 61 & 2016.1.01239.S \\
\hline 771 & UScoCTIO 13 & $140 * *$ & Upper Scorpius & 0.46 & 392 & 2011.0.00526.S \\
\hline 772 & [PBB2002] USco J160140.8-225810 & $140 * *$ & Upper Scorpius & 0.28 & 186 & 2013.1.00395.S \\
\hline 773 & [PBB2002] USco J160202.9-223613 & $140 * *$ & Upper Scorpius & 0.28 & 186 & 2013.1.00395.S \\
\hline 774 & 2MASS J16052661-1957050 & $140 * *$ & Upper Scorpius & 0.30 & 204 & 2013.1.00395.S \\
\hline 775 & [PGZ2001] J160600.6-195711 & $140 * *$ & Upper Scorpius & 0.43 & 135 & 2011.0.00526.S \\
\hline 776 & ScoPMS 31 & $140 * *$ & Upper Scorpius & 0.50 & 246 & 2011.0.00526.S \\
\hline 777 & [PGZ2001] J160702.1-201938 & $140 * *$ & Upper Scorpius & 0.46 & 254 & 2011.0.00526.S \\
\hline 778 & 2MASS J16072747-2059442 & $140 * *$ & Upper Scorpius & 0.29 & 188 & 2013.1.00395.S \\
\hline 779 & [PGZ2001] J160827.5-194904 & $140 * *$ & Upper Scorpius & 0.43 & 135 & 2011.0.00526.S \\
\hline 780 & RX J1609.5-2105B & $140 * *$ & Upper Scorpius & 0.15 & 29 & 2015.1.00773.S \\
\hline
\end{tabular}


Table A1 - continued

\begin{tabular}{|c|c|c|c|c|c|c|}
\hline $\begin{array}{l}\text { ID } \\
\#\end{array}$ & Name $^{\dagger}$ & $\begin{array}{c}\text { Distance } \\
(\mathrm{pc})\end{array}$ & $\begin{array}{l}\text { Star-forming } \\
\text { region }\end{array}$ & $\begin{array}{l}\text { Resolution } \\
(\operatorname{arcsec})\end{array}$ & $\begin{array}{c}\text { Sensitivity } \\
\left(\mu \mathrm{Jy} \text { beam }^{-1}\right)\end{array}$ & $\begin{array}{l}\text { ALMA project } \\
\text { code }\end{array}$ \\
\hline 781 & ScoPMS 42a & $140 * *$ & Upper Scorpius & 0.30 & 199 & 2013.1.00395.S \\
\hline 782 & $\mathrm{~V} * \mathrm{~V} 866 \mathrm{Sco}$ & $140 * *$ & Upper Scorpius & 0.52 & 305 & 2011.0.00531.S \\
\hline 783 & UCAC2 24372422 & $140 * *$ & Upper Scorpius & 0.39 & 100 & 2012.1.00350.S \\
\hline 784 & EPIC 203750883 & $140 * *$ & Upper Scorpius & 0.28 & 186 & 2013.1.00395.S \\
\hline 785 & 2MASS J16135434-2320342 & $140 * *$ & Upper Scorpius & 0.29 & 188 & 2013.1.00395.S \\
\hline 786 & CD-22 11432 & $140 * *$ & Upper Scorpius & 0.43 & 135 & 2011.0.00526.S \\
\hline 787 & $\mathrm{~V} * \mathrm{VV}$ Sco & $140 * *$ & Upper Scorpius & 0.29 & 188 & 2013.1.00395.S \\
\hline 788 & DENIS J161816.2-261908 & $140 * *$ & Upper Scorpius & 0.29 & 188 & 2013.1.00395.S \\
\hline 789 & WSB 4 & $140 * *$ & Upper Scorpius & 0.30 & 217 & 2015.1.00637.S \\
\hline 790 & DENIS J161939.8-214535 & $140 * *$ & Upper Scorpius & 0.37 & 161 & 2012.1.00743.S \\
\hline 791 & WSB 18 & $140 * *$ & Upper Scorpius & 0.30 & 221 & 2015.1.00637.S \\
\hline 792 & GSS $26^{\dagger \dagger}$ & $140 * *$ & Upper Scorpius & 0.10 & 48 & 2016.1.01042.S \\
\hline 793 & * zet02 Ret & $12 \pm 1$ & $\zeta$ Herculis Moving Group & 2.74 & 167 & 2017.1.00786.S \\
\hline
\end{tabular}

Notes.

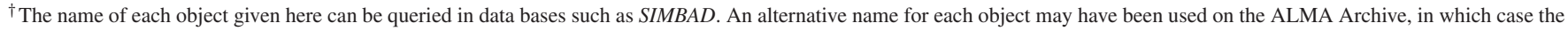
project code should also be used to find the object.

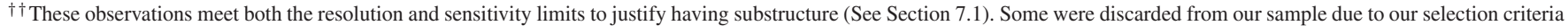
(see Section 2).

*These sources have no associated Gaia distances and their distance was obtained from literature measurements. V* RW Aur, HD 98800, * Alf PsA and $*$ Bet Leo (van Leeuwen 2007), EM* AS 220 (Gaia Collaboration 2016), 2MASS J18191220-2047297 (Maud et al. 2015), IRAS 13481-6124 (Fontani et al. 2005).

**These sources have no associated Gaia distance, nor individual distance estimate. Therefore, we approximate the distance by using the distance to its association.

***These sources have been attributed to belonging to the associated star-forming region. The updated Gaia distances, however, put this into question.

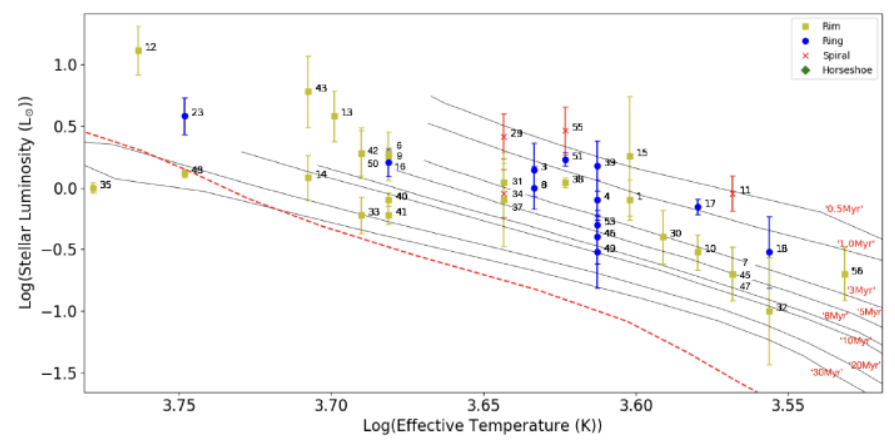

Figure B1. An HR diagram of the low-mass $\left(<1.4 M_{\odot}\right)$ sources studied in this work. The red dashed line marks the ZAMS using the model of Siess et al. (2000). Stellar tracks for a range of ages have been overplotted as black lines from the models of Baraffe et al. (2015). The discs have been labelled and their identities can be found in Table 1.

\section{APPENDIX B: STELLAR MASS AND AGE DETERMINATIONS}

Figs B1 to B4 display the HR diagram shown in Fig. 4 with the addition of stellar tracks from the models of Baraffe et al. (2015) and Siess et al. (2000). These evolutionary diagrams have been used to

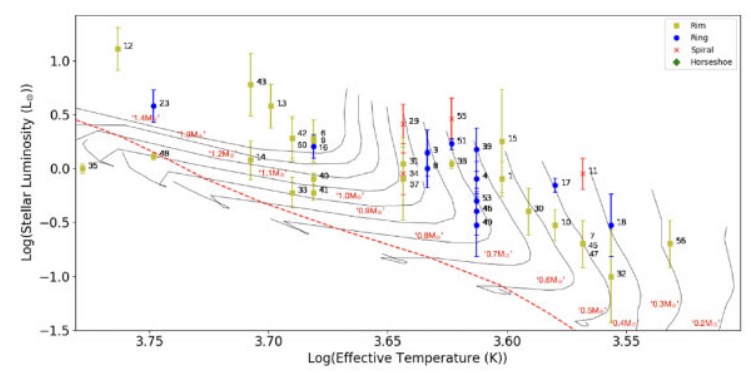

Figure B2. An HR diagram of the low-mass $\left(<1.4 M_{\odot}\right)$ sources studied in this work. The red dashed line marks the ZAMS using the model of Siess et al. (2000). Stellar tracks for a range of masses have been overplotted as black lines from the models of Baraffe et al. (2015). The discs have been labelled and their identities can be found in Table 1. determine the stellar masses and ages of the sources in our sample, the values of which can be found in Tables 1 and 2, respectively. The following plots and tables can be found online.

We compare the derived ages to the average age of the star-forming region each object belongs to. The ages of each star-forming region can be found online in Table B1.

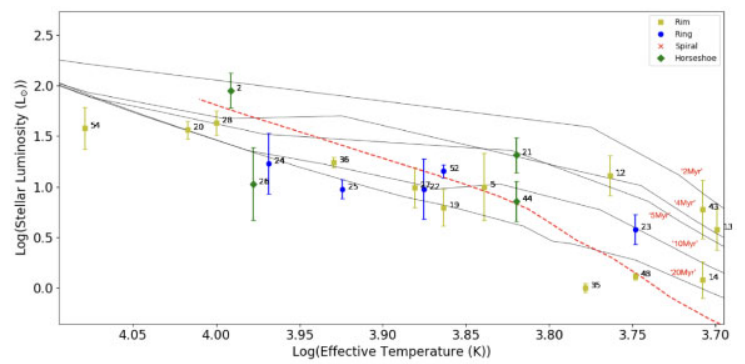

Figure B3. An HR diagram of the high-mass $\left(>1.4 M_{\odot}\right)$ sources studied in this work. The red dashed line marks the ZAMS using the model of Siess et al. (2000). Stellar tracks for a range of ages have been overplotted as black lines from the models of Siess et al. (2000). The discs have been labelled and their identities can be found in Table 1 . 


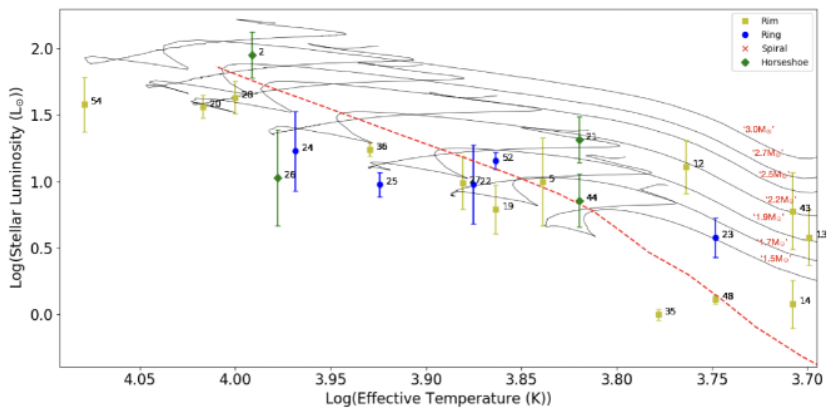

Figure B4. An HR diagram of the high-mass $\left(>1.4 M_{\odot}\right)$ sources studied in this work. The red dashed line marks the ZAMS using the model of Siess et al. (2000). Stellar tracks for a range of masses have been overplotted as black lines from the models of Siess et al. (2000). The discs have been labelled and their identities can be found in Table 1 .
Table B1. Average age of nearby star-forming regions used to compare the ages derived in Section 4.1.

\begin{tabular}{lcc}
\hline SFR & Age $(\mathrm{Myr})$ & Reference \\
\hline Beta Pic & $22 \pm 3$ & $(1)$ \\
Chamaeleon & $3-4$ & $(2)$ \\
Corona Australis & $9 \pm 4$ & $(3)$ \\
Lower Centaurus Crux & 16 & $(4)$ \\
Lupus & 3 & $(5)$ \\
Ophiuchus & $2-5$ & $(6)$ \\
Orion A & $1-3$ & $(7)$ \\
Orion OB1 & 5 & $(8)$ \\
Taurus & $1-5$ & $(9)$ \\
TW Hya & $10 \pm 3$ & $(10)$ \\
Upper Scorpius & 5 & $(4)$
\end{tabular}

Note. References: (1) Mamajek \& Bell (2014), (2) Luhman (2007), (3) James et al. (2006), (4) Preibisch \& Mamajek (2008), (5) Alcalá et al. (2017), (6) Wilking, Gagné \& Allen (2008), (7) Da Rio et al. (2010), (8) Caballero \& Solano (2008), (9) Kraus \& Hillenbrand (2009), (10) Bell, Mamajek \& Naylor (2015)

\section{APPENDIX C: CATALOGUE OF PROTOPLANETARY DISCS STUDIED}

Figs $\mathrm{C} 1$ to $\mathrm{C} 4$ display the full catalogues of discs investigated in this work. Figs $\mathrm{C} 1$ to $\mathrm{C} 4$, respectively, show the rim, ring, horseshoe, and spiral class discs, with the discs within each class ordered by their stellar age. These images were discussed in Section 6.2. All figures in Appendix $\mathrm{C}$ can be found online.

Figs $\mathrm{C} 5$ to $\mathrm{C} 8$, respectively, show the rim, ring, horseshoe, and spiral class discs, with the discs within each class ordered by their stellar mass. 

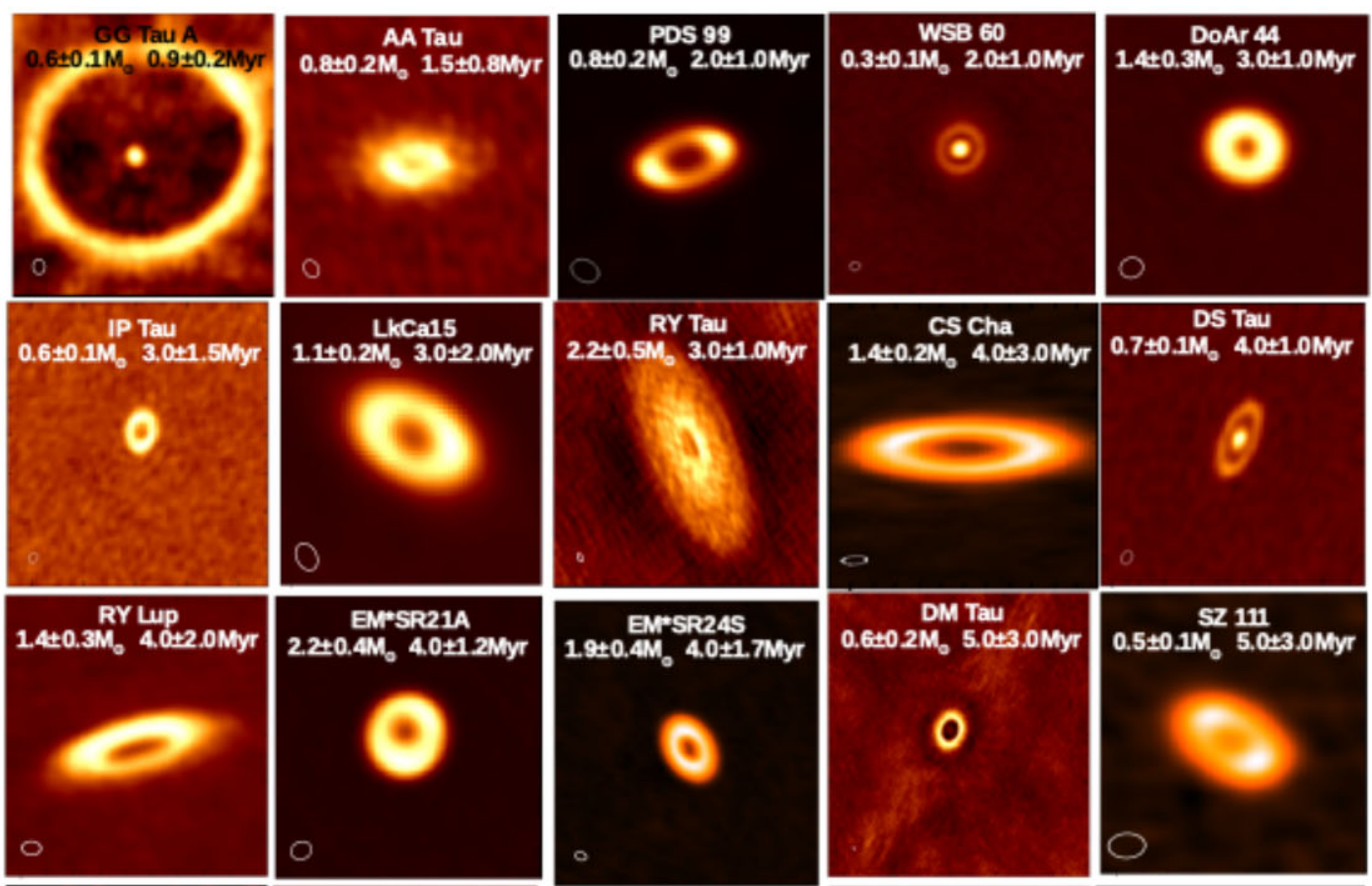

$0.7 \pm 0.1 \mathrm{M}_{6} \quad 4.0 \pm 1.0 \mathrm{Myr}$
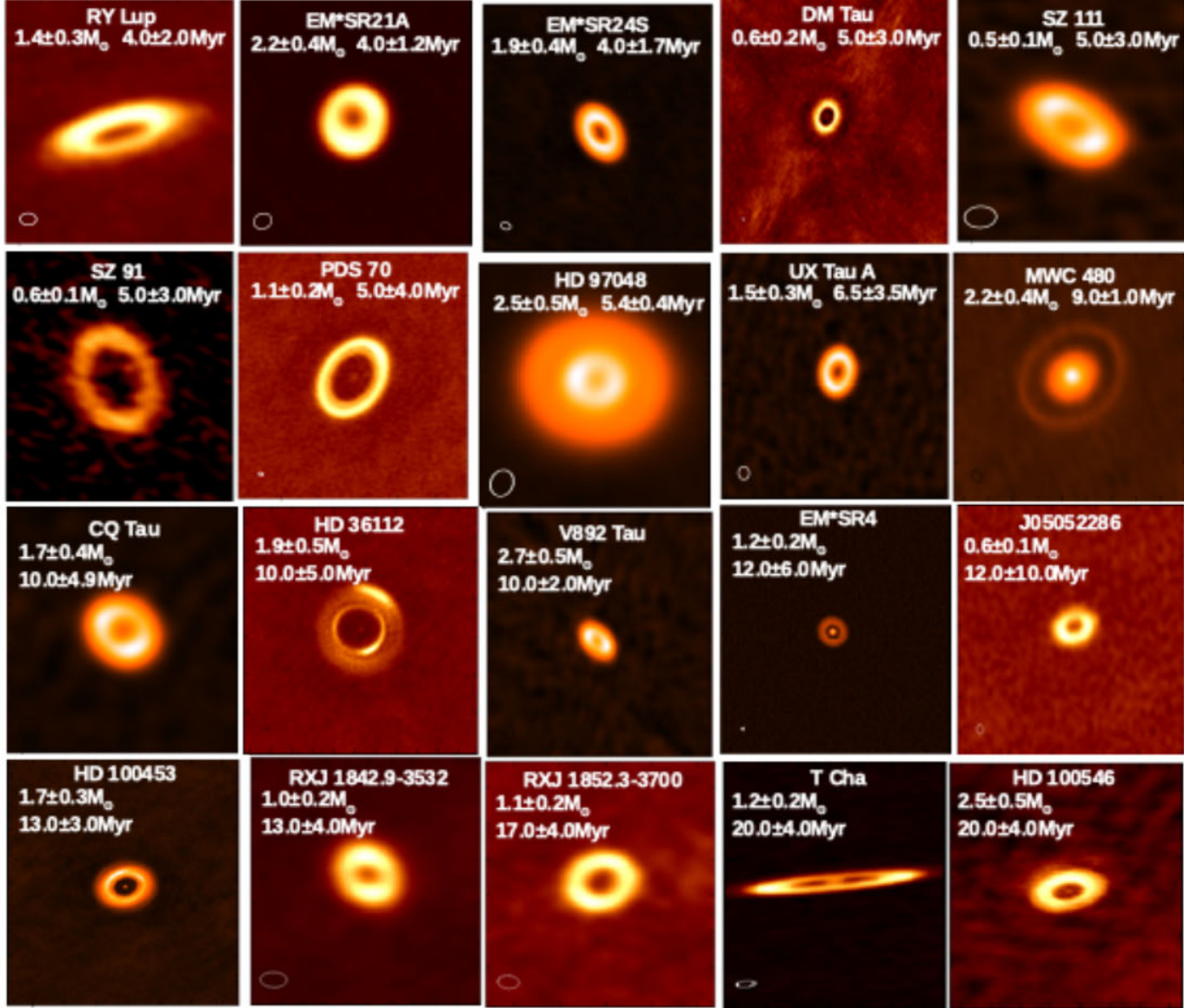

RXJ 1842.9-3532 $1.0 \pm 0.2 \mathrm{M}$ $13.0 \pm 4.0 \mathrm{Myr}$
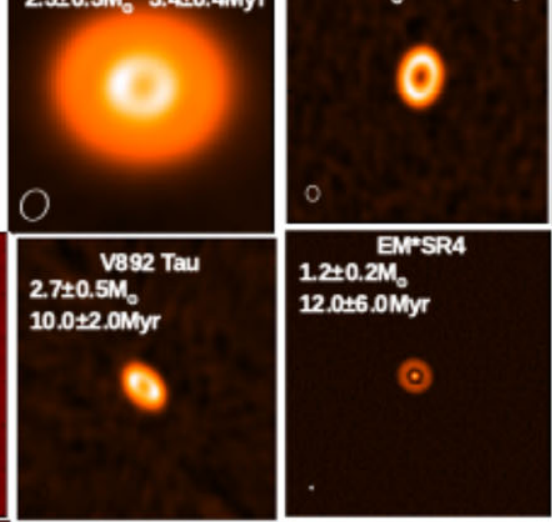

$2.2 \pm 0.4 \mathrm{M}_{0} 9.0 \pm 1.0 \mathrm{Myr}$

RXJ 1852.3-3700 $1.1 \pm 0.2 \mathrm{M}_{0}$

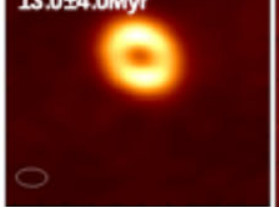
$17.0 \pm 4.0 \mathrm{Myr}$

e
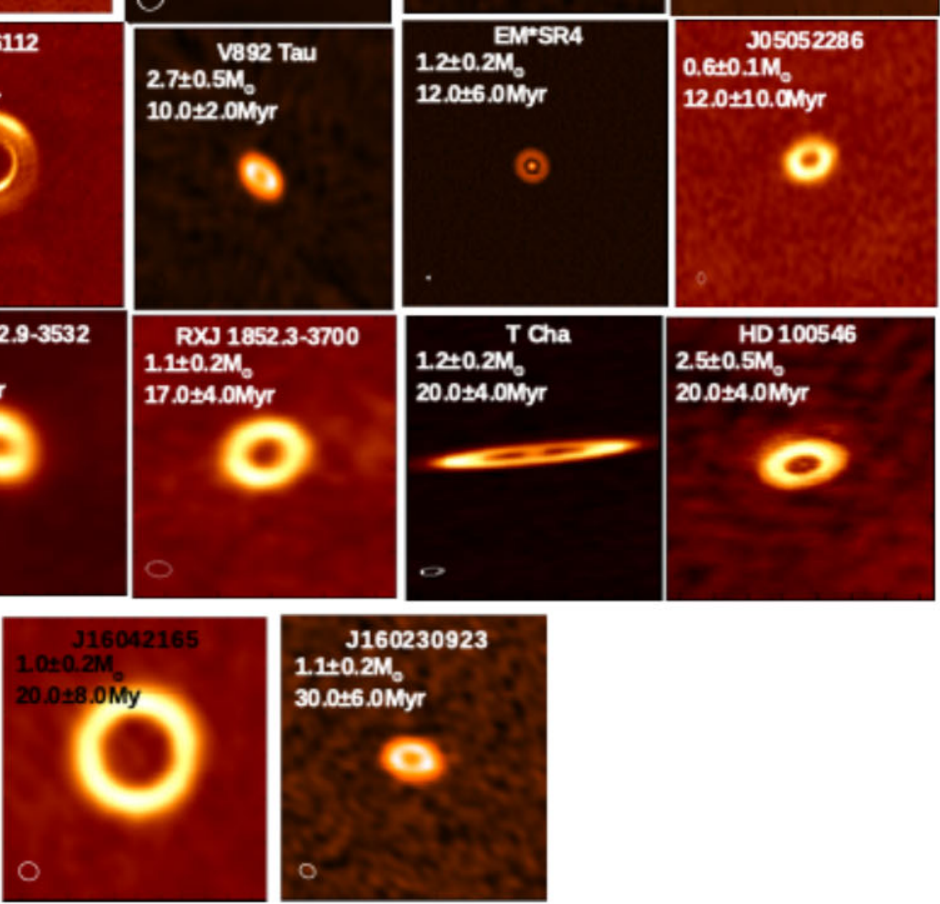

Figure C1. Images of the protoplanetary discs classified as Rims in this study. The discs are ordered by age with the top left (Elias 2-20) being the youngest and the lower right (J160230923) showing the oldest. The beam sizes used in each observation can be seen in the lower left of each image. All discs have been scaled to $500 \times 500$ au with the exception of the discs labelled with a $\dagger$ in the lower right. These discs have been scaled to $1000 \times 1000$ au. 


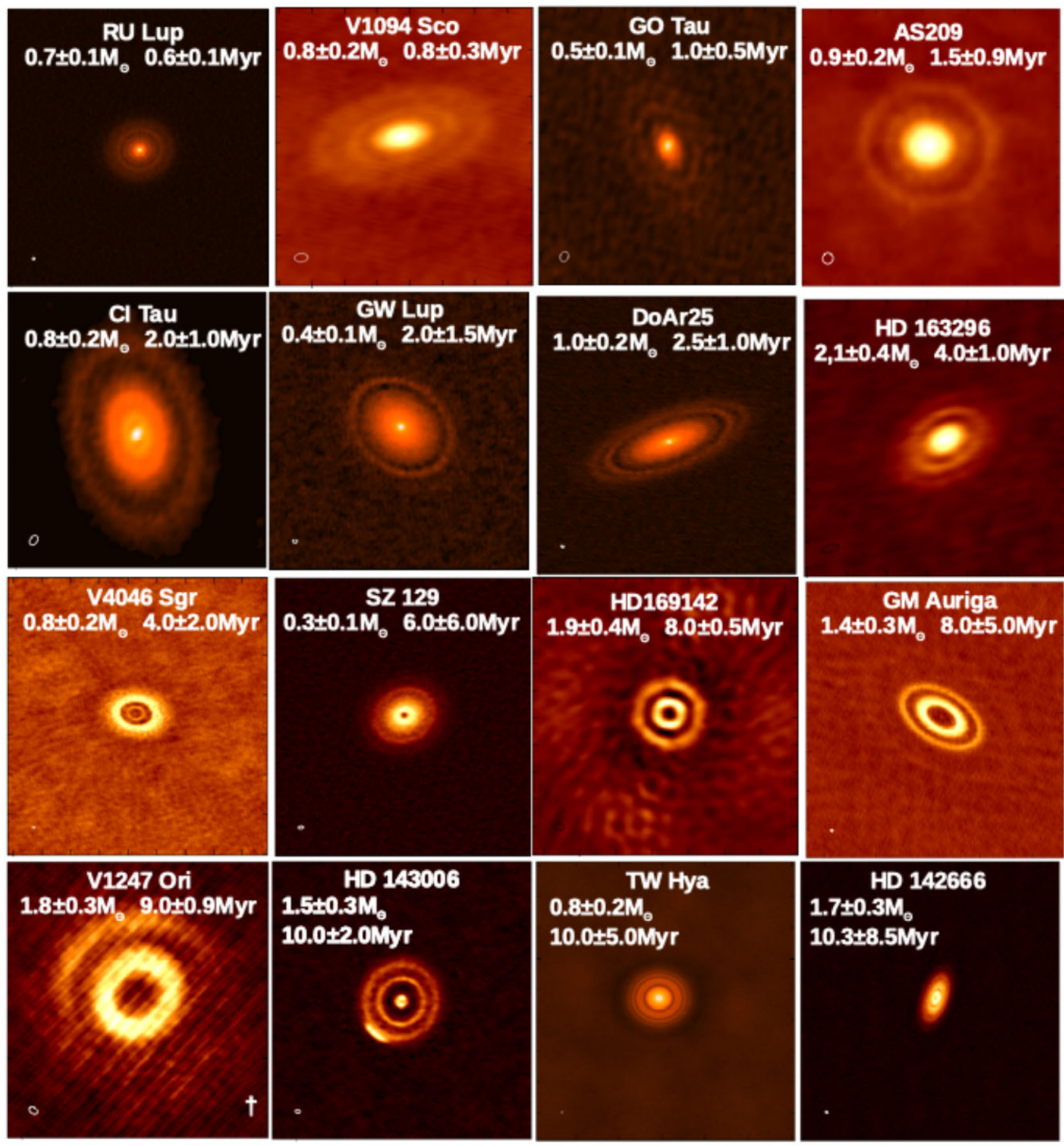

Figure C2. Images of the protoplanetary discs classified as Rings in this study. The discs are ordered by age with the top left (RU LUP) being the youngest and the lower right (HD 142666) showing the oldest. The beam sizes used in each observation can be seen in the lower left of each image. All discs have been scaled to $500 \times 500$ au with the exception of the discs labelled with a $\dagger$ in the lower right. These discs have been scaled to $1000 \times 1000$ au. 

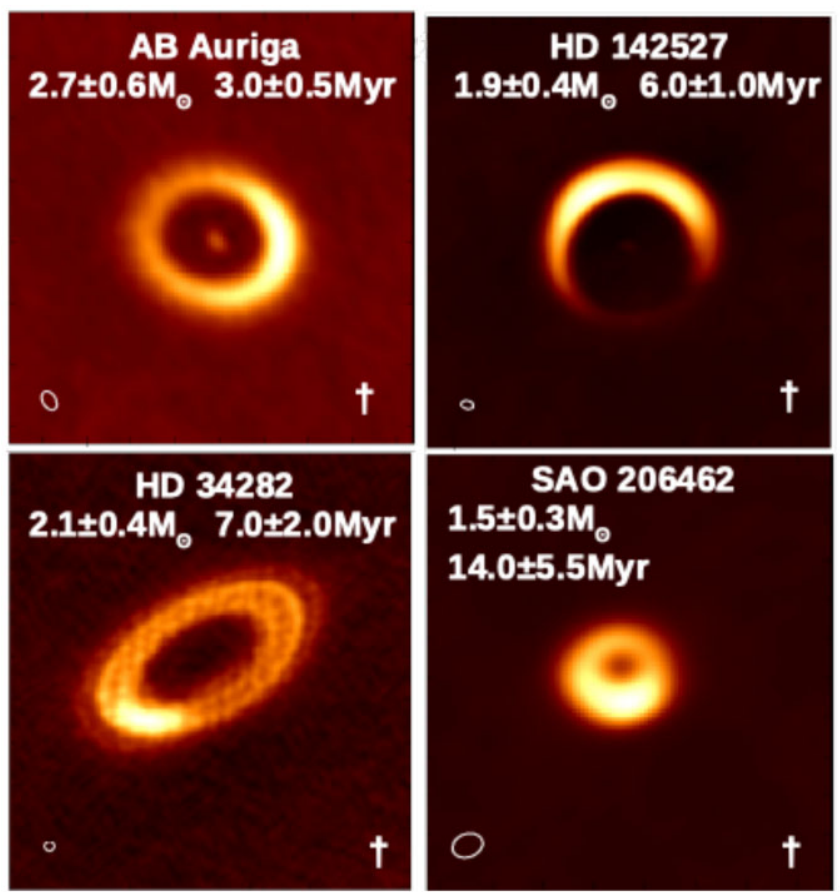

Figure C3. Images of the protoplanetary discs classified as Horseshoe in this study. The discs are ordered by age with the top left (AB Auriga) being the youngest and the lower right (HD100546) showing the oldest. The beam sizes used in each observation can be seen in the lower left of each image. The discs with the $\dagger$ in the lower right have been scaled to $1000 \times 1000 \mathrm{au}$.
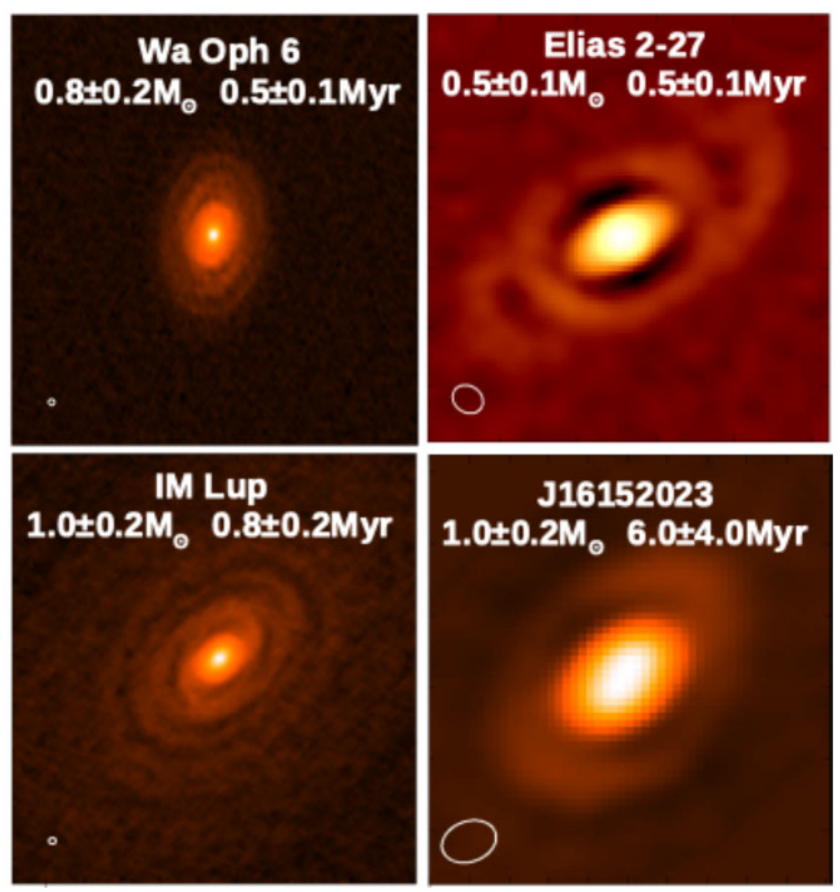

Figure C4. Images of the protoplanetary discs classified as Spiral in this study. The discs are ordered by age with the top left (Wa Oph 6) being the youngest and the lower right (J16152023) showing the oldest. The beam sizes used in each observation can be seen in the lower left of each image. 

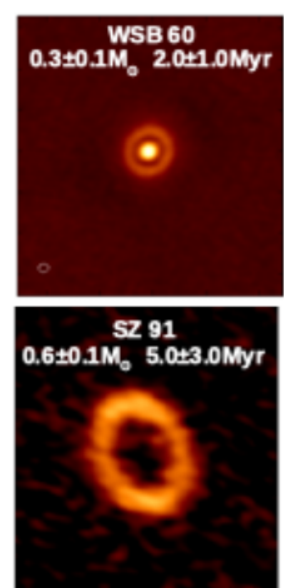

RXJ 1842.9-3532

$1.0 \pm 0.2 \mathrm{M}$

13.0.4.0Myr
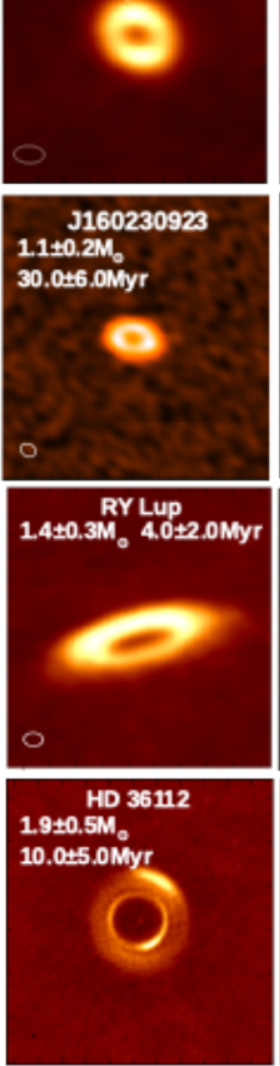

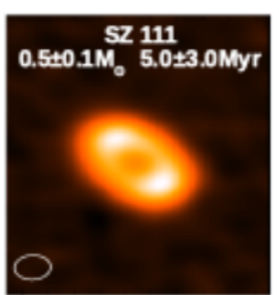

J05052286

$0.6 \pm 0.1 \mathrm{M}_{0}$

12.0ะ10.0Myr
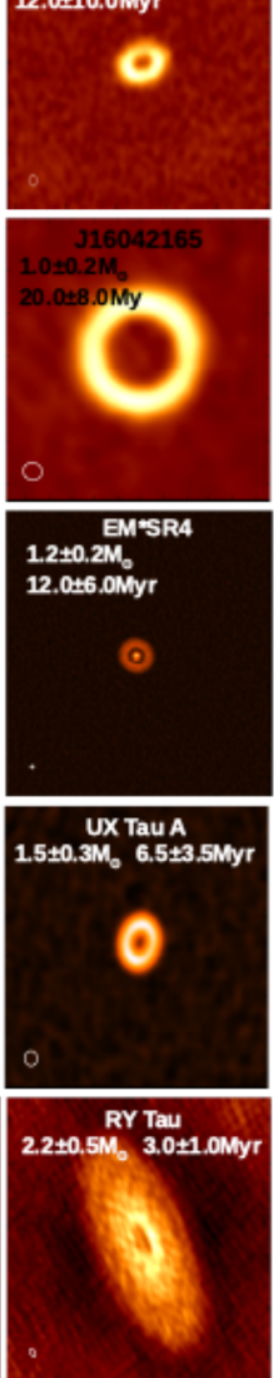

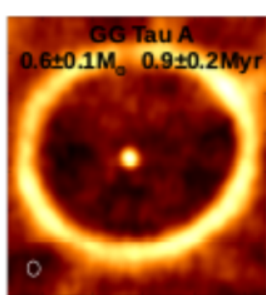

DS Tau

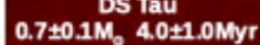

$0.7 \pm 0.1 \mathrm{M}, 4.0 \pm 1.0 \mathrm{Myr}$

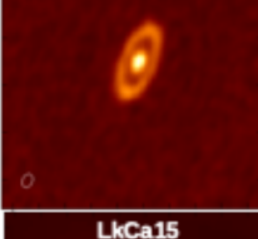

$1.1 \pm 0.2 \mathrm{M}_{0}, 3.0 \pm 2.0 \mathrm{Myr}$
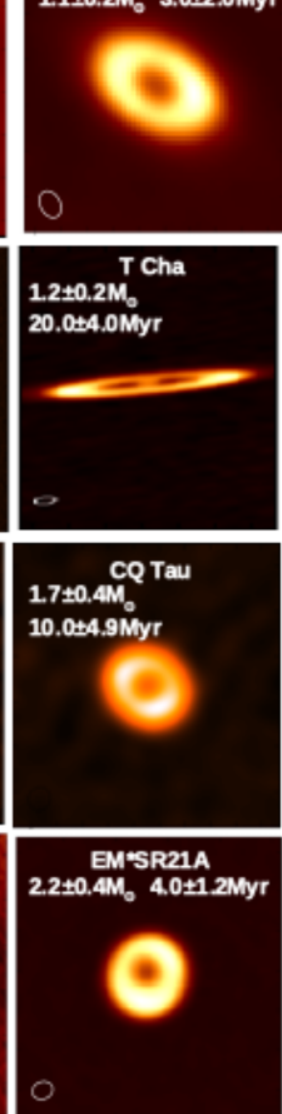

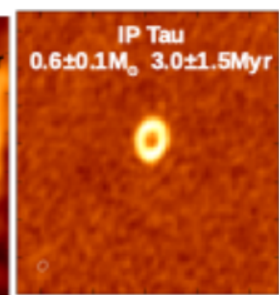

AATau

$0.8 \pm 0.2 \mathrm{M}_{0}, 1.5 \pm 0.8 \mathrm{Myr}$

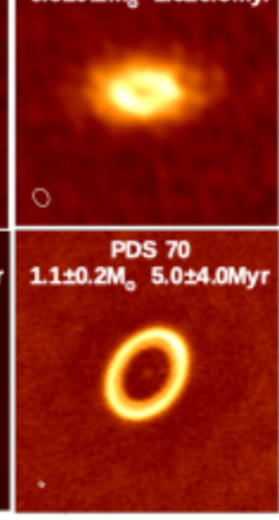

DoAr 44

$1.4 \pm 0.3 M_{0} 3.0 \pm 1.0 \mathrm{Myr}$

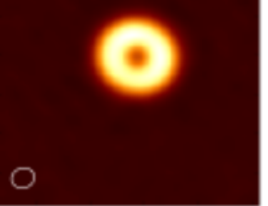

HD 100453

$1.7 \pm 0.3 \mathrm{M}_{0}$

13.0ะ3.0 Myr
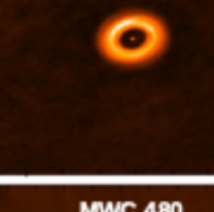

Mavc 480

$2.2 \pm 0.4 \mathrm{M}_{6} 9.0 \pm 1.0 \mathrm{Myr}$
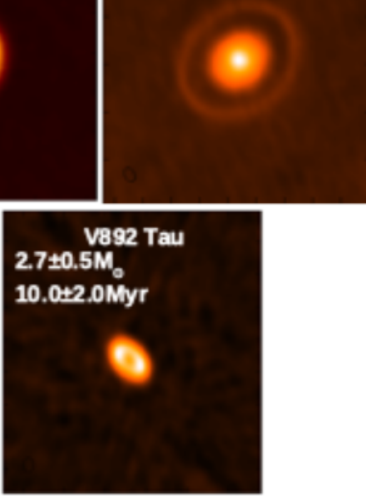

$2.7 \pm 0.5 \mathrm{M}$

$10.0+2.0 \mathrm{Mr}$
DM Tau

$0.6 \pm 0.2 \mathrm{M}_{6}, 5.0 \pm 3.0 \mathrm{Myr}$

0

PDS 99

$0.8 \pm 0.2 \mathrm{M}_{0}, 2.0 \pm 1.0 \mathrm{Myr}$

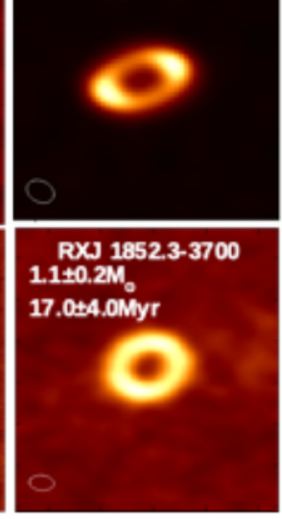

Cs Cha

$1.4 \pm 0.2 M_{6} 4.0 \pm 3.0 \mathrm{Myr}$

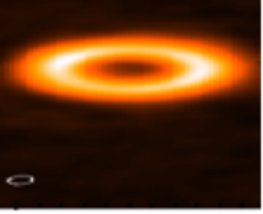

EM-SR24S

$1.9 \pm 0.4 M_{0} 4.0 \pm 1.7 \mathrm{Myr}$
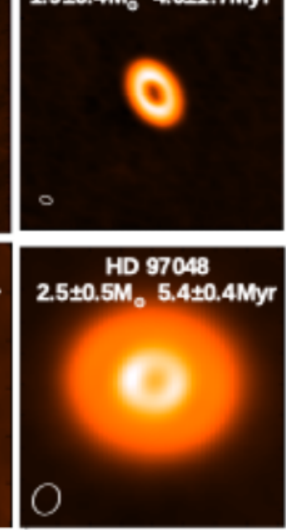

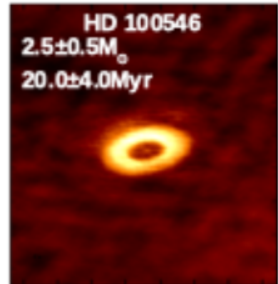

Figure C5. The Rim protoplanetary discs have been ordered according to stellar mass. WSB 60 has the lowest stellar mass of $M_{*}=0.3_{\odot}$, while RY Tau has a stellar mass of $M_{*} 3.0_{\odot}$. All discs have been scaled to $500 \times 500$ au. The discs with the $\dagger$ in the lower right have been scaled to $1000 \times 1000$ au. 

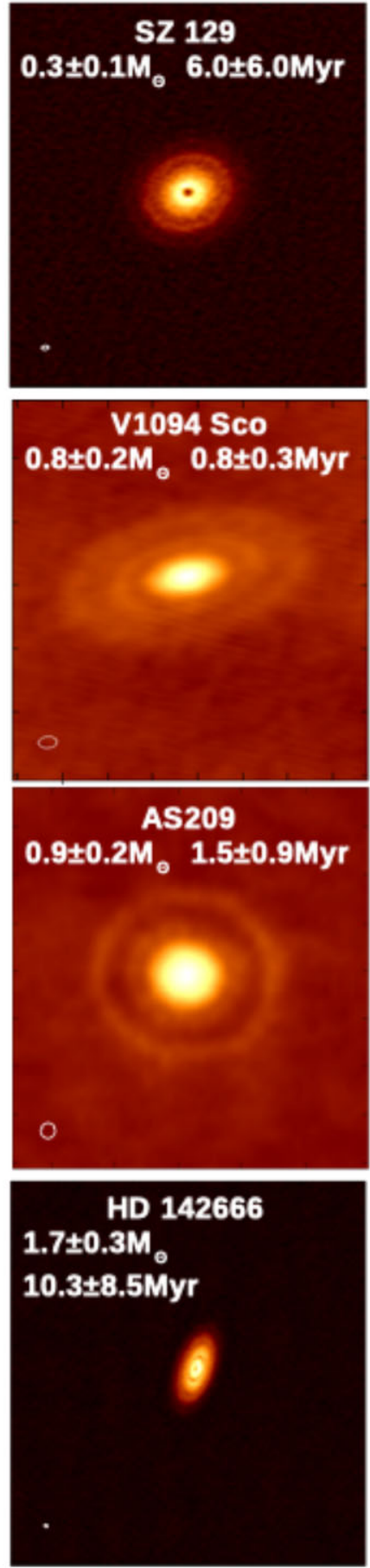
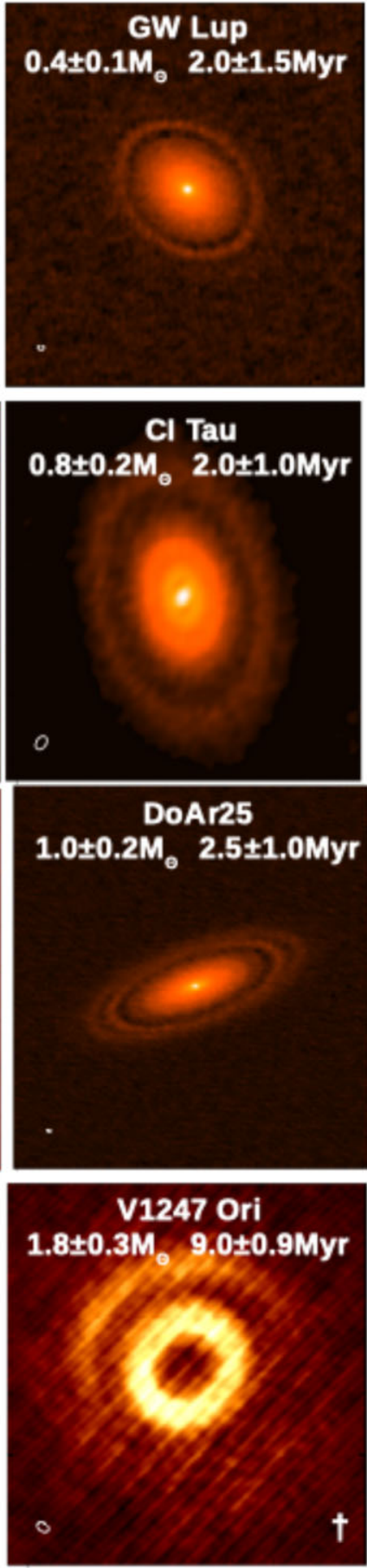

$\dagger$
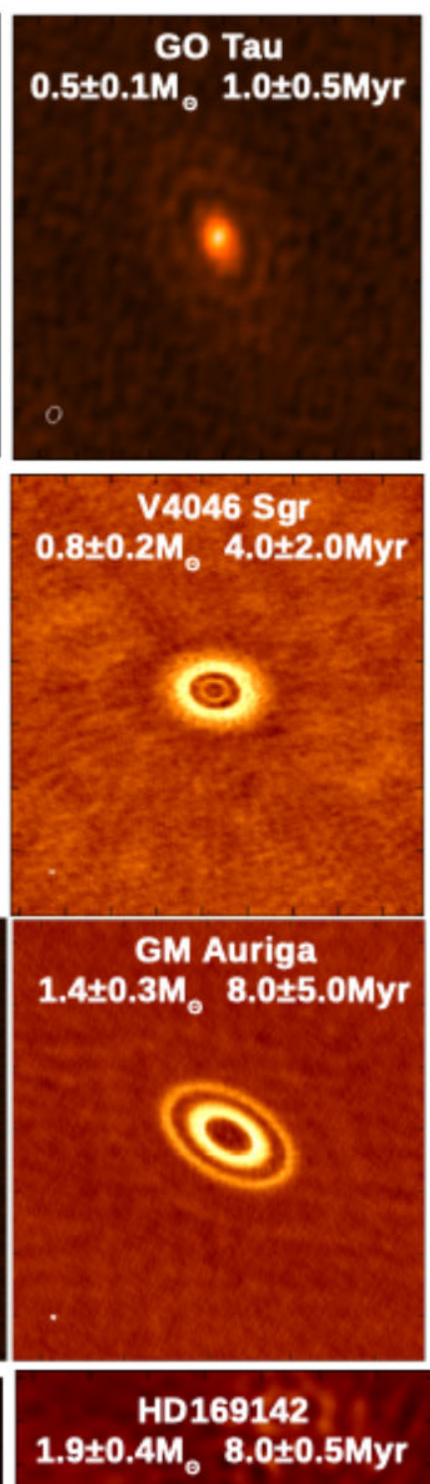

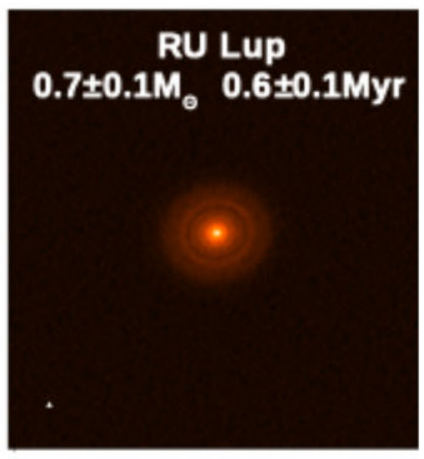

TW Hya
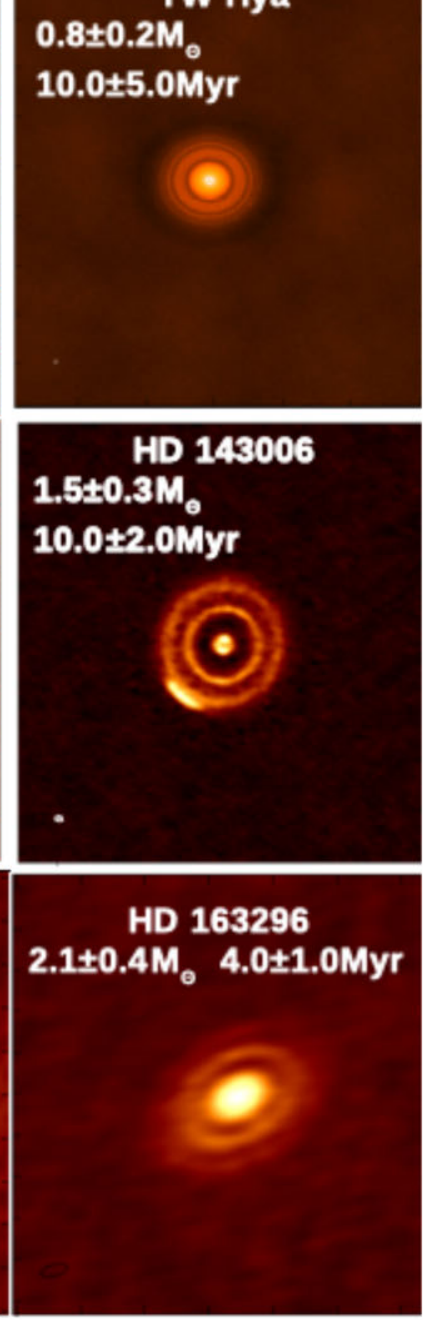

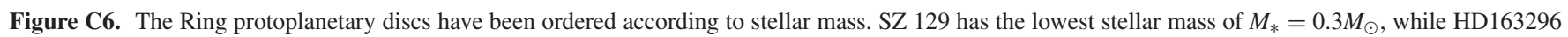
has a stellar mass of $M_{*}=2.1 \odot$. All discs have been scaled to $500 \times 500 \mathrm{au}$. The discs with the $\dagger$ in the lower right have been scaled to $1000 \times 1000$ au. 

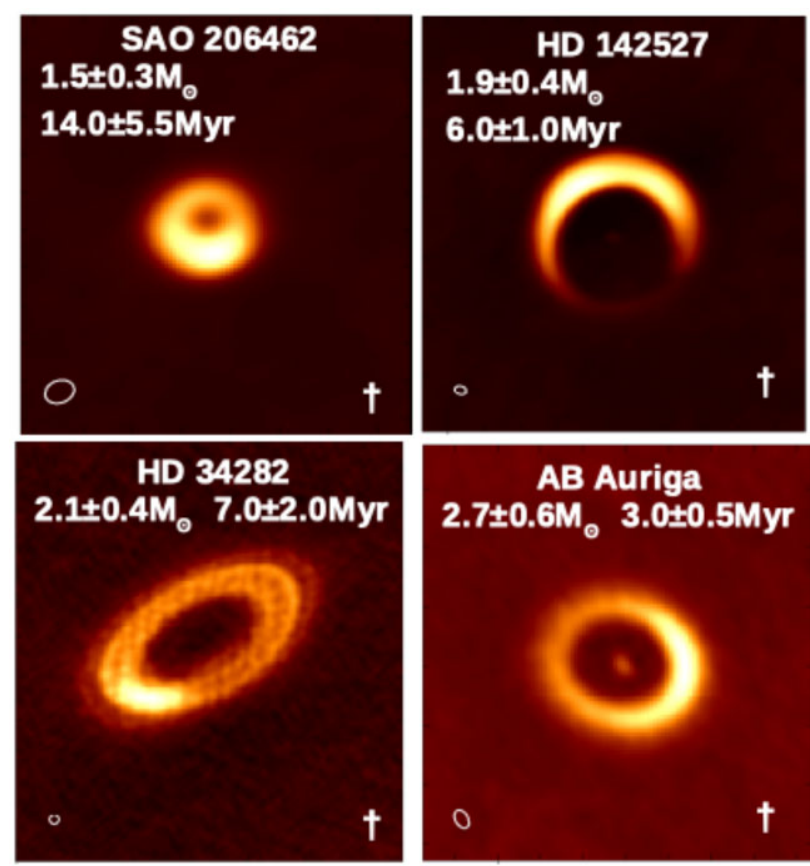

Figure C7. The Horseshoe protoplanetary discs have been ordered according to stellar mass. SAO206462 has the lowest stellar mass of $M_{*}=1.5 M_{\odot}$, while AB Auriga has a stellar mass of $M_{*}=2.7_{\odot}$. The discs with the $\dagger$ in the lower right have been scaled to $1000 \times 1000 \mathrm{au}$.
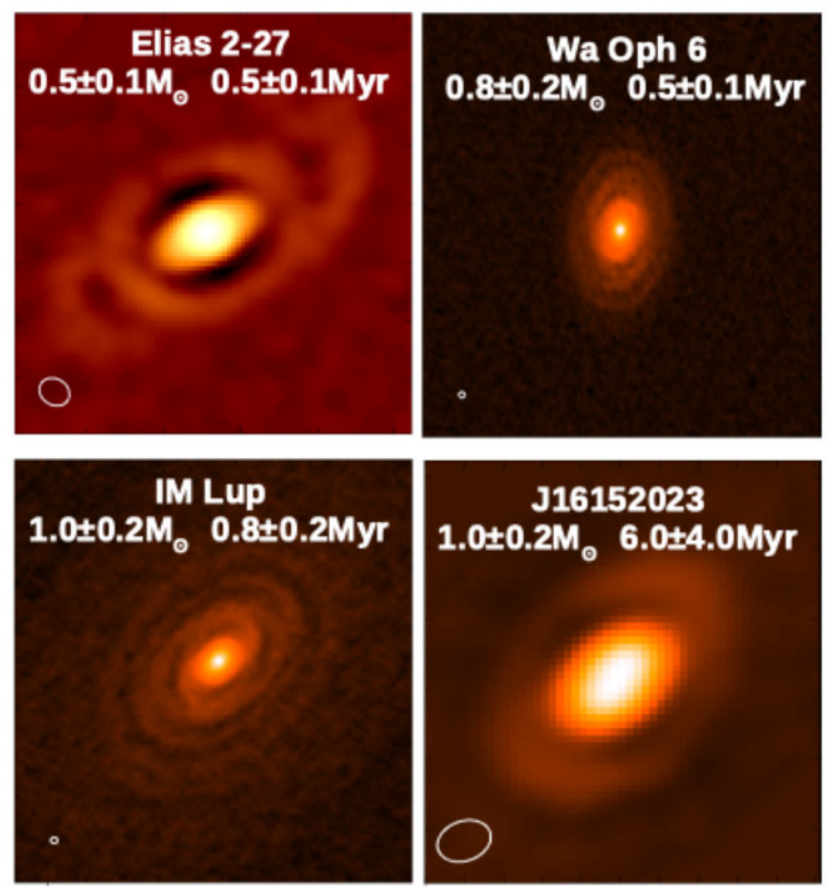

Figure C8. The Spiral protoplanetary discs have been ordered according to stellar mass. Elias 2-27 has the lowest stellar mass of $M_{*}=0.5 \mathrm{M}_{\odot}$, while $\mathrm{J} 16152023$ has a stellar mass of $M_{*}=1.0_{\odot}$. The discs have been scaled to $500 \times 500$ au.

This paper has been typeset from a $\mathrm{T}_{\mathrm{E}} \mathrm{X} / \mathrm{L} \mathrm{T} \mathrm{E} \mathrm{X}$ file prepared by the author. 